\title{
DERIVED TRACES OF SOERGEL CATEGORIES
}

\author{
EUGENE GORSKY, MATTHEW HOGANCAMP, AND PAUL WEDRICH
}

\begin{abstract}
We study two kinds of categorical traces of (monoidal) dg categories, with particular interest in categories of Soergel bimodules. First, we explicitly compute the usual Hochschild homology, or derived vertical trace, of the category of Soergel bimodules in arbitrary types. Secondly, we introduce the notion of derived horizontal trace of a monoidal dg category and compute the derived horizontal trace of Soergel bimodules in type $A$. As an application we obtain a derived annular Khovanov-Rozansky link invariant with an action of full twist insertion, and thus a categorification of the HOMFLY-PT skein module of the solid torus.
\end{abstract}

\section{CONTENTS}

1. Introduction 1

Acknowledgements $\quad 9$

Funding 9

2. Facts from homological algebra 9

3. Standard dg categorical constructions $\quad 15$

4. Homotopy idempotents and the Karoubi envelope 18

5. The bar complex for dg categories 30

6. The dg monoidal center and trace 39

7. Traces of the Soergel category 52

8. The Soergel category in type A 59

$\begin{array}{ll}\text { References } & 70\end{array}$

\section{INTRODUCTION}

1.1. Traces. Traces are ubiquitous in mathematics. If $A$ is an algebra over a field $\mathbb{k}$, its trace (or cocenter) is defined as the quotient $\mathrm{HH}_{0}(A)=A /[A, A]$. Given any finite-dimensional $A$-module $M$, the $\operatorname{trace}^{\operatorname{tr}_{M}}: A \rightarrow \mathbb{k}$ of the $A$-action on $M$ satisfies $\operatorname{tr}_{M}(x y)=\operatorname{tr}_{M}(y x)$ and hence factors through $A /[A, A]$. The projection $A \rightarrow A /[A, A]$ can thus be considered as a universal trace on $A$.

We are interested in traces for categories. If $\mathcal{C}$ is a $\mathbb{k}$-linear category, then its trace is a vector space over $\mathbb{k}$. If $\mathcal{C}$ is a monoidal category (or a 2-category) then its trace is a 1-category. Abstractly, the trace of an $n$-category satisfying certain assumptions is an $(n-1)$-category related to the factorization homology of the circle (see e.g. [AF19] for an introduction), but we will not employ this point of view in the present 
paper. Categorical traces have been studied in various settings, see for example Ocneanu [Ocn94], Evans-Kawahigashi [EK95], Walker [Wal], Ben-Zvi-Nadler [BN09], Ponto-Shulman [PS13], Beliakova-Lauda-Habiro-Živković [BHLv17], Hoyois-Scherotzke-Sibilla [HSS17], Beliakova-Putyra-Wehrli [BPW19].

In this paper we construct and study the derived traces of monoidal dg categories, with a view towards applications in higher representation theory and link homology.

Given a $\mathbb{k}$-linear dg category $\mathcal{C}$, one can define its Hochschild homology HH.(C) which is a vector space over $\mathbb{k}$. It is defined as homology of the explicitly defined cyclic bar complex which we review in Section 5.5. Keller proved [Kel06] that HH. (e) is a derived invariant of $\mathcal{C}$, for example, if $\mathcal{C}$ is the category of perfect complexes over an algebra $A$ then HH. (e) is isomorphic to the usual Hochschild homology of $A$. Note that $\mathrm{HH}_{0}(A)=A /[A, A]$ suggesting the interpretation of Hochschild homology as a derived (vertical) trace.

If $\mathrm{C}$ is a monoidal category (or a bicategory), then there is a richer notion of horizontal trace $\operatorname{Tr}_{0}(\mathcal{C})$ which is well-studied in various levels of generality. This is a category equipped with the "trace functor" $\operatorname{Tr}_{0}: \mathcal{C} \rightarrow \operatorname{Tr}_{0}(\mathcal{C})$. If $\mathcal{C}$ has left duals, then $\operatorname{Tr}_{0}$ is initial among all trace-like functors ${ }^{1} F: \mathcal{C} \rightarrow \mathcal{D}$, i.e. functors equipped with a natural transformation $F(X \otimes Y) \rightarrow F(Y \otimes X)$ respecting the tensor product in $\mathcal{C}$ (if $\mathcal{C}$ also has right duals, then the components of these natural transformations are necessarily isomorphisms). The horizontal trace is indeed a richer notion than the vertical trace, since the endomorphism algebra of $\operatorname{Tr}_{0}(\mathbb{1})$ in the horizontal trace naturally agrees with the vertical trace $\mathrm{HH}_{0}(\mathcal{C})$.

Example 1.1. The horizontal trace of the bicategory of tangles (where objects are finite sets of points in $I^{2}, 1$-morphisms are tangles in $I^{3}$, and 2-morphisms are tangle cobordisms in $I^{4}$ up to isotopy rel boundary) is the category of links in the thickened annulus (objects are links in $I^{2} \times S^{1}$, morphisms are link cobordisms in $I^{3} \times S^{1}$ up to isotopy rel boundary).

In this paper we define a derived version of the horizontal trace and prove the following:

Theorem 1.2. There is a natural $d g$ functor $\mathcal{C} \rightarrow \operatorname{Tr}(\mathcal{C})$, which is homotopy trace-like i.e. it is equipped with transformations $\operatorname{Tr}(X \otimes Y) \rightarrow \operatorname{Tr}(Y \otimes X)$ that are natural in $Y$ and natural up to coherent homotopy in $X$. The endomorphism algebra of $\operatorname{Tr}(\mathbb{1})$ is naturally isomorphic to $\mathrm{HH} .(\mathrm{C})$.

If $\mathcal{C}$ has left duals we expect that $\mathrm{Tr}$ is initial among all homotopy trace-like $\mathrm{dg}$ functors out of $\mathcal{C}$.

We also define the notion of $\mathrm{dg}$ Drinfeld center of $\mathcal{C}$ and prove that it acts on $\operatorname{Tr}(\mathcal{C})$; see section 6.6.

\footnotetext{
${ }^{1}$ Variations of trace-like functors are known under the names shadows [PS13], commutator functors [BFO09], categorical traces [HPT16], trace functors [Zhu18].
} 
It is desirable to consider the closure of $\operatorname{Tr}(\mathfrak{C})$ with respect to mapping cones and homotopy direct summands, which we denote by:

$$
\widetilde{\operatorname{Tr}}(\mathcal{C}):=\operatorname{Pretr}\left(\operatorname{Kar}^{d g}(\operatorname{Tr}(\mathcal{C}))\right),
$$

where $\operatorname{Kar}^{d g}$ and $\operatorname{Pretr}(-)$ respectively denote the homotopy idempotent completion and pretriangulated hull; see sections 3 and 4 for details.

1.2. Traces of Soergel bimodules. Next, we apply all the machinery of derived traces to categories of Soergel bimodules, starting with a computation of the derived vertical trace. Let $W$ be a Coxeter group with simple reflections $S \subset W$ and a realization $V$ over $\mathbb{C}$, and $\operatorname{SBim}(W)$ the associated monoidal category of Soergel bimodules [Soe92], which is a categorification of the Hecke algebra associated to the Coxeter system $(W, S)$. We set $R:=\operatorname{Sym}^{\bullet}\left(V^{*}\right)$, graded by placing $V^{*}$ in bidegree $(2,0)$ and $\Lambda:=\Lambda^{\bullet}\left(V^{*}\right)$, graded by placing $V^{*}$ in bidegree $(2,-1)$.

Theorem 1.3. We have an isomorphism of associative bigraded algebras

$$
\text { HH. }(\operatorname{SBim}(W)) \cong \mathrm{HH} .(R) \rtimes \mathbb{C}[W] .
$$

Remark 1.4. The Hochschild homology of the polynomial ring $R$ is canonically isomorphic to $R \otimes \Lambda$. After choosing a basis of $V$ we can identify HH. $(\operatorname{SBim}(W))$ with the algebra $\mathbb{C}\left[x_{1}, \ldots, x_{r}, \theta_{1}, \ldots, \theta_{r}\right] \rtimes \mathbb{C}[W]$ in which $x_{i}$ are even variables of degree $(2,0)$ and the $\theta_{i}$ are odd variables of degree $(2,-1)$, and $r=\operatorname{dim}(V)$.

Remark 1.5. In Theorem 1.3 the generators of the wreath product algebra on the right hand side are identified with the Hochschild cycles on the left hand side as follows. The generators of $R$ correspond to cycles $x \in \operatorname{Hom}(\mathbb{1}, \mathbb{1})$, the generators of $\Lambda$ correspond to the cycles $x\|\operatorname{Id}-\operatorname{Id}\| x \in \operatorname{Hom}(\mathbb{1}, \mathbb{1}) \otimes \operatorname{Hom}(\mathbb{1}, \mathbb{1})$ (see section 5 for notation conventions), and the elements $w \in W$ correspond to $(-1)^{\ell(w)} \operatorname{Id}_{\Delta_{w}} \in$ $\operatorname{Hom}\left(\Delta_{w}, \Delta_{w}\right)$ where $\Delta_{w}$ is the Rouquier complex corresponding to positive braid lift of $w$, and $\ell(w)$ is the length of $w$. See Theorem 7.6 for details.

Remark 1.6. In [EL16] the isomorphism $\mathrm{HH}_{0}(\operatorname{SBim}(W)) \cong R \rtimes \mathbb{C}[W]$ was proved by completely different methods, using cellularity of $\operatorname{SBim}(W)$.

Conjecture 1.7. $\mathrm{C}(\operatorname{SBim}(W))$ is formal as dg algebra, so higher $A_{\infty}$-operations on Hochschild homology vanish.

To support this conjecture, we prove a closely related Theorem 7.13 stating that $\operatorname{End}_{\operatorname{Tr}}(\operatorname{Tr}(K))$ is formal as a dg algebra, where $K$ is a certain "cube complex" built out of several copies of $\mathbb{1}$. Note that the conjecture describes formality of $\operatorname{End}_{\operatorname{Tr}}(\operatorname{Tr}(\mathbb{1})$ ).

After proving Theorem 1.2 we specialise to Soergel bimodules $\mathrm{SBim}_{n}$ for the symmetric group $S_{n}$, which feature in triply-graded Khovanov-Rozansky link homology [Kho07]. In [GW19] the first and third authors studied the category of annular webs and foams, which can be regarded as Karoubi completion of the horizontal trace $\operatorname{Tr}_{0}\left(\mathrm{SBim}_{n}\right)$. In particular, they proved that this Karoubi completion is generated by the direct summands of $\operatorname{Tr}_{0}\left(\mathbb{1}_{n}\right)$. Here we generalise this result to the derived horizontal trace using slightly different methods. 
Theorem 1.8. The dg functor $\operatorname{Hom}_{\operatorname{Tr}\left(\operatorname{SBim}_{n}\right)}\left(\operatorname{Tr}\left(\mathbb{1}_{n}\right),-\right)$ induces a quasi-equivalence relating $\widetilde{\operatorname{Tr}}\left(\operatorname{SBim}_{n}\right)$ to the category of perfect right $A_{\infty}$-modules over $\operatorname{End}\left(\operatorname{Tr}\left(\mathbb{1}_{n}\right)\right)$. In other words, we have a quasi-equivalence

$$
\begin{aligned}
\widetilde{\operatorname{Tr}}\left(\operatorname{SBim}_{n}\right) & \cong \operatorname{Perf}\left(\mathrm{HH}_{.}(R) \rtimes \mathbb{C}\left[S_{n}\right]\right) \\
& \cong \operatorname{Perf}\left(\mathbb{C}\left[x_{1}, \ldots, x_{n}, \theta_{1}, \ldots, \theta_{n}\right] \rtimes \mathbb{C}\left[S_{n}\right]\right),
\end{aligned}
$$

where $\operatorname{deg}\left(x_{i}\right)=(2,0)$ and $\operatorname{deg}\left(\theta_{i}\right)=(2,-1)$.

Note that projective modules over the algebra HH. $(R) \rtimes \mathbb{C}\left[S_{n}\right]$ are naturally indexed up to isomorphism by partitions of $n$, and under the equivalence of categories above every object of $\widetilde{\operatorname{Tr}}\left(\operatorname{SBim}_{n}\right)$ can be expressed as a twisted complex whose terms are (direct sums of shifts of) these projective modules. In other words, if $\mathbf{e}_{\lambda}$ is a projector in $\mathbb{C}\left[S_{n}\right]$ onto an irreducible representation $V_{\lambda}$, then we define the Schur object

$$
\mathbb{S}^{\lambda}:=\mathbf{e}_{\lambda} \operatorname{Tr}\left(\mathbb{1}_{n}\right)
$$

in $\widetilde{\operatorname{Tr}}\left(\operatorname{SBim}_{n}\right)$. A perfect $A_{\infty}$-module over HH. $(R) \rtimes \mathbb{C}\left[S_{n}\right]$ is then a twisted complex built out of $\mathbb{S}^{\lambda}$.

Remark 1.9. The type A Soergel bimodule categories taken together form the monoidal bicategory $\bigoplus_{n \geq 0} \mathrm{SBim}_{n}$, with the (new) tensor product $\otimes$ provided by induction $\mathrm{SBim}_{n} \times \mathrm{SBim}_{m} \rightarrow \mathrm{SBim}_{m+n}$. Its trace inherits the monoidal structure and is expected to admit a braiding that induces the $S_{n}$ action on $\operatorname{Tr}\left(\mathbb{1}_{1}\right)^{\unrhd n}=\operatorname{Tr}\left(\mathbb{1}_{n}\right)$. The Schur object $\mathbb{S}^{\lambda}$ is designed to be the evaluation of the $\lambda$-Schur functor on $\operatorname{Tr}\left(\mathbb{1}_{1}\right)$.

\subsection{Derived annular link invariants and categorification of the skein module of} the solid torus. The main motivation for this paper is to develop a framework for the categorification of the HOMFLY-PT skein module of the solid torus that is compatible with expectations from topological field theory, while at the same time allowing for explicit computations of the associated link invariant.

To describe this skein module, recall that the type $A_{n-1}$ Hecke algebra $H_{n}$ can be described as the linear span of braids on $n$ strands modulo skein relations and isotopies. The multiplication in $H_{n}$ is inherited from stacking braids, and the unit is represented by identity braid.

Similarly, the (positive half of the) skein module of the annulus $\operatorname{Sk}_{n}^{+}(\mathbb{A})$ is defined as the linear span of annular braid closures modulo skein relations and isotopies. It is easy to see from the definition that $\operatorname{Sk}_{n}^{+}(\mathbb{A})$ is isomorphic to the cocenter of the Hecke algebra:

$$
\mathrm{Sk}_{n}^{+}(\mathbb{A}) \simeq \frac{H_{n}}{\left[H_{n}, H_{n}\right]}
$$

Any trace function $f$ on $H_{n}$, i.e. a linear function satisfying $f(x y)=f(y x)$, naturally factors through the cocenter, and hence can be viewed as a function on $\operatorname{Sk}_{n}^{+}(\mathbb{A})$.

Let $\Lambda_{q}$ denote the ring of symmetric functions in infinitely many variables over $\mathbb{C}(q)$, and let $\Lambda_{q}^{(n)}$ denote the subspace of degree $n$ symmetric functions. The skein module of the annulus enjoys the following properties: 
(a) $\mathrm{Sk}_{n}^{+}(\mathbb{A})$ is isomorphic to $\Lambda_{q}^{(n)}$. It has a basis of Schur functions $s_{\lambda}$ labeled by partitions $\lambda$ with $n$ boxes.

(b) The HOMFLY-PT invariant of links yields a trace on $H_{n} \rightarrow \mathbb{C}(q)\left[a, a^{-1}\right]$ (called the Jones-Ocneanu trace), and can be computed by projecting $H_{n}$ to $\mathrm{Sk}_{n}^{+}(\mathbb{A}) \cong$ $\Lambda_{q}^{(n)}$ and applying a certain algebra map $\Lambda_{q} \rightarrow \mathbb{C}(q)\left[a, a^{-1}\right]$.

(c) The center of $H_{n}$ naturally acts on its cocenter. On the level of annular link diagrams this corresponds to cutting open the annular link diagram and inserting a central element before closing it again. In particular, the full twist is central in the braid group and hence acts on $\operatorname{Sk}_{n}^{+}(\mathbb{A})$.

Links in $S^{1} \times D^{2}$ may be studied by means of their diagrams in $\mathbb{A}$, after choosing a homeomorphism $S^{1} \times D^{2} \cong \mathbb{A} \times I$. Such a homeomorphism will be referred to as an $I$ bundle structure on $S^{1} \times D^{2}$. A framing is a choice of $I$-bundle structure up to isotopy. A choice of $I$-bundle structure gives us a well-defined link diagram associated to generic links $L$, whereas a choice of framing determines a diagram only up to Reidemeister moves. Two different framings are related by some number of twists, which on the level of link diagrams corresponds to the insertion of some power of the full-twist braid, as in (c) above.

For this reason, if one is interested in (say, the positive half) of the skein module of a 3-manifold $Y$ which is homeomorphic to $S^{1} \times D^{2}$ (but with no preferred homeomorphism) then it is necessary to understand not just the skein module $\operatorname{Sk}_{n}^{+}(\mathbb{A})$, but also the automorphism of full twist insertion.

The categorification of the skein module proceeds in several steps. First, the Hecke algebra $H_{n}$ is categorified by the monoidal category $\mathrm{SBim}_{n}$ of Soergel bimodules in type $A_{n-1}$, or by a closely related monoidal category of webs and foams defined by Queffelec-Rose [QR16, Remark 3.24], see [MV10], [Wed19, Remark 3.3], and [RW16, Section 4.4] for the connection. In the second step one must categorify the cocenter of $H_{n}$. Traditionally (see Queffelec-Rose [QR18], Beliakova-Putyra-Wehrli [BPW19], and Queffelec-Rose-Sartori [QRS18]) this is done using the underived horizontal trace $\operatorname{Tr}_{0}$. This underived horizontal trace is satisfactory for many purposes. For instance Queffelec-Rose-Sartori proved in [QRS18] that the triply-graded KhovanovRozansky homology KhR [KR08, Kho07] factors through the underived horizontal trace, which gives a categorification of (b) above. Additionally, in [GW19] the first and third authors connected the annular Khovanov-Rozansky invariant of QueffelecRose [QR18] to the underived horizontal trace of type A Soergel bimodules $\operatorname{Tr}_{0}\left(\operatorname{SBim}_{n}\right)$ and showed that a categorification of (a) holds upon Karoubi completion.

Note that $\operatorname{Tr}_{0}\left(\operatorname{SBim}_{n}\right)$ indeed categorifies $\Lambda_{q}^{(n)}$, as it is generated by objects $\mathbb{S}^{\lambda}$ as in (2), which correspond to Schur functions $s_{\lambda}$. So the Grothendieck group of $\operatorname{Tr}_{0}\left(\operatorname{SBim}_{n}\right)$ is naturally isomorphic to $\Lambda_{q}^{(n)}$.

For a categorification of the skein module of the solid torus, we also need automorphisms of the target category which realise changes in $I$-bundle structure, 
as in property (c) above. The following example shows that the ordinary annular Khovanov-Rozansky link invariant, which is constructed using the underived horizontal trace, does not enjoy this property.

Example 1.10. The annular Khovanov-Rozansky invariant of a 2-component unlink decomposes into two non-trivial direct summands $\operatorname{AKhR}\left(\mathbb{1}_{2}\right) \cong S^{2} \oplus \wedge^{2}$. Here $S^{2}$ and $\wedge^{2}$ denote the Schur objects $\mathbb{S}^{(2)}$ and $\mathbb{S}^{(1,1)}$ as in (2). Twisting the $I$-bundle structure turns the unlink into an annular Hopf link-the braid closure of the full twist on two strands-whose invariant is a chain complex

$$
\operatorname{AKhR}\left(\mathrm{FT}_{2}\right) \simeq \underline{\wedge^{2}(-2) \oplus \wedge^{2}} \oplus\left(\underline{0} \longrightarrow \wedge^{2} \longrightarrow x_{1}-x_{2} \longrightarrow S^{2}(2)\right) .
$$

which decomposes into three non-trivial direct summands. Changes in $I$-bundle structure do not induce isomorphisms on annular Khovanov-Rozansky invariant. Here we have used the version of AKhR defined in [GW19], but the same argument applies to all other constructions employing the horizontal trace.

To remedy this issue, we use the derived horizontal trace.

Definition 1.11. We define the derived annular Khovanov-Rozansky invariant of a braid word $\beta$ on $n$ strands, denoted $\operatorname{AKhR}_{\mathrm{dg}}(\beta)$, to be the derived horizontal trace class of the Rouquier complex of $\beta$ in $\operatorname{Tr}\left(\operatorname{SBim}_{n}\right)$.

By Theorem 1.8, this invariant can be considered as taking values in perfect $A_{\infty}$ modules over the $A_{\infty}$ algebra $\mathbb{C}\left[x_{1}, \ldots, x_{n}, \theta_{1}, \ldots, \theta_{n}\right] \rtimes \mathbb{C}\left[S_{n}\right]$, where $S_{n}$ is supported in cohomological degree zero and the variables $x_{i}$ and $\theta_{i}$ have cohomological degree 0 and -1 respectively. Unlike for AKhR, changes in $I$-bundle structure induce automorphisms on $\mathrm{AKhR}_{\mathrm{dg}}$. These arise naturally through the action of the derived central Rouquier complex of the full twist braid on the derived horizontal trace.

Example 1.12. The derived annular Khovanov-Rozansky invariant of the full twist on two strands is a twisted complex

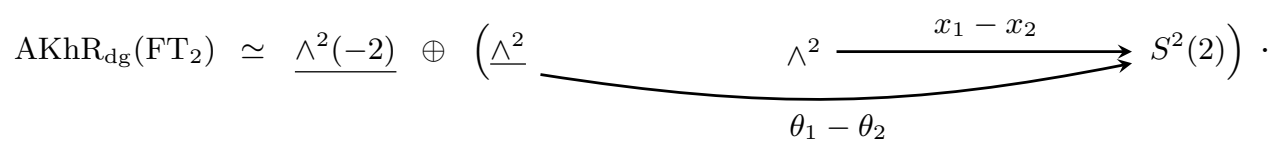

Like $\operatorname{AKhR}_{\mathrm{dg}}\left(\mathbb{1}_{2}\right) \cong S^{2} \oplus \wedge^{2}$, this has two indecomposable direct summands. The action of the derived central full twist on $A K h R_{\text {dg }}$ sends $\wedge^{2}$ to $\wedge^{2}(-2)$ and $S^{2}$ to the twisted complex shown as the second direct summand above.

Remark 1.13. The indecomposable summands of $\mathrm{AKhR}_{\mathrm{dg}}\left(\mathrm{FT}_{n}\right)$ are nothing but the images of indecomposable summands of $\operatorname{AKhR}_{\mathrm{dg}}\left(\mathbb{1}_{n}\right)$ (that is, $\mathbb{S}^{\lambda}$ for partitions $\lambda$ of $n$ ) under the action of the full twist. Following the conjectures of the first author, Negut and Rasmussen [GNR16], we expect the action of the full twist to be closely related to the action of Bergeron-Garsia operator $\nabla$ originating in the theory of Macdonald polynomials [BGHT99]. 
In future work, we will use $\mathrm{AKhR}_{\mathrm{dg}}$ to study cabling operations for KhovanovRozansky link homologies. We also anticipate that the technology of derived traces will be useful in the program to categorify skein algebras, see e.g. [QW18], and for explicit computations of the vector space-valued 4-manifold invariants derived from Khovanov-Rozansky link homology [MWW19].

1.4. Comparison with character sheaves. In this section we briefly compare our results to the theory of character sheaves. Let $G$ be a semi-simple split algebraic group with a Borel subgroup $B$ and Weyl group $W$. Recall that a geometric categorification of the Hecke algebra for $W$ is given by the category of $B$-equivariant constructible sheaves on the flag variety $G / B$, or, equivalently, $B$-biequivariant sheaves on $G$. Other, very similar versions of the Hecke category include $B$-equivariant $D$-modules on $G / B$, or Harish-Chandra bimodules. By the work of Soergel the geometric Hecke category is closely related to the category of Soergel bimodules. The polynomial ring $\mathrm{R}$ corresponds to the B-equivariant cohomology of a point. For more details, see [Soe90].

In [BZFN10, BN09] both the trace and the center of the geometric Hecke category were identified with the category of Lusztig's character sheaves [Lus84]. In particular, the object $\operatorname{Tr}(\mathbb{1})$ which plays a prominent role in the paper corresponds to the socalled Springer sheaf. The derived endomorphism algebra of the Springer sheaf is known (in particular, it is isomorphic to $\mathbb{C}[W]$ in degree zero), and the formality result similar to Theorem 1.3 was proved by Rider [Rid13]. It is important to mention that the results of [Rid13] hold in the category of mixed perverse sheaves which is equipped with an additional grading, which is analogous to our $q$-grading. See also [PVdB19, eq. 0.0.4] and [Li] for related results. On the other hand, in Theorem 1.8 we get wo sets of variables $\theta_{i}, x_{i}$ while the other references have only one set of variables.

Finally, it is known [Lus84, RR16] that in type A the summands of the Springer sheaf generate the category of character sheaves, while this is not the case in other types.

Note that we do not claim any results about the Drinfeld center of $\mathrm{SBim}_{n}$ or the corresponding category of complexes, but plan to compute it in the future work.

1.5. Comparison with Hilbert scheme of points. In [GNR16] the first author, Negut and Rasmussen proposed a set of conjectures relating the category of Soergel bimodules to the Hilbert scheme of points on the plane $\operatorname{Hilb}^{n}\left(\mathbb{C}^{2}\right)$. In particular, they conjectured that both the trace and the center of $\mathcal{K}^{b}\left(\mathrm{SBim}_{n}\right)$ are closely related to the derived category of coherent sheaves on $\operatorname{Hilb}^{n}\left(\mathbb{C}^{2}\right)$. We plan to work out the precise connection between this work and [GNR16] in the future, and only comment on one remarkable formal similarity.

Haiman constructed in [Hai01] a rank $n$ ! vector bundle $\mathcal{P}$ on $\operatorname{Hilb}^{n}\left(\mathbb{C}^{2}\right)$ called the Procesi bundle. Its endomorphism algebra has the form

$$
\operatorname{Hom}(\mathcal{P}, \mathcal{P})=\mathbb{C}\left[x_{1}, \ldots, x_{n}, y_{1}, \ldots, y_{n}\right] \rtimes \mathbb{C}\left[S_{n}\right], \operatorname{Ext}^{i}(\mathcal{P}, \mathcal{P})=0, i>0 .
$$


It is known that the direct summands of $\mathcal{P}$ generate the derived category of $\operatorname{Hilb}^{n}\left(\mathbb{C}^{2}\right)$, and hence the functor

$$
\operatorname{RHom}(\mathcal{P},-): D^{b}\left(\operatorname{Hilb}^{n}\left(\mathbb{C}^{2}\right)\right) \rightarrow D^{b}\left(\mathbb{C}\left[x_{1}, \ldots, x_{n}, y_{1}, \ldots, y_{n}\right] \rtimes \mathbb{C}\left[S_{n}\right] \text {-mod }\right)
$$

is an equivalence [BKR01]. The equation (3) is very similar to the endomorphism algebra of $\operatorname{Tr}\left(\mathbb{1}_{n}\right)$ appearing in Theorem 1.3 , but the odd variables $\theta_{i}$ of degree $(2,-1)$ are replaced by the even variables $y_{i}$ of degree $(-2,2)$. It is likely that the $\mathrm{dg}$ enhancement of $D^{b}\left(\mathrm{Hilb}^{n}\left(\mathbb{C}^{2}\right)\right)$ is related to the horizontal trace of $\mathrm{SBim}_{n}$ by some kind of Koszul duality.

1.6. Organization of the paper. In section 2 we set up notation and conventions for differential graded $(\mathrm{dg})$ categories and functors between them. Throughout the paper we chose to avoid any discussion of $A_{\infty}$-categories and $A_{\infty}$-functors, so we use the formalism of quasi-functors instead (see subsection 2.3). We also review the notion of formality for $\mathrm{dg}$ algebras and its relation to Massey products, see subsection 2.5.

In section 3 and 4 we discuss various notions of completion of dg categories with respect to direct sums, cones and homotopy idempotents. In particular, we define the pretriangulated hull and the dg Karoubi completion for an abstract dg category. This material is quite standard, and can be found, for example, in Seidel's book [Sei08], which we more or less follow; however, we decided to present it as concretely as possible for the readers' convenience. In particular, we avoid Yoneda embeddings altogether and explicitly construct $A_{\infty}$ lifts of homotopy idempotents (Proposition 4.15) which allows us to give a dg model for the Karoubi completion.

In section 5 we define and study the 2-sided bar complex of a dg category, its cyclic version and Hochschild homology of a dg category. In subsection 5.5 we compare the full Hochschild homology of the dg category with its vertical trace. The main result of this section is Theorem 5.19 where we prove that if a $\mathrm{dg}$ category admits a semiorthogonal decomposition then its cyclic bar complex retracts onto the direct sum of cyclic bar complexes for summands. This is a dg version of a result of Kuznetsov [Kuz09] on additivity of Hochschild homology in semiorthogonal decompositions.

The next section 6 is the technical core of the paper. We define and study the derived Drinfeld center and derived horizontal trace for monoidal dg categories. We prove Theorem 1.2 on the universal trace functor $\operatorname{Tr}: \mathcal{C} \rightarrow \operatorname{Tr}(\mathcal{C})$ and its properties, and also define an action of the derived center on the derived trace. Note that $\operatorname{Tr}(\mathcal{C})$ is usually not pretriangulated or idempotent complete, but the results of section 5 allow us to consider the corresponding completions.

In section 7 we apply this machinery to the monoidal category of Soergel bimodules and prove Theorem 1.3.

In section 8 we prove Theorem 1.8 and describe an explicit "annular simplification" algorithm which allows us to identify the trace of any type A Soergel bimodule with a homotopy summand in the direct sum of several copies of $\operatorname{Tr}(\mathbb{1})$. We also discuss the connections of the derived trace with annular Khovanov-Rozansky invariants and the work of the first and third author [GW19]. In particular, we construct a 
"forgetful functor" from the derived to the "underived" horizontal trace, and show that Khovanov-Rozansky homology of a braid closure factors through it, see Theorem 8.24 .

\section{ACKNOWLEDGEMENTS}

The authors would like to thank Anna Beliakova, Roman Bezrukavnikov, Tudor Dimofte, Ben Elias, Mikhail Gorsky, Tina Kanstrup, Bernhard Keller, Oscar Kivinen, Andrei Negut, Alexei Oblomkov, Jacob Rasmussen, David Reutter, Raphaël Rouquier, Lev Rozansky, Sarah Scherotzke, Catharina Stroppel, Kostya Tolmachov and Geordie Williamson for many useful discussions.

Special thanks to Kostya Tolmachov who found a gap in a proof of Conjecture 1.7 that was claimed in the first version of this paper.

This work also profited from discussions during the AIM workshop "Categorified Hecke algebras, link homology, and Hilbert schemes", the ESI Workshop "Categorification in quantum topology and beyond", the workshop "Hidden Algebraic Structures in Topology" at Caltech, the workshop "Hilbert schemes, categorification and combinatorics" at UC Davis, and the MSRI programs "Quantum Symmetries" and "Higher Categories and Categorification". We thank the host institutions and sponsors of these activities for their support.

\section{FUNDING}

Section 8 was elaborated by E.G. with the support by the Grant 16-11-10018 of the Russian Science Foundation. The work of E.G. in the other Sections was partially supported by NSF grants DMS-1700814 and DMS-1760329.

M. H. was partially supported by the NSF grant DMS-1702274.

P.W. was partially supported by the Australian Research Council grants 'Braid groups and higher representation theory' DP140103821 and 'Low dimensional categories' DP160103479 while at the Australian National University during early stages of this project.

\section{FACTS FROM HOMOLOGICAL ALGEBRA}

This section serves to recall important notions from homological algebra and to fix notation and conventions.

2.1. Complexes. Let $\mathbb{k}$ be a commutative ring. The category of complexes of $\mathbb{k}$ modules will be denoted $\mathrm{Ch}(\mathbb{k})$. Its objects are complexes of $\mathbb{k}$-modules, also called $d g \mathbb{k}$-modules, and we will use the cohomological convention for differentials:

$$
\ldots \stackrel{d}{\rightarrow} X^{k} \stackrel{d}{\rightarrow} X^{k+1} \stackrel{d}{\rightarrow} \ldots
$$


In particular, the differentials are considered to be of cohomological degree 1 . The morphism spaces between objects $X$ and $Y$ in $\mathrm{Ch}(\mathbb{k})$ are the complexes with

$$
\operatorname{Hom}_{\mathrm{Ch}(\mathbb{k})}^{k}(X, Y)=\prod_{i \in \mathbb{Z}} \operatorname{Hom}_{\mathbb{k}}\left(X^{i}, Y^{i+k}\right), \quad d_{\operatorname{Hom}_{\mathrm{Ch}(\mathbb{k})}(X, Y)}(f):=d_{Y} \circ f-(-1)^{|f|} f \circ d_{X}
$$

where $|f|$ denotes the cohomological degree of $f$. The full subcategory of bounded complexes will be denoted $\mathrm{Ch}^{b}(\mathbb{k})$. The categories $\mathrm{Ch}(\mathbb{k})$ and $\mathrm{Ch}^{b}(\mathbb{k})$ are symmetric monoidal, with the tensor product defined on objects as

$$
\left(X \otimes_{\mathbb{k}} Y\right)^{k}=\bigoplus_{i+j=k} X^{i} \otimes_{\mathbb{k}} Y^{j}, \quad d_{X \otimes_{\mathbb{k}} Y}=d_{X} \otimes \operatorname{Id}_{Y}+\operatorname{Id}_{X} \otimes d_{Y}
$$

and on morphisms $f, g$ by $(f \otimes g)(x \otimes y)=(-1)^{|x||g|} f(x) \otimes g(y)$, with braiding

$$
\tau_{X, Y}: X \otimes_{\mathbb{k}} Y \rightarrow Y \otimes_{\mathbb{k}} X, \quad \tau_{X, Y}(x \otimes y)=(-1)^{|x||y|} y \otimes x .
$$

Finally, for a complex $X$ and $l \in \mathbb{Z}$, we denote by $\Sigma^{l} X$ the complex with

$$
\left(\Sigma^{l} X\right)^{k}=X^{k+l}, \quad d_{\Sigma^{l} X}=(-1)^{l} d_{X} .
$$

In particular, for $l>0$, the translation $\Sigma^{l}$ shifts the complex $X$ to the left by $l$ steps.

2.2. Differential graded categories. A differential $\mathbb{Z}$-graded $\mathbb{k}$-linear category $\mathcal{C}$, or short a $d g$ category is a category enriched in $\mathrm{Ch}(\mathbb{k})$. This means every morphism space $\operatorname{Hom}_{\mathcal{C}}(X, Y)$ is an object in $\mathrm{Ch}(\mathbb{k})$ and composition of morphisms forms chain maps

$$
\operatorname{Hom}_{\mathcal{C}}(Y, Z) \otimes_{\mathbb{k}} \operatorname{Hom}_{\mathcal{C}}(X, Y) \rightarrow \operatorname{Hom}_{\mathcal{C}}(X, Z)
$$

which means that the differentials satisfy the Leibniz rule with respect to composition: $d_{\mathfrak{e}}(f \circ g)=d_{\mathfrak{e}}(f) \circ g+(-1)^{|f|} f \circ d_{\mathfrak{e}}(g)$. Other abelian groups besides $\mathbb{Z}$ can be used for gradings, and later we will consider $\mathbb{Z} \times \mathbb{Z}$-graded complexes with differentials of degree $(0,1)$. In any case, the cohomological degree of a homogenous morphism $f$ will be denoted by $|f|$.

Example 2.1. Any $\mathbb{k}$-linear category can and will be regarded as a dg category with morphism complexes concentrated in cohomological degree zero, thus necessarily with zero differential.

Example 2.2. For any $\mathbb{k}$-linear category $\mathcal{A}$, the category $\operatorname{Ch}(\mathcal{A})\left(\operatorname{resp} \cdot \operatorname{Ch}^{b}(\mathcal{A})\right)$ of (bounded) complexes in $\mathcal{A}$ (whose definition mimics the one of $\mathrm{Ch}(\mathbb{k})\left(\right.$ resp. $\left.\mathrm{Ch}^{b}(\mathbb{k})\right)$ ) is a dg category.

Example 2.3. Any $(\mathrm{dg}) \mathbb{k}$-algebra $A$ can and will be regarded as a dg category with one object $*$ and $\operatorname{End}_{A}(*)=A$.

A morphism $f \in \operatorname{Hom}_{\mathfrak{e}}(X, Y)$ is said to be closed if $d_{\mathfrak{e}}(f)=0$ and exact or nullhomotopic if $f=d_{\mathfrak{e}}(h)$ for some $h \in \operatorname{Hom}_{\mathcal{C}}(X, Y)$, which in this case is called a null-homotopy for $f$. For $f, g \in \operatorname{Hom}_{\mathfrak{e}}^{k}(X, Y)$, we write $f \simeq g$ and say $f$ and $g$ are homotopic if $f-g$ is null-homotopic. 
The cohomology category of $\mathcal{C}$, denoted $H^{0}(\mathcal{C})$ is defined to be the additive category with the same objects as $\mathcal{C}$, and with

$$
\operatorname{Hom}_{H^{0}(\mathcal{C})}(X, Y):=\frac{\left\{f \in \operatorname{Hom}_{\mathfrak{C}}^{0}(X, Y) \mid d_{\mathfrak{C}}(f)=0\right\}}{d_{\mathfrak{C}}\left(\operatorname{Hom}_{\mathcal{C}}^{-1}(X, Y)\right)}
$$

By isomorphism in $\mathcal{C}$ we mean degree zero closed invertible morphisms. If there exists an isomorphism in $\operatorname{Hom}_{\mathcal{C}}(X, Y)$, we write $X \cong Y$. A degree zero closed morphism $f \in \operatorname{Hom}_{\mathcal{C}}(X, Y)$ is said to be a homotopy equivalence if it induces an isomorphism in $H^{0}(\mathrm{C})$, and in this case we write $X \simeq Y$ and say $X$ and $Y$ are homotopy equivalent. If $X \simeq 0$, then we say $X$ is contractible.

For a $\mathrm{dg}$ category $\mathcal{C}$, we denote by $\mathcal{C}^{\text {op }}$ the $\mathrm{dg}$ category with the same objects as $\mathcal{C}$ and with $\operatorname{Hom}_{\mathcal{C o p}}(X, Y):=\operatorname{Hom}_{\mathcal{C}}(Y, X)$, where the composite of $f \in \operatorname{Hom}_{\mathcal{C o p}}(X, Y)$ with $g \in \operatorname{Hom}_{\mathcal{C o p}}(Y, Z)$, denoted $g \circ \circ_{\text {eop }} f$, is given by $(-1)^{|f||g|} f \circ_{\mathcal{C}} g$.

For two dg categories $\mathcal{C}, \mathcal{D}$ we denote by $\mathcal{C} \otimes_{\mathbb{k}} \mathcal{D}$ the category with objects given by pairs of objects $(X, Y)$ for $X \in \mathcal{C}$ and $Y \in \mathcal{D}$ and morphisms given by complexes

$$
\operatorname{Hom}_{\bigotimes_{\otimes_{\mathfrak{k}} \mathcal{D}}}\left((X, Y),\left(X^{\prime}, Y^{\prime}\right)\right):=\operatorname{Hom}_{\mathcal{C}}\left(X, X^{\prime}\right) \otimes_{\mathbb{k}} \operatorname{Hom}_{\mathcal{D}}\left(Y, Y^{\prime}\right)
$$

with composition

$$
(f \otimes g) \circ\left(f^{\prime} \otimes g^{\prime}\right):=(-1)^{|g|\left|f^{\prime}\right|}\left(f \circ f^{\prime}\right) \otimes\left(g \circ g^{\prime}\right) .
$$

Let $\mathcal{C}$ be a dg category. A subcategory $\mathcal{J} \subset \mathcal{C}$ is a collection of objects $\operatorname{Obj}(\mathcal{J}) \subset \operatorname{Obj}(\mathcal{C})$ with hom spaces $\operatorname{Hom}_{\mathcal{J}}\left(X, X^{\prime}\right)$ for $X, X^{\prime} \in \operatorname{Obj}(\mathcal{J})$ being subcomplexes $\operatorname{Hom}_{\mathcal{C}}\left(X, X^{\prime}\right)$, which are closed under composition. The subcategory $\mathcal{J} \subset \mathcal{C}$ is full if $\operatorname{Hom}_{\mathcal{J}}\left(X, X^{\prime}\right)=$ $\operatorname{Hom}_{\mathcal{C}}\left(X, X^{\prime}\right)$ for all $X, X^{\prime} \in \operatorname{Obj}(\mathcal{J})$. The subcategory $\mathcal{J} \subset \mathcal{C}$ is unital if $\operatorname{Id}_{X} \in \mathcal{J}$ whenever $X \in \operatorname{Obj}(\mathcal{J})$. Henceforth all subcategories are unital.

Example 2.4. We will denote by $\mathcal{J} \subset \mathcal{C}$ the subcategory spanned by the identity maps in $\mathcal{C}$. More generally, if $\mathcal{B} \subset \mathcal{C}$ is a full subcategory, then we have $\mathfrak{J}_{\mathcal{B}} \subset \mathcal{C}$, the (unital, but not full) subcategory spanned by the identity morphisms in $\mathcal{C}$ which are contained in $\mathcal{B}$.

2.3. DG functors. If $\mathcal{B}, \mathcal{C}$ are $\operatorname{dg}$ categories, a $\operatorname{dg}$ functor $F: \mathcal{B} \rightarrow \mathcal{C}$ is a functor whose action on hom complexes $\operatorname{Hom}_{\mathcal{B}}(X, Y) \rightarrow \operatorname{Hom}_{\mathcal{C}}(F(X), F(Y))$ is a degree zero chain map. The collection of $\mathrm{dg}$ functors $\mathcal{B} \rightarrow \mathcal{C}$ itself forms a dg category. Objects of this functor category are functors, and morphisms are natural transformations, as defined next. If $F, G$ are functors $\mathcal{B} \rightarrow \mathcal{C}$, a natural transformation $\alpha: F \rightarrow G$ of degree $k$ is an assignment $X \mapsto \alpha_{X} \in \operatorname{Hom}_{\mathfrak{e}}^{k}(F(X), G(X))$ such that

$$
G(f) \circ \alpha_{X}=(-1)^{k|f|} \alpha_{Y} \circ F(f)
$$

for all morphisms $f \in \operatorname{Hom}_{\mathcal{B}}(X, Y)$. The differential of $\alpha$ by definition sends $X \mapsto$ $d_{\mathfrak{e}}\left(\alpha_{X}\right)$ (the naturality of $d\left(\alpha_{X}\right)$ so defined follows from the Leibniz rule).

By an isomorphism of $d g$ functors we mean a degree zero closed invertible natural transformation of $\mathrm{dg}$ functors. If $F, G: \mathcal{B} \rightarrow \mathcal{C}$ are isomorphic, we write $F \cong G$. 
Any dg functor $F: \mathcal{B} \rightarrow \mathcal{C}$ naturally induces a functor between the corresponding homotopy categories $H^{0}(F): H^{0}(\mathcal{B}) \rightarrow H^{0}(\mathcal{C})$.

A dg functor $F: \mathcal{C} \rightarrow \mathcal{D}$ is an equivalence of dg categories if there is a dg functor $G: \mathcal{D} \rightarrow \mathcal{C}$ such that $F \circ G \cong \operatorname{Id}_{\mathcal{D}}$ and $G \circ F \cong \operatorname{Id}_{\mathcal{C}}$.

A dg functor $F: \mathcal{C} \rightarrow \mathcal{D}$ is quasi-fully faithful, if restricts to quasi-isomorphisms on hom complexes, i.e. for every pair of objects $X, Y$ in $\mathcal{E}$, the induced map $H^{\bullet}(F): H^{\bullet}\left(\operatorname{Hom}_{\mathcal{C}}(X, Y)\right) \rightarrow H^{*}\left(\operatorname{Hom}_{\mathcal{D}}(F(X), F(Y))\right)$ is an isomorphism; it is quasiessentially surjective if the functor $H^{0}(F)$ between the respective homotopy categories is essentially surjective. If $F$ is quasi-fully faithful and quasi-essentially surjective, then it is a called a quasi-equivalence.

For many applications in this paper we will need a weaker notion of a functor between $\mathrm{dg}$ categories. If dgcat is the category where the objects are all (small) dg categories and the morphisms are all dg functors, one can define [Tab05, Toë07] the category Hqe as a localization of dgcat with respect to quasi-equivalences. More abstractly, dgcat has a model category structure whose weak equivalences are the quasi-equivalences, and Hqe is the corresponding localization.

Two dg categories $\mathcal{C}$ and $\mathcal{D}$ are called quasi-equivalent if there exist $\mathrm{dg}$ categories $\mathcal{B}_{1}, \ldots, \mathcal{B}_{n}$ and a chain of quasi-equivalences

$$
\mathcal{C} \leftarrow \mathcal{B}_{1} \rightarrow \ldots \leftarrow \mathcal{B}_{n} \rightarrow \mathcal{D} \text {. }
$$

Then $\mathcal{C}$ and $\mathcal{D}$ are quasi-equivalent if and only if they are isomorphic in Hqe.

A quasi-functor between two dg categories is a morphism in Hqe. For example, if we have a dg functor $F: \mathcal{C} \rightarrow \mathcal{B}$ and a quasi-equivalence $G: \mathcal{D} \rightarrow \mathcal{B}$ then $F$ induces a quasi-functor from $\mathcal{C}$ to $\mathcal{D}$. Since quasi-equivalences induces equivalences of homotopy categories, a quasi-functor between $\mathrm{dg}$ categories $\mathcal{C}$ and $\mathcal{D}$ induces an honest functor between the homotopy categories $H^{0}(\mathcal{C}) \rightarrow H^{0}(\mathcal{D})$.

Remark 2.5. Instead of working with Hqe and quasi-functors, one could choose to work with $A_{\infty}$-functors between dg categories. Over a field $\mathbb{k}$, this is essentially an equivalent viewpoint since every quasi-equivalence admits an inverse, which is in general not a dg functor but an $A_{\infty}$-functor [Sei08]. However, we decided to stay away from $A_{\infty}$-functors in this paper.

2.4. Bimodules. If $\mathcal{C}$ and $\mathcal{D}$ are dg categories, a $\mathcal{D}, \mathcal{C}$-bimodule $\mathcal{M}$ is the data of

- for each pair of objects $Y \in \mathcal{D}, X \in \mathcal{C}$, a dg $\mathbb{k}$-module $Y \mathcal{N} X$.

- for each quadruple of objects $Y, Y^{\prime} \in \mathcal{D}$ and $X, X^{\prime} \in \mathcal{C}$, action maps

$$
\operatorname{Hom}_{\mathcal{D}}\left(Y, Y^{\prime}\right) \otimes_{\mathbb{k}} Y \mathcal{M} X \otimes_{\mathbb{k}} \operatorname{Hom}_{\mathcal{C}}\left(X^{\prime}, X\right) \rightarrow Y^{\prime} \mathcal{M} X^{\prime}
$$

satisfying the usual associativity constraints.

The action maps are required to be chain maps of degree zero. This is equivalent to $|f \cdot m \cdot g|=|f|+|m|+|g|$ and the Leibniz rule

$$
d_{\mathfrak{N}}(f \cdot m \cdot g)=d_{\mathcal{D}}(f) \cdot m \cdot g+(-1)^{|f|} f \cdot d_{\mathcal{M}}(m) \cdot g+(-1)^{|f|+|m|} f \cdot m \cdot d_{\mathfrak{C}}(g)
$$

for all $f \in \operatorname{Hom}_{\mathcal{D}}\left(Y, Y^{\prime}\right), m \in Y \mathcal{N} X, g \in \operatorname{Hom}_{\mathcal{C}}\left(X^{\prime}, X\right)$. 
The notation ${ }_{\mathcal{D}} \mathcal{M}_{\mathcal{C}}$ will be used to indicate that $\mathcal{M}$ is a $\mathcal{D}, \mathcal{C}$-bimodule.

A left $\mathcal{C}$-module is the same as a $\mathrm{C}, \mathbb{k}$-bimodule, and a right $\mathrm{C}$-module is the same as a $\mathbb{k}$, $\mathrm{C}$-bimodule, by definition.

Example 2.6. If $\mathcal{C}$ is a $\mathrm{dg}$ category and $X, Y \in \mathcal{C}$ are objects, then we denote

$$
\begin{aligned}
& Y \mathcal{C} X:=\operatorname{Hom}_{\mathcal{C}}(X, Y) \text {, }
\end{aligned}
$$

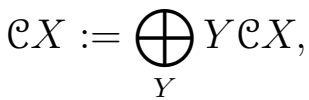

$$
\begin{aligned}
& Y \mathcal{C}:=\bigoplus_{X} Y \circlearrowright X .
\end{aligned}
$$

The composition of morphisms in $\mathcal{C}$ equips $\mathcal{C} X$ with the structure of a left $\mathcal{C}$-module, $Y \mathcal{C}$ with the structure of a right $\mathcal{C}$-module (called the Yoneda modules), and $\mathrm{C}=$ $\bigoplus_{X, Y} Y \mathcal{C} X$ with the structure of a $\mathrm{C}$, $\mathrm{C}$-bimodule (the regular bimodule).

We will use the Yoneda modules and the regular bimodule to streamline notation in certain places.

Remark 2.7. For $\mathrm{dg}$ categories $\mathcal{C}, \mathcal{D}$, the following notions are equivalent:

(1) $\mathcal{D}$, C-bimodules,

(2) left $\mathcal{D} \otimes_{\mathbb{k}} \mathrm{C}^{\mathrm{op}}$-modules,

(3) right $\mathcal{C} \otimes_{\mathbb{k}} \mathcal{D}^{\mathrm{op}}$-modules,

(4) functors $\mathcal{D} \otimes_{\mathbb{k}}$ eop $\rightarrow \mathbb{k}$-dgmod.

However, such identifications necessarily involve choices and hidden signs; for this reason, we will typically not use them.

Given dg categories $\mathcal{B}, \mathcal{C}, \mathcal{D}$ and bimodules ${ }_{\mathcal{D}} \mathcal{M}_{\mathcal{C}}$, ${ }_{e} \mathcal{N}_{\mathcal{B}}$, their tensor product ${ }_{\mathcal{D}}\left(\mathcal{M} \otimes_{\mathcal{e}}\right.$ $\mathcal{N})_{\mathcal{B}}$ is the bimodule with

$$
Z\left(\mathcal{M} \otimes_{\mathfrak{e}} \mathcal{N}\right) X:=\bigoplus_{Y \in \mathcal{C}}(Z \mathcal{M} Y \otimes Y \mathcal{N} X) / \sim
$$

where $\sim$ is the equivalence relation $(m \cdot f) \otimes n \sim m \otimes(f \cdot n)$ for all $m \in Z \mathcal{M} Y$, $f \in Y \mathcal{e} Y^{\prime}, n \in Y^{\prime} \mathcal{N} X$.

Remark 2.8. The category of $\mathcal{C}, \mathcal{C}$-bimodules is monoidal with tensor product as defined above, and monoidal identity given by the regular bimodule $\mathrm{C}$.

Remark 2.9. We often regard $\mathcal{C}$ and $\mathcal{D}$ as the (very big) non-unital algebras

$$
\mathcal{C}=\bigoplus_{X^{\prime}, X \in \mathcal{C}} X^{\prime} \mathcal{C} X, \quad \mathcal{D}=\bigoplus_{Y^{\prime}, Y \in \mathcal{D}} Y^{\prime} \mathcal{D} Y
$$

and a $\mathcal{D}$, $\mathrm{C}$-bimodule $\mathcal{M}$ as the (very big) dg bimodule

$$
\mathcal{M}=\bigoplus_{Y \in \mathcal{D}, X \in \mathcal{C}} Y \mathcal{M} X
$$

In this language an object $X \in \mathcal{C}$ corresponds to the distinguished idempotent $\operatorname{Id}_{X}$ in the big algebra $\bigoplus_{X^{\prime}, X} X^{\prime} \mathcal{C} X$. In this way, essentially all of one's intuition from the usual world of algebras and bimodules carries over into the world of $\mathrm{dg}$ categories and their bimodules. 
2.5. $A_{\infty}$ algebras and deformation retracts. Recall, an $A_{\infty}$-algebra is a graded $\mathbb{k}^{-}$ module $A$ equipped with maps $\mu_{n}: A^{\otimes n} \rightarrow A$ degree $2-n, n \geq 1$, satisfying the following family of identities for $M \geq 1$

$$
\sum_{M=r+s+t}(-1)^{r+s t} \mu_{r+1+t}\left(\operatorname{Id}^{\otimes r} \otimes \mu_{s} \otimes \operatorname{Id}^{\otimes t}\right)=0 .
$$

In particular a dg algebra is an $A_{\infty}$-algebra in which $\mu_{n}$ vanish for $n \neq 1,2$. In this case $\mu_{1}: A \rightarrow A$ is the differential and $\mu_{2}: A \otimes A \rightarrow A$ is honestly associative and satisfies the Leibniz rule

$$
\mu_{1} \circ \mu_{2}-\mu_{2} \circ\left(\mu_{1} \otimes \operatorname{Id}_{A}\right)-\mu_{2} \circ\left(\operatorname{Id}_{A} \otimes \mu_{1}\right)=0
$$

Suppose $X, Y \in \mathcal{C}$ are objects in a dg category. A deformation retract $X \rightarrow Y$ is the data of closed degree zero morphisms $\pi: X \rightarrow Y, \sigma: Y \rightarrow X$ and a degree -1 homotopy $h \in \operatorname{End}^{-1}(X)$ such that

$$
\pi \circ \sigma=\operatorname{Id}_{Y}, \quad d(h)=\operatorname{Id}_{X}-\sigma \circ \pi, \quad h \circ \sigma=0=\pi \circ h .
$$

The following is well known [Kel01, Kad80, Mer99].

Theorem 2.10. If $A$ is a dg algebra and $V$ is a $d g \mathbb{k}$-module, then any deformation retract $A \rightarrow V$ gives $V$ the structure of an $A_{\infty}$-algebra quasi-isomorphic to $A$.

Proof. The construction follows [Mer99]. Let $m: A \otimes A \rightarrow A$ be the multiplication in $A$. We define recursively a sequence of maps $\lambda_{k}: V^{\otimes k} \rightarrow A$ by $\lambda_{2}=m(\sigma \otimes \sigma)$ and

$$
\lambda_{n}=-m\left(\sigma \otimes h \lambda_{n-1}\right)+\sum_{s=2}^{n-2}(-1)^{s+1} m\left(h \lambda_{s} \otimes h \lambda_{n-s}\right)+(-1)^{n+1} m\left(h \lambda_{n-1} \otimes \sigma\right), n \geq 3
$$

Then $\mu_{n}=\pi \circ \lambda_{n}$ defines the structure of a strictly unital $A_{\infty}$-algebra on $V$.

Remark 2.11. Suppose that $A$ and $V$ have an additional grading which is preserved by the differential and the maps $\sigma, \pi$ and $h$. Then the $A_{\infty}$-structure maps on $V$ can be chosen to preserve this grading as well.

Remark 2.12. If $R \subset A$ is a commutative dg subalgebra then the multiplication on $A$ is $R$-bilinear, that is, descends to the map of $R$-bimodules

$$
m:{ }_{R} A \otimes_{R} A_{R} \rightarrow{ }_{R} A_{R} .
$$

Similarly, if $V$ admits the structure of a $\mathrm{dg} R$-bimodule, and the data of the deformation retract (that is $\sigma, \pi$ and $h$ ) can be chosen to be $R$-bilinear, then the $A_{\infty}$-structure maps on $V$ can be chosen to be $R$-linear, in the sense that they descend to the quotient

$$
\mu_{n}:{ }_{R} V \otimes_{R} V \otimes_{R} \cdots \otimes_{R} V_{R} \rightarrow{ }_{R} V_{R} .
$$

This elementary fact is often very useful.

If $\mathbb{k}$ is a field, then $A$ deformation retracts onto its homology $H(A)$ (regarded as a $\mathrm{dg}$ $\mathbb{k}$-module with zero differential), and so $H(A)$ inherits the structure of an $A_{\infty}$-algebra with $\mu_{1}=0$. A differential graded algebra $A$ is called formal if it is quasi-isomorphic 
to its homology $H(A)$. The above discussion shows that a dg algebra $A$ over a field $\mathbb{k}$ is formal if and only if the $A_{\infty}$ structure on $H(A)$ is trivial, that is, $\mu_{k}=0$ for $k>2$.

\section{STANDARD DG CATEGORICAL CONSTRUCTIONS}

In this and the following section we describe the processes of adjoining finite direct sums, suspension, and twists to a dg category.

A functor $F: \mathcal{C} \rightarrow \mathbb{k}$-dgmod is said to be representable if there is an object $X \in \mathcal{C}$ such that $F$ is isomorphic to $\operatorname{Hom}_{\mathcal{C}}(X,-) \operatorname{(or}_{\mathcal{C}}(-, X)$ if $F$ is contravariant).

The $\mathrm{dg}$ category $\mathcal{C}$ is additive if for each finite collection of objects $X_{i} \in \mathcal{C}$ the functor $Y \mapsto \bigoplus_{i} Y \mathcal{C} X_{i}$ is representable. This means $\mathcal{C}$ has finite coproducts, for which we use the symbol $\oplus$.

The dg category $\mathcal{C}$ is suspended (or has suspension) if for each $X \in \mathcal{C}$ and $l \in \mathbb{Z}$ the functor $Y \mapsto \Sigma^{l}(Y \mathcal{C} X)$ is representable. This is equivalent to the existence of an object $\Sigma^{l} X$, an $l$-translate, for every object $X$ of $\mathcal{C}$, together with a given closed degree $l$ invertible morphism $\Sigma^{l} X \rightarrow X$.

Let $\alpha \in \operatorname{End}_{\mathcal{C}}^{1}(X)$ be an endomorphism in $\mathcal{C}$ satisfying the Maurer-Cartan equation $d_{\mathfrak{C}}(\alpha)+\alpha \circ \alpha=0$. Then we have a functor $\phi_{\alpha}: \mathcal{C} \rightarrow \mathbb{k}$-dgmod sending an object $Y$ to the complex $\left(Y \mathcal{C} X, d_{\alpha}\right)$ with twisted differential $d_{\alpha}(f):=d(f)-(-1)^{|f|} f \circ \alpha$.

The category $\mathrm{dg} \mathcal{C}$ is said to have twists if for each Maurer-Cartan element $\alpha$ in $\mathcal{C}$ the functors $\phi_{\alpha}$ is representable.

3.1. Additive suspended envelope. If $\mathcal{C}$ is a $\mathrm{dg}$ category, the additive suspended envelope $\Sigma \mathcal{C}$ of $\mathcal{C}$ is the dg category whose objects are collections $\left\{\Sigma^{a_{i}} X^{i}\right\}_{i \in I}$ where $I \subset \mathbb{Z}$ is a finite set, $X^{i} \in \mathcal{C}$ and $a_{i} \in \mathbb{Z}$. Morphism complexes in $\Sigma \mathcal{C}$ are by definition

$$
\operatorname{Hom}_{\Sigma \mathcal{C}}^{l}\left(\left\{\Sigma^{a_{i}} X^{i}\right\}_{i \in I},\left\{\Sigma^{b_{j}} Y^{j}\right\}_{j \in J}\right)=\prod_{i \in I} \bigoplus_{j \in J} \operatorname{Hom}_{\mathcal{C}}^{l+b_{j}-a_{i}}\left(X^{i}, Y^{j}\right)
$$

with differential

$$
d_{\Sigma \mathcal{e}}\left(\left(f_{j i}\right)_{(j, i) \in J \times I}\right)=\left((-1)^{b_{j}} d_{\mathfrak{C}}\left(f_{j i}\right)\right)_{(j, i) \in J \times I} .
$$

An element of this hom space can be thought of as a $J \times I$ matrix $\left(f_{j i}\right)$ of morphisms $f_{j i} \in \operatorname{Hom}_{\mathcal{C}}\left(X^{i}, Y^{J}\right)$. Composition of morphisms is given by usual matrix multiplication and composition in $\mathcal{C}$.

There is a canonical fully faithful $\mathrm{dg}$ functor $\mathcal{C} \rightarrow \Sigma \mathcal{C}$ defined object-wise by $X \mapsto\{X\}$ (with indexing set $I$ a singleton), and we may identify $\mathcal{C}$ with its image in $\Sigma \mathcal{C}$. It is straightforward to verify that $\Sigma \mathcal{C}$ is additive and suspended (with $\Sigma^{l}$ indicating $l$-translates), and we will henceforth abuse notation by writing

$$
\bigoplus_{i \in I} \Sigma^{a_{i}} X^{i}:=\left\{\Sigma^{a_{i}} X^{i}\right\}_{i \in I} \in \Sigma \mathcal{C} .
$$

We also write 0 for the empty direct sum, corresponding to the case $I=\emptyset$.

It is not hard to check that $\Sigma \Sigma \mathcal{C} \cong \Sigma \mathcal{C}$ and this idempotent property of the assignment $\mathcal{C} \mapsto \Sigma \mathcal{C}$ justifies the name additive suspended envelope. 
Remark 3.1. We also define an additive suspended envelope $\Sigma^{\Pi} \mathcal{C}$ with countable direct products, where the finiteness assumption on the indexing sets $I \subset \mathbb{Z}$ is removed. Homogeneous morphisms in $\Sigma^{\Pi} \mathcal{C}$ are by definition matrices of morphisms in $\mathcal{C}$, each row of which has only finitely many nonzero entries.

3.2. Twisted envelope and pretriangulated hull. The twisted envelope of $\mathcal{C}$ can be constructed explicitly as follows. Let $\mathrm{Tw}(\mathcal{C})$ be the category with objects $\operatorname{tw}_{\alpha}(X)$ where $X \in \mathcal{C}$ and $\alpha \in \operatorname{End}_{\mathfrak{e}}^{1}(X)$ satisfying $d_{\mathfrak{C}}(\alpha)+\alpha^{2}=0$ as above. The morphism complexes in $\mathrm{Tw}(\mathcal{C})$ are by definition

$$
\operatorname{Hom}_{\mathrm{Tw}(\mathcal{C})}\left(\operatorname{tw}_{\alpha}(X), \operatorname{tw}_{\beta}(Y)\right):=\operatorname{Hom}_{\mathcal{C}}(X, Y)
$$

with differential

$$
d_{\mathrm{Tw}(\mathcal{C})}(f)=d_{\mathfrak{e}}(f)+\beta \circ f-(-1)^{|f|} f \circ \alpha .
$$

We say that $\mathcal{C}$ has twists if the obvious fully faithful $\mathrm{dg}$ functor $\mathcal{C} \rightarrow \operatorname{Tw}(\mathcal{C})$ sending $X \mapsto \operatorname{tw}_{0}(X)$ is an equivalence. The natural inclusion $\operatorname{Tw}(\mathcal{C}) \rightarrow \operatorname{Tw}(\mathrm{Tw}(\mathcal{C}))$ sending $\operatorname{tw}_{\alpha}(X) \mapsto \operatorname{tw}_{0}\left(\operatorname{tw}_{\alpha}(X)\right)$ is an equivalence, with an inverse equivalence $\operatorname{Tw}(\operatorname{Tw}(\mathcal{C})) \rightarrow$ $\mathrm{Tw}(\mathcal{C})$ defined by $\operatorname{tw}_{\beta}\left(\operatorname{tw}_{\alpha}(X)\right) \mapsto \operatorname{tw}_{\alpha+\beta}(X)$ (compare with the procedure of taking the total complex of a bicomplex). Thus, $\mathrm{Tw}(\mathcal{C})$ has twists. This idempotent property of the assignment $\mathcal{C} \mapsto \operatorname{Tw}(\mathcal{C})$ justifies our referring to $\operatorname{Tw}(\mathcal{C})$ as the twisted envelope.

Example 3.2. Any additive category $\mathcal{A}$ can be thought of as a dg category with zero differential and trivial grading (all morphisms are placed in degree zero). Then $\operatorname{Tw}(\Sigma \mathcal{A})$ is equivalent to the usual category of bounded complexes $\mathrm{Ch}^{b}(\mathcal{A})$.

If $f \in \operatorname{Hom}_{\mathcal{C}}^{1}(X, Y)$ is a degree 1 closed morphism, then the cone of $f$ is the object $\operatorname{tw}_{\alpha}(X \oplus Y)$ with $\alpha=\left[\begin{array}{ll}0 & 0 \\ f & 0\end{array}\right]$ inside $\operatorname{Tw}(\mathcal{C})$. If instead $f: X \rightarrow Y$ is a degree zero closed morphism and $\mathcal{C}$ has suspension, then we first replace $f$ by a degree 1 closed morphism $\Sigma^{1} X \rightarrow Y$ and apply the previous construction.

Definition 3.3. We say a dg category $\mathcal{C}$ is pretriangulated if it is suspended and closed under taking cones.

The pretriangulated hull $\operatorname{Pretr}(\mathcal{C})$ of a dg category $\mathcal{C}$ is the full subcategory of $\operatorname{Tw}(\Sigma \mathcal{C})$ generated by $\Sigma \mathcal{C}$ under taking mapping cones.

It follows from the discussion above that $\operatorname{Pretr}(\mathcal{C})$ is pretriangulated, and $\mathcal{C}$ itself is pretriangulated if and only if the natural embedding $\mathcal{C} \rightarrow \operatorname{Pretr}(\mathcal{C})$ is an equivalence.

Objects in the pretriangulated hull can be expressed as iterated mapping cones of objects in $\Sigma \mathrm{C}$, also known as one-sided twisted complexes.

Example 3.4. For $X=\operatorname{tw}_{\alpha}\left(\bigoplus_{j \in J} \Sigma^{a_{j}} X^{j}\right)$ we can collect terms with equal shifts, i.e. set $Y^{i}:=\bigoplus_{j: a_{j}=-i} X^{j}$ for $i \in \mathbb{Z}$, and write $\alpha_{j, i} \in \operatorname{Hom}^{1+i-j}\left(Y^{i}, Y^{j}\right)$ for the components of 
the twist. Then $X$ can be illustrated as:

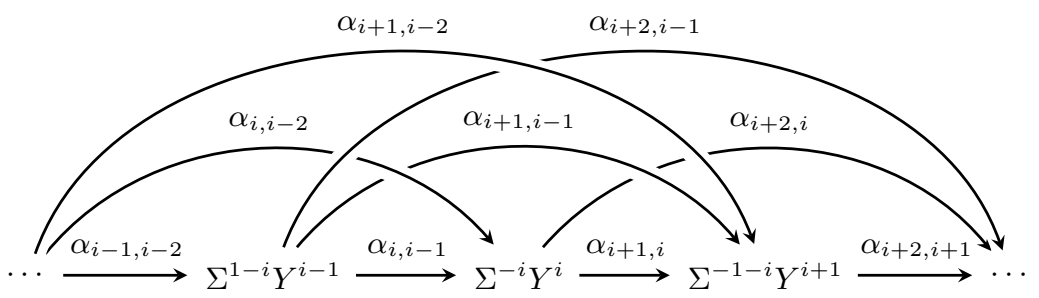

The Maurer-Cartan equation for $\alpha$ now is $(-1)^{j} d_{\mathfrak{C}}\left(\alpha_{j, i}\right)+\sum_{j>k>i} \alpha_{j, k} \circ \alpha_{k, i}=0$.

Remark 3.5. Let $A$ be a dg algebra supported in non-positive degrees, considered as a dg category with one object. Then $\operatorname{Pretr}(A)=\operatorname{Tw}(\Sigma A)$ since every twisted complex is one-sided, i.e. an iterated cone.

3.3. $A_{\infty}$-categories. In this section we briefly discuss $A_{\infty}$-categories and refer the reader to [Kel06, LH03, Sei08] for more complete exposition.

An $A_{\infty}$-category $\mathcal{C}$ consists of a set of objects $\mathrm{Ob}(\mathcal{C})$, a graded vector space $\operatorname{Hom}(X, Y)$ for each pair of objects $X, Y$ and degree $2-d$ composition maps

$$
\mu_{d}: \operatorname{Hom}\left(X_{1}, X_{0}\right) \otimes \cdots \otimes \operatorname{Hom}\left(X_{d}, X_{d-1}\right) \rightarrow \operatorname{Hom}\left(X_{d}, X_{0}\right)
$$

satisfying $A_{\infty}$-equations as in Section 2.5.

An $A_{\infty}$-category $\mathcal{C}$ with one object $X$ is the same data as the $A_{\infty}$-algebra $\operatorname{End}_{\mathfrak{C}}(X)$. If $\mu_{d}=0$ for all $d \geq 3$ in some $A_{\infty}$-category $\mathcal{C}$, then $\mathcal{C}$ is just a dg category with differential $\mu_{1}: \operatorname{Hom}\left(X_{1}, X_{0}\right) \rightarrow \operatorname{Hom}\left(X_{1}, X_{0}\right)$ and composition $\mu_{2}: \operatorname{Hom}\left(X_{1}, X_{0}\right) \otimes$ $\operatorname{Hom}\left(X_{2}, X_{1}\right) \rightarrow \operatorname{Hom}\left(X_{0}, X_{2}\right)$ which in this case is strictly associative.

Given an $A_{\infty}$-category $\mathcal{C}$, we can consider its homotopy category where the objects are the same as in $\mathcal{C}$, and the morphisms are given by the homology with respect to $\mu_{1}$. The composition of morphisms is induced by $\mu_{2}$.

Given objects $X, X^{\prime}$ of an $A_{\infty}$ category $\mathcal{C}$ and $k \in \mathbb{Z}$, we say that $X^{\prime}$ is the $k$-fold suspension of $X$, written $X^{\prime} \cong X[k]$ if there is a morphism $\phi: X \rightarrow X^{\prime}$ of degree $k$ which is closed $\left(\mu_{1}(\phi)=0\right)$ and invertible (there exists $\phi^{\prime}: X^{\prime} \rightarrow X$ of degree $-k$ such that $\mu_{2}\left(\phi, \phi^{\prime}\right)=\operatorname{Id}_{X^{\prime}}$ and $\left.\mu_{2}\left(\phi^{\prime}, \phi\right)=\operatorname{Id}_{X}\right)$.

Given an $A_{\infty}$ category $\mathcal{C}$ we let $\Sigma \mathcal{C}$ denote the closure of $\mathcal{C}$ with respect to finite direct sums and suspensions. Replacing $\mathcal{C}$ by $\Sigma \mathcal{C}$ if necessary, below we will assume that $\mathcal{C}$ is closed under finite direct sum and suspension.

Given an object $X \in \mathcal{C}$, a Maurer-Cartan endomorphism of $X$ is a degree 1 element $\alpha \in \operatorname{End}_{\mathfrak{C}}(X)$ such that

$$
\sum_{d \geq 1} \mu_{d}(\alpha, \ldots, \alpha)=0 .
$$

We say that $\alpha$ is one-sided if there exists a direct sum decomposition $X \cong \bigoplus_{i \in I} X_{i}$ where $I$ is a finite poset, with respect to which $\alpha$ is represented by a strictly lower triangular matrix.

The category $\operatorname{Pretr}(\mathcal{C})$ is the category with 
- objects of $\operatorname{Pretr}(\mathcal{C})$ are formal expressions $\operatorname{tw}_{\alpha}(X)$ in which $\alpha$ is a one-sided twist,

- $\operatorname{Hom}_{\text {Pretr(e) }}\left(\operatorname{tw}_{\alpha}(X), \operatorname{tw}_{\beta}(Y)\right)=\operatorname{Hom}_{\mathcal{C}}(X, Y)$,

- the higher composition $\mu_{d}^{\prime}$ of a sequence of morphisms

$$
\operatorname{tw}_{\alpha_{0}}\left(X_{0}\right) \stackrel{f_{1}}{\longleftarrow} \operatorname{tw}_{\alpha_{1}}\left(X_{1}\right) \stackrel{f_{2}}{\longleftarrow} \ldots \stackrel{f_{d}}{\leftarrow} \operatorname{tw}_{\alpha_{d}}\left(X_{d}\right),
$$

is defined by

$$
\mu_{d}^{\prime}\left(f_{1}, \ldots, f_{d}\right):=\sum_{r_{0}, \ldots, r_{d} \geq 0} \pm \mu_{d+r_{0}+\cdots+r_{d}}(\underbrace{\alpha_{0}, \ldots, \alpha_{0}}_{r_{0}}, f_{1}, \underbrace{\alpha_{1}, \ldots, \alpha_{1}}_{r_{1}}, \ldots, f_{d}, \underbrace{\alpha_{d}, \ldots, \alpha_{d}}_{r_{d}})
$$

(note that the one-sidedness of the Maurer-Cartan elements $\alpha_{i}$ guarantees finiteness of the above sum).

\section{HOMOTOPY IDEMPOTENTS AND THE KAROUBI ENVELOPE}

4.1. Homotopy idempotents. Let $\mathcal{C}$ be a dg category. A homotopy idempotent in $\mathcal{C}$ is a closed endomorphism $e \in \operatorname{End}_{\mathfrak{C}}^{0}(X)$ such that $e^{2} \simeq e$ (i.e. an idempotent in $H^{0}(\mathcal{C})$. We say that $Y$ is an image of $e$ if there exist closed degree zero morphisms $\sigma: Y \rightarrow X$, $\pi: X \rightarrow Y$ such that

$$
\pi \circ \sigma \simeq \operatorname{Id}_{Y}, \quad \sigma \circ \pi \simeq e .
$$

Lemma 4.1. Images of homotopy idempotents satisfy the following basic properties:

(1) Suppose $e_{1}, e_{2} \in \operatorname{End}_{\mathfrak{C}}(X)$ are homotopy idempotents with $e_{1} \simeq e_{2}$. If $Y_{i}$ is an image of $e_{i}(i=1,2)$ then $Y_{1} \simeq Y_{2}$. In particular the image of a homotopy idempotent is unique up to homotopy equivalence.

(2) If $Y$ is an image of a homotopy idempotent $e \in \operatorname{End}_{\mathcal{C}}(X)$ then any homotopy idempotent $e^{\prime} \in \operatorname{End}_{\mathcal{C}}(Y)$ determines a homotopy idempotent $e^{\prime \prime} \in \operatorname{End}_{\mathfrak{C}}(X)$ with the property that $Z$ is an image of $e^{\prime}$ if and only if it is an image of $e^{\prime \prime}$.

(3) If $Y_{0}, Y_{1}$ are the images of homotopy idempotents $e_{i} \in \operatorname{End}_{\mathfrak{e}}\left(X_{i}\right)(i=0,1)$ then

$$
\operatorname{Hom}_{H^{0}(\mathcal{e})}\left(Y_{0}, Y_{1}\right) \cong e_{1} \operatorname{Hom}_{H^{0}(\mathcal{C})}\left(X_{0}, X_{1}\right) e_{0}
$$

Proof. Exercise.

Definition 4.2. We say that $\mathcal{C}$ is homotopy idempotent complete if $H^{0}(\mathcal{C})$ is idempotent complete, i.e. each homotopy idempotent in $\mathcal{C}$ has an image.

Our goal in this section is to construct the homotopy Karoubi envelope $\operatorname{Kar}^{d g}(\mathcal{C})$ and prove the following.

Theorem 4.3. Every $d g$ category $\mathcal{C}$ admits an embedding $\mathcal{C} \hookrightarrow \operatorname{Kar}^{d g}(\mathcal{C})$ into a homotopy idempotent complete dg category characterized up to quasi-equivalence by the following universal property: if $\mathcal{D}$ is a homotopy idempotent complete dg category equipped with a dg 
functor $\mathcal{C} \rightarrow \mathcal{D}$, then there is unique morphism (quasi-functor) $\operatorname{Kar}^{d g}(\mathcal{C}) \rightarrow \mathcal{D}$ in $\mathbf{H q e}$ such that the following diagram commutes:

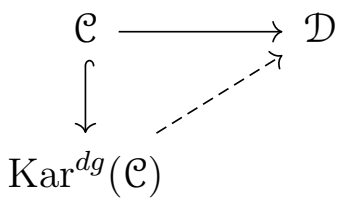

Furthermore, $\mathrm{e}$ is idempotent complete if and only if the canonical functor $\mathrm{e} \rightarrow \operatorname{Kar}^{d g}(\mathrm{C})$ is a quasi-equivalence.

4.2. $A_{\infty}$-idempotents. Note that if $e_{0} \in \operatorname{End}_{\mathcal{C}}^{0}(X)$ is a homotopy idempotent and $h_{0} \in \operatorname{End}_{\mathfrak{e}}^{-1}(X)$ satisfies $d_{\mathfrak{e}}\left(h_{0}\right)=e_{0} \circ\left(\operatorname{Id}_{X}-e_{0}\right)$, then $e_{0} \circ h_{0}-h_{0} \circ e_{0}$ is automatically closed. This morphism obstructs certain constructions, and it is natural to require it to be null-homotopic (as we will see below, one can choose a homotopy $h_{0}$ such that this holds, but not every homotopy satisfies this condition), via an endomorphism we will denote $e_{1}$. There is a higher family of obstructions which is natural to require to be trivial, via homotopies $e_{k}, h_{k}$ for $k \in \mathbb{Z}_{\geq 1}$. This results in the notion of an $A_{\infty}$-idempotent or idempotent up to coherent homotopy, which we describe next.

Definition 4.4. An $A_{\infty}$-idempotent in $\mathcal{C}$ is a triple $(X, \underline{e}, \underline{h})$, consisting of an object $X$, and a collection of endomorphisms $\underline{e}=\left\{e_{k} \in \operatorname{End}_{\mathcal{C}}^{-2 k}(X)\right\}_{k=0}^{\infty}, \underline{h}=\left\{h_{k} \in\right.$ $\left.\operatorname{End}_{\mathfrak{C}}^{-1-2 k}(X)\right\}_{k=0}^{\infty}$ satisfying

$$
\begin{aligned}
d\left(e_{k}\right) & =\sum_{i+j=k-1}\left(e_{i} h_{j}-h_{i} e_{j}\right) \\
d\left(h_{k}\right) & =e_{k}-\sum_{i+j=k} e_{i} e_{j}-\sum_{i+j=k-1} h_{i} h_{j}
\end{aligned}
$$

The complement of the $A_{\infty}$-idempotent $(X, \underline{e}, \underline{h})$ is the $A_{\infty}$ idempotent $(X, \underline{e}, \underline{h})^{\perp}:=$ $\left(X, \underline{e}^{\perp}, \underline{h}^{\perp}\right)$ where

$$
e_{0}^{\perp}=\operatorname{Id}_{X}-e_{0}, \quad e_{k}^{\perp}=-e_{k} \quad(k \geq 1), \quad h_{k}^{\perp}=h_{k} \quad(k \geq 0) .
$$

Verification that this defines an $A_{\infty}$-idempotent is left to the reader.

Definition 4.5. If $(X, \underline{e}, \underline{h})$ is an $A_{\infty}$-idempotent, then let $Z(X, \underline{e}, \underline{h}) \in \operatorname{Tw}\left(\Sigma^{\Pi} \mathcal{C}\right)$ denote the twisted complex of the form $\operatorname{tw}_{\delta}\left(\prod_{k \geq 0} \Sigma^{-k} X\right)$ and differential $\delta$ given in terms of components by

$$
\delta_{j i}= \begin{cases}-e_{k}^{\perp} & \text { for } i \text { even, } j=i+1+2 k \\ h_{k} & \text { for } i \text { even, } j=i+2 k+2 \\ e_{k} & \text { for } i \text { odd, } j=i+1+2 k \\ -h_{k} & \text { for } i \text { odd, } j=i+2 k+2\end{cases}
$$

(recall that $-e_{k}^{\perp}=e_{k}$ for $k \geq 1$ ). 
This twisted complex can be visualized as

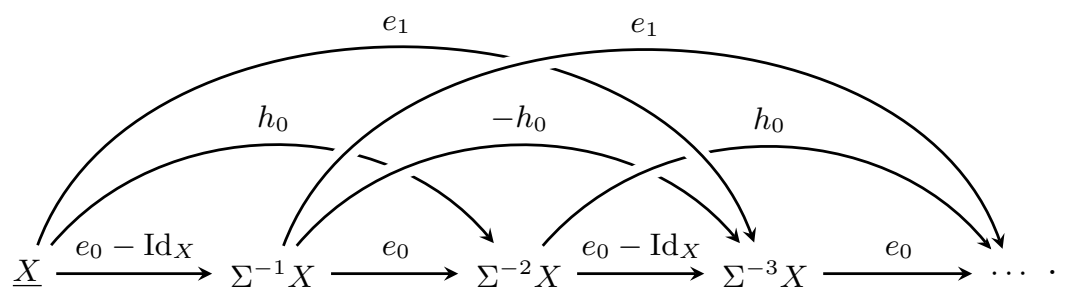

with length $>3$ components of the differential not pictured.

Example 4.6. The identity of $X$ gives an $A_{\infty}$-idempotent with $e_{0}=\operatorname{Id}_{X}$ and $e_{k+1}=$ $0=h_{k}$ for $k \geq 0$. The resulting twisted complex

$$
Z\left(X, \operatorname{Id}_{X}, 0\right)=\underline{X} \stackrel{0}{\longrightarrow} \Sigma^{-1} X \stackrel{\mathrm{Id}_{X}}{\longrightarrow} \Sigma^{-2} X \stackrel{0}{\longrightarrow} \Sigma^{-3} X \stackrel{\mathrm{Id}_{X}}{\longrightarrow} \cdots
$$

is homotopy equivalent to $X$ after the cancellation of contractible summands.

We wish to prove that $Z(X, \underline{e}, \underline{h})$ is a well-defined twisted complex, and is an image of of $e_{0}$. To prove this requires a considerable amount of bookkeeping, for which it is useful to consider the generating functions $e(z)=\sum_{k \geq 0} e_{k} z^{k}$ and $h(z)=\sum_{k \geq 0} h_{k} z^{k}$ where $z$ is a formal indeterminate of degree 2. Below we work an abstract dg category which formalizes the relations satisfied by these generating functions.

\subsection{Abstract $A_{\infty}$-idempotents and their images.}

Definition 4.7. Let $z$ be a formal indeterminate of cohomological degree 2 . Let $\mathcal{R}_{\text {idem }}$ be the $\mathbb{k} \llbracket z \rrbracket$-linear dg category with one object $\mathbf{X}$ whose endomorphism complex is freely generated by endomorphisms $e, h \in \operatorname{End}_{\mathcal{P}_{\text {idem }}}(\mathbf{X})$ satisfying

$$
\operatorname{deg}(e)=0, \quad \operatorname{deg}(h)=-1,
$$

$$
\begin{aligned}
d(e) & =z(e h-h e) \\
d(h) & =e-e^{2}-z h^{2},
\end{aligned}
$$

extended to arbitrary morphisms by the Leibniz rule.

To check that $\mathcal{R}_{\text {idem }}$ is a dg category, one must check that $d^{2}=0$ on all morphisms. It suffices to check on the generating morphisms, which is straightforward. For instance to verify that $d^{2}(e)=0$ it suffices to verify that $d(e h)=d(h e)$, which follows from the computations

$$
d(e h)=d(e) h+e d(h)=z(e h-h e) h+e\left(e-e^{2}-z h^{2}\right)=e^{2}-e^{3}-z h e h
$$

and

$$
d(h e)=d(h) e-h d(e)=\left(e-e^{2}-z h^{2}\right) e-z h(e h-h e)=e^{2}-e^{3}-z h e h .
$$

The proof that $d^{2}(h)=0$ is equally straightforward. 
The notion of an $A_{\infty}$-idempotent in $\mathcal{C}$ can now be described as follows. Consider the $\mathrm{dg}$ category $\mathrm{C} \llbracket z \rrbracket$ with the same objects as $\mathcal{C}$, and morphism complexes

$$
\operatorname{Hom}_{\mathbb{C} \llbracket z \rrbracket}(X, Y):=\operatorname{Hom}_{\mathcal{C}}(X, Y) \otimes_{\mathbb{k}} \mathbb{k} \llbracket z \rrbracket .
$$

Then an $A_{\infty}$-idempotent in $\mathcal{C}$ is equivalent to a dg functor $\mathcal{R}_{\text {idem }} \rightarrow \mathfrak{C} \llbracket z \rrbracket$. The image of $\mathrm{X}$ in $\mathcal{C}$ is an object $X \in \mathcal{C}$, and the images of $e, h$ are formal series of morphisms $h(z)$,

$$
\begin{array}{cl}
e(z)=\sum_{k \geq 0} e_{k} z^{k} & \left(e_{k} \in \operatorname{End}_{\mathcal{C}}^{-2 k}(X)\right), \\
h(z)=\sum_{k \geq 0} h_{k} z^{k} & \left(h_{k} \in \operatorname{End}_{\mathcal{C}}^{-1-2 k}(X)\right),
\end{array}
$$

satisfying the identities (6a), (6b). In terms of components, this yields (5a),(5b).

The following is responsible for the notion of complementary $A_{\infty}$-idempotents.

Lemma 4.8. There is an automorphism of $\mathcal{R}_{\text {idem }}$ which sends $e \mapsto 1-e$ and fixes $h$.

Now we have an analogue of Definition 4.5.

Definition 4.9. Let $Z(e, h)$ denote the twisted complex $\operatorname{tw}_{\alpha}\left(\mathbf{X} \oplus \Sigma^{-1} \mathbf{X}\right)$ where

$$
\alpha=\left[\begin{array}{cc}
z h & z e \\
e-1 & -z h
\end{array}\right] .
$$

We also have an analogue of $X$, viewed through Example 4.6.

Definition 4.10. Let $Z(1,0) \in \operatorname{Tw}\left(\Sigma \mathcal{R}_{\text {idem }}\right)$ denote the twisted complex $\operatorname{tw}_{\beta}\left(\mathbf{X} \oplus \Sigma^{-1} \mathbf{X}\right)$ where

$$
\beta=\left[\begin{array}{ll}
0 & z \\
0 & 0
\end{array}\right] .
$$

Our goal is show that $Z(e, h)$ is the image of a homotopy idempotent acting on $Z(1,0)$.

Lemma 4.11. The object $Z(1,0)=\operatorname{tw}_{\beta}\left(\mathbf{X} \oplus \Sigma^{-1} \mathbf{X}\right)$ is a well-defined twisted complex in $\operatorname{Tw}\left(\Sigma^{\Pi} \mathcal{R}_{\text {idem }}\right)$, and the following defines a homotopy idempotent $E$ acting on $Z(1,0)$ :

$$
E:=\left[\begin{array}{cc}
e & 0 \\
h e-e h & e
\end{array}\right] .
$$

Proof. The first statement is clear since $d(\beta)=0=\beta^{2}$. For the second statement, let

$$
H:=\left[\begin{array}{cc}
h & 0 \\
h^{2} & -h
\end{array}\right],
$$

regarded as an endomorphism of $\mathbf{X} \oplus \Sigma^{-1} \mathbf{X}$. It is straightforward to check that

$$
d(H)+\beta H+\beta H=E-E^{2} .
$$


For this, one must keep in mind that a sign appears in the bottom row of the matrix representing $d(H)$, due to the signs involved in differentiating morphisms in the suspended envelope (4):

$$
d\left(\left[\begin{array}{cc}
h & 0 \\
h^{2} & -h
\end{array}\right]\right)=\left[\begin{array}{cc}
d(h) & 0 \\
-d\left(h^{2}\right) & d(h)
\end{array}\right]
$$

Lemma 4.12. The object $Z(e, h)=\operatorname{tw}_{\alpha}\left(\mathbf{X} \oplus \Sigma^{-1} \mathbf{X}\right)$ from Definition 4.9 is a well-defined twisted complex in $\mathrm{Tw}\left(\Sigma^{\Pi} \mathcal{R}_{\text {idem }}\right)$; moreover $Z(e, h)$ is an image of the homotopy idempotent $E$ acting on $Z(1,0)$ (from Lemma 4.11).

Proof. To show that $Z(e, h)$ is a well-defined twisted complex we must check that $\alpha \in \operatorname{End}_{\Sigma \mathcal{R}_{\text {idem }}}\left(\mathbf{X} \oplus \Sigma^{-1} \mathbf{X}\right)$ satisfies the Maurer-Cartan equation

$$
\left[\begin{array}{ll}
z d(h) & z d(e) \\
-d(e) & z d(h)
\end{array}\right]+\left[\begin{array}{cc}
z h & z e \\
e-1 & -z h
\end{array}\right]\left[\begin{array}{cc}
z h & z e \\
e-1 & -z h
\end{array}\right]=0
$$

This is easily verified.

Now, define maps $\sigma: Z(e, h) \rightarrow Z(1,0)$ and $\pi: Z(1,0) \rightarrow Z(e, h)$ by the matrices

$$
\sigma=\left[\begin{array}{ll}
1 & 0 \\
h & e
\end{array}\right], \quad \pi=\left[\begin{array}{cc}
e & 0 \\
-h & 1
\end{array}\right] .
$$

Observe that $\sigma \circ \pi=E$. To check $\sigma$ is closed is a computation:

$$
\left[\begin{array}{cc}
0 & 0 \\
-d(h) & -d(e)
\end{array}\right]+\left[\begin{array}{ll}
0 & z \\
0 & 0
\end{array}\right]\left[\begin{array}{ll}
1 & 0 \\
h & e
\end{array}\right]-\left[\begin{array}{ll}
1 & 0 \\
h & e
\end{array}\right]\left[\begin{array}{cc}
z h & z e \\
e-1 & -z h
\end{array}\right]=0
$$

and that $\pi$ is closed is the computation

$$
\left[\begin{array}{ll}
d(e) & 0 \\
d(h) & 0
\end{array}\right]+\left[\begin{array}{cc}
z h & z e \\
e-1 & -z h
\end{array}\right]\left[\begin{array}{cc}
e & 0 \\
-h & 1
\end{array}\right]-\left[\begin{array}{cc}
e & 0 \\
-h & 1
\end{array}\right]\left[\begin{array}{ll}
0 & z \\
0 & 0
\end{array}\right]=0,
$$

both of which are straightforward.

It remains to show that $\pi \circ \sigma \simeq \operatorname{Id}_{Z(e, h)}$. Observe that

$$
\pi \circ \sigma=\left[\begin{array}{cc}
e & 0 \\
-h & 1
\end{array}\right]\left[\begin{array}{ll}
1 & 0 \\
h & e
\end{array}\right]=\left[\begin{array}{ll}
e & 0 \\
0 & e
\end{array}\right]
$$

Now, let $K=\left[\begin{array}{ll}0 & 1 \\ 0 & 0\end{array}\right] \in \operatorname{End}_{\Sigma \mathcal{R}_{\text {idem }}}\left(\mathbf{X} \oplus \Sigma^{-1} \mathbf{X}\right)$. The following computes $d(K)+\alpha K+K \alpha$ :

$$
d\left(\left[\begin{array}{ll}
0 & 1 \\
0 & 0
\end{array}\right]\right)+\left[\begin{array}{cc}
z h & z e \\
e-1 & -z h
\end{array}\right]\left[\begin{array}{ll}
0 & 1 \\
0 & 0
\end{array}\right]+\left[\begin{array}{ll}
0 & 1 \\
0 & 0
\end{array}\right]\left[\begin{array}{cc}
z h & z e \\
e-1 & -z h
\end{array}\right]=\left[\begin{array}{cc}
e-1 & z h-z h \\
0 & e-1
\end{array}\right],
$$

which shows that $\pi \circ \sigma \simeq \operatorname{Id}_{Z(e, h)}$. This completes the proof that $Z(e, h)$ is an image of the idempotent $E$ acting on $Z(1,0)$. 
The twisted complexes $Z(1,0)$ and $Z(e, h)$ and the maps relating them can be pictured diagrammatically as

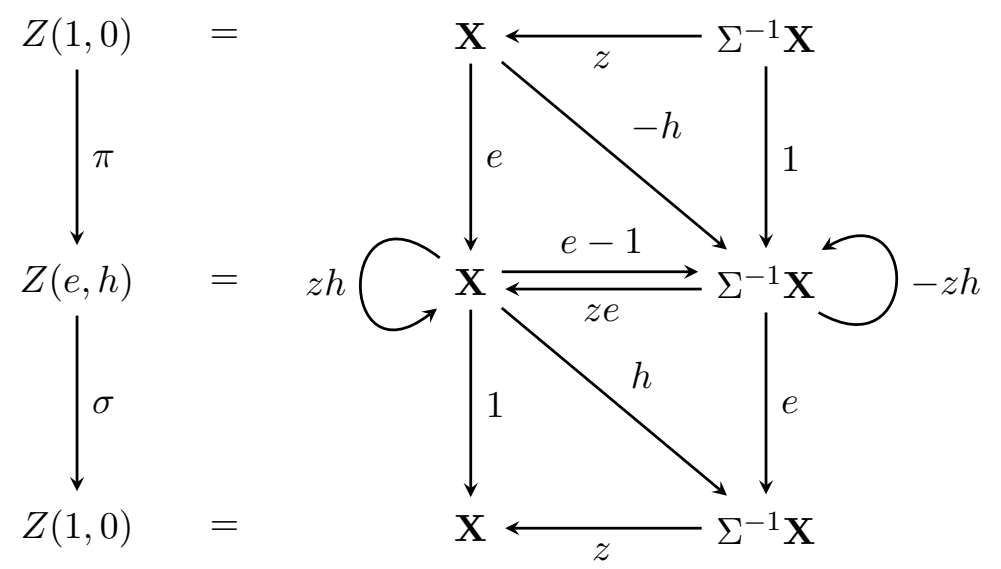

Suppose $(X, \underline{e}, \underline{h})$ is an $A_{\infty}$-idempotent and $\Phi: \mathcal{R}_{\text {idem }} \rightarrow \mathcal{C} \llbracket z \rrbracket$ the corresponding dg functor. We may regard $\mathfrak{C} \llbracket z \rrbracket$ as a (non-full) subcategory of $\Sigma^{\Pi} \mathcal{C}$ via the functor sending $X \mapsto X \llbracket z \rrbracket:=\prod_{k \geq 0} \Sigma^{-2 k} X$ with the formal endomorphism $z$ given by the rightward shift on $X \llbracket z \rrbracket=X \times \Sigma^{-2} X \times \Sigma^{-4} X \times \cdots$.

Thus the images of the abstract twisted complexes $Z(1,0)$ and $Z(e, h)$ under $\Phi$ can be viewed as twisted complexes in $\operatorname{Tw}\left(\Sigma^{\Pi} \mathcal{C}\right)$. A moment's thought confirms that these twisted complexes are precisely $Z\left(X, \operatorname{Id}_{X}, 0\right)$ from Example 4.6 and $Z(X, \underline{e}, \underline{h})$ from Definition 4.5. To see this, note that half terms in $Z(X, \underline{e}, \underline{h})$ yield a copy of $X \llbracket z \rrbracket=\prod_{k \geq 0} \Sigma^{-2 k} X$, while the other half yield a copy of $\Sigma^{-1} X \llbracket z \rrbracket=\prod_{k \geq 0} \Sigma^{-2 k-1} X$.

Since $Z\left(\bar{X}, \operatorname{Id}_{X}, 0\right) \simeq X$, Lemma 4.12 shows that $Z(X, \underline{e}, \underline{h})$ is the image of some homotopy idempotent acting on $X$. It is not hard to see that this homotopy idempotent is $e_{0}$, thereby proving the following.

Proposition 4.13. Let $(X, \underline{e}, \underline{h})$ be an $A_{\infty}$-idempotent in $\mathrm{C}$. The object $Z(X, \underline{e}, \underline{h})$ is a welldefined twisted complex in $\operatorname{Tw}\left(\Sigma^{\Pi} \mathcal{C}\right)$; moreover this twisted complex is an image of the homotopy idempotent $e_{0}$ acting on $X$.

Remark 4.14. The complex $Z(X, \underline{e}, \underline{h})$ has a natural endomorphism $z$ representing the 2-periodicity in this construction. This endomorphism is null-homotopic (by an explicit homotopy), so $z^{k}$ is null homotopic for all $k \geq 1$ as well.

The cone of $z^{k}$ is homotopy equivalent to a finite twisted complex (a truncated version of $Z(X, \underline{e}, \underline{h}))$, which by the above represents the image of $e_{0}$ acting on $X \oplus$ $\Sigma^{2 k-1} X$.

4.4. From homotopy idempotents to $A_{\infty}$-idempotents. Finally, we show that any homotopy idempotent $e$ in $\mathcal{C}$ can be given the structure of an $A_{\infty}$-idempotent. This is well known to experts, but we will give an explicit construction of the higher homotopies following ideas of Seidel [Sei08, Lemma 4.2]. See [BN93, Propositions 3.2 and 3.4] for an alternative proof. We were not able to find explicit formulas for $\underline{e}$ and $\underline{h}$ in the literature. 
Proposition 4.15. Suppose that $e, h \in \operatorname{End}_{\mathcal{C}}(X)$ are such that e is degree zero and closed, and $h$ is degree -1 and satisfies $d_{\mathfrak{C}}(h)=e^{2}-e$. Then there exist endomorphisms $h^{(k)} \in \operatorname{End}^{-k}(X)$ for $k \geq 1$ such that $h^{(0)}=1-e, h^{(1)}=h$ and

$$
d\left(h^{(k)}\right)=\sum_{i=0}^{k-1}(-1)^{i} h^{(i)} h^{(k-1-i)}+ \begin{cases}-h^{(k-1)} & \text { if } k \text { is odd } \\ 0 & \text { if } k \text { is even } .\end{cases}
$$

Corollary 4.16. Suppose that $e, h \in \operatorname{End}_{\mathcal{C}}(X)$ are such that $e$ is degree zero and closed, and $h$ is degree -1 and satisfies $d_{\mathfrak{C}}(h)=e-e^{2}$. Let $h^{(k)}$ denote the morphisms obtained from Proposition 4.15 starting at $(e,-h)$. We define $e_{0}:=e$ and $e_{k}=(-1)^{k+1} h^{(2 k)}$ for $k \geq 1$, as well as $h_{k}:=(-1)^{k+1} h^{(2 k+1)}$ for $k \geq 0$. Then $(X, \underline{e}, \underline{h})$ is an $A_{\infty}$ idempotent in $\mathrm{C}$.

Proof. It is straightforward that these morphisms satisfy the equations from Definition 4.4.

Proof of Proposition 4.15. We will construct $h^{(n)}$ inductively. Recall that $h^{(0)}=1-e$. Suppose that we found $h^{(1)}, \ldots, h^{(n-1)}, h_{\text {temp }}^{(n)}$ such that

$$
\begin{aligned}
& d\left(h^{(k)}\right)=\sum_{i=0}^{k-1}(-1)^{i} h^{(i)} h^{(k-1-i)}+\left\{\begin{array}{ll}
-h^{(k-1)} & \text { if } k \text { is odd } \\
0 & \text { if } k \text { is even. }
\end{array}, \quad 1 \leq k \leq n-1\right. \\
& d\left(h_{\text {temp }}^{(n)}\right)=\sum_{i=0}^{n-1}(-1)^{i} h^{(i)} h^{(n-1-i)}+ \begin{cases}-h^{(n-1)} & \text { if } n \text { is odd } \\
0 & \text { if } n \text { is even. }\end{cases}
\end{aligned}
$$

For $n=1$, this follows from the assumptions of the proposition if we set $h_{\text {temp }}^{(1)}=h$. Our goal is to find an $h_{\text {temp }}^{(n+1)}$ and a closed $y_{n}$, such that (8) will be satisfied for $n \mapsto n+1$ if we set $h^{(n)}=h_{\text {temp }}^{(n)}+y_{n}$. Moreover, (7) will then hold for $k=n$ since $y_{n}$ is closed. To this end, we define

$$
\begin{aligned}
& x_{n}=h^{(0)} h_{\mathrm{temp}}^{(n)}+\sum_{i=1}^{n-1}(-1)^{i} h^{(i)} h^{(n-i)}+(-1)^{n} h_{\mathrm{temp}}^{(n)} h^{(0)}+ \begin{cases}-h_{\mathrm{temp}}^{(n)} & \text { if } n \text { is even } \\
0 & \text { if } n \text { is odd } .\end{cases} \\
& q_{n}=-h^{(1)} h_{\mathrm{temp}}^{(n)}+\sum_{i=2}^{n-1}(-1)^{i} h^{(i)} h^{(n+1-i)}+(-1)^{n} h_{\mathrm{temp}}^{(n)} h^{(1)}
\end{aligned}
$$

It is not hard to check that $d\left(x_{n}\right)=0$. For $x \in \operatorname{End}_{\mathcal{C}}(X)$ we define

$$
A(x)=e x-x e, B(x)=e x-x(1-e) .
$$

We then set $y_{n}=B\left(x_{n}\right)$ if $n$ is even, and $y_{n}=A\left(x_{n}\right)$ if $n$ is odd. In either case we again have $d\left(y_{n}\right)=0$. Further, one can check that $d\left(q_{n}\right)=A\left(x_{n}\right)$ if $n$ is even, and $d\left(q_{n}\right)=B\left(x_{n}\right)$ if $n$ is odd, which helps to verify:

$$
\begin{aligned}
& B\left(y_{n}\right)=B\left(B\left(x_{n}\right)\right)=d\left(h x_{n}+3 x_{n} h+2 q_{n} e-q_{n}\right)+x_{n} \quad \text { if } n \text { is even } \\
& A\left(y_{n}\right)=A\left(A\left(x_{n}\right)\right)=d\left(h x_{n}-3 x_{n} h-2 q_{n} e+q_{n}\right)+x_{n} \quad \text { if } n \text { is odd. }
\end{aligned}
$$


Now we set $h^{(n)}=h_{\text {temp }}^{(n)}+y_{n}$ and $h_{\text {temp }}^{(n+1)}=-\left(h x_{n}+3 x_{n} h+2 q_{n} e-q_{n}\right)$ if $n$ is even, and $h_{\text {temp }}^{(n+1)}=-\left(h x_{n}-3 x_{n} h-2 q_{n} e+q_{n}\right)$ if $n$ is odd. Let us check that (8) is now satisfied for $n \mapsto n+1$. We only consider the case of odd $n+1$, as the other one is analogous.

$$
\begin{aligned}
& d\left(h_{\mathrm{temp}}^{(n+1)}\right)=x_{n}-B\left(y_{n}\right)=h^{(0)} h_{\mathrm{temp}}^{(n)}+\sum_{i=1}^{n-1}(-1)^{i} h^{(i)} h^{(n-i)} \quad+h_{\mathrm{temp}}^{(n)} h^{(0)} \quad-h_{\mathrm{temp}}^{(n)} \\
& +h^{(0)} y_{n} \quad+y_{n} h^{(0)}-y_{n} \\
& =h^{(0)} h^{(n)} \quad+\sum_{i=1}^{n-1}(-1)^{i} h^{(i)} h^{(n-i)}+h^{(n)} h^{(0)} \quad-h^{(n)}
\end{aligned}
$$

where we have used $-B\left(y_{n}\right)=-e y_{n}-y_{n} e+y_{n}=h^{(0)} y_{n}+y_{n} h^{(0)}-y_{n}$.

\subsection{The Karoubi envelope.}

Definition 4.17. For a dg category $\mathcal{C}$ we define the $\mathrm{dg}$ Karoubi envelope $\operatorname{Kar}^{d g}(\mathcal{C})$ as the full dg subcategory of $\operatorname{Tw}\left(\Sigma^{\Pi} \mathcal{C}\right)$ with objects the twisted complexes homotopy equivalent to $Z(X, \underline{e}, \underline{h})$ for some $A_{\infty}$-idempotent $(X, \underline{e}, \underline{h})$ in $\Sigma \mathcal{C}$.

Note that by Example 4.6, we have $\mathcal{C} \hookrightarrow \operatorname{Kar}^{d g}(\mathcal{C})$.

Lemma 4.18. The category $\operatorname{Kar}^{d g}(\mathcal{C})$ is homotopy idempotent complete.

Proof. If $e_{0} \in \operatorname{End}_{\mathcal{C}}(X)$ is a homotopy idempotent, then $e_{0}$ admits a lift to an $A_{\infty}$ idempotent by Proposition 4.15, and Proposition 4.13 constructs an image of $e_{0}$.

On the other hand, every object of $\operatorname{Kar}^{d g}(\mathcal{C})$ is the image of some idempotent in $H^{0}(\mathcal{C})$ by construction, and conversely every idempotent in $\mathcal{C}$ has an image in $\operatorname{Kar}^{d g}(\mathcal{C})$. So if $Y \in \operatorname{Kar}^{d g}(\mathcal{C})$ is the image of a homotopy idempotent $e_{0} \in \operatorname{End}_{\mathcal{C}}(X)$ then all images of all homotopy idempotents in $\operatorname{End}_{\operatorname{Kar}^{d g}(\mathcal{C})}(Y)$ can be constructed as images of some induced homotopy idempotents $e_{0}^{\prime \prime} \in \operatorname{End}_{\mathcal{C}}(X)$ by part (2) of Lemma 4.1 .

Lemma 4.19. There is an equivalence of additive categories $H^{0}\left(\operatorname{Kar}^{d g}(\mathcal{C})\right) \simeq \operatorname{Kar} H^{0}(\mathcal{C})$, where the latter denotes the usual idempotent completion of the additive category $H^{0}(\mathrm{C})$.

Proof. Lemma 4.18 implies that $H^{0}\left(\operatorname{Kar}^{d g}(\mathcal{C})\right)$ is idempotent complete in the usual sense, for additive categories. Now, the canonical functor $H^{0}(\mathcal{C}) \rightarrow H^{0}\left(\operatorname{Kar}^{d g}(\mathcal{C})\right)$ induces a functor on the Karoubi envelope $\operatorname{Kar}\left(H^{0}(\mathcal{C})\right) \rightarrow H^{0}\left(\operatorname{Kar}^{d g}(\mathcal{C})\right)$ because the latter is Karoubian. This functor is essentially surjective because every object in $\operatorname{Kar}^{d g}(\mathcal{C})$ is the image of some homotopy idempotent acting on some object of $\mathcal{C}$, and fully faithful by part (3) of Lemma 4.1.

Lemma 4.20. Adg category $\mathcal{D}$ is homotopy idempotent complete if and only if the natural functor $\mathcal{D} \rightarrow \operatorname{Kar}^{d g}(\mathcal{D})$ is a quasi-equivalence.

Proof. Assume that $H^{0}(\mathcal{D})$ is idempotent complete. Then $H^{0}(\Sigma \mathcal{D})$ is also idempotent complete. By Lemma 4.19 the natural functor $\Sigma \mathcal{D} \rightarrow \operatorname{Kar}^{d g}(\Sigma \mathcal{D})$ induces an 
equivalence $H^{0}(\Sigma \mathcal{D}) \rightarrow H^{0}\left(\operatorname{Kar}^{d g}(\Sigma \mathcal{D})\right) \simeq \operatorname{Kar}\left(H^{0}\left(\Sigma^{\mathcal{D}}\right)\right)$, hence $\mathcal{D} \rightarrow \operatorname{Kar}^{d g}(\mathcal{D})$ is a quasi-equivalence.

For the converse, suppose that the canonical functor $\mathcal{D} \rightarrow \operatorname{Kar}^{d g}(\mathcal{D})$ is a quasiequivalence. Then we have an equivalence of categories $H^{0}(\mathcal{D}) \rightarrow H^{0}\left(\operatorname{Kar}^{d g}(\mathcal{D})\right)$. Since the latter category is idempotent complete, so is the former, i.e. $\mathcal{D}$ is homotopy idempotent complete.

We are ready to check the universal property of $\operatorname{Kar}^{d g}(\mathcal{C})$.

Proof of Theorem 4.3. Let $\mathcal{D}$ be a homotopy idempotent complete $\mathrm{dg}$ category, and let $F: \mathcal{C} \rightarrow \mathcal{D}$ be a dg functor. We extend $F$ to a dg functor $\operatorname{Tw}\left(\Sigma^{\Pi} \mathcal{C}\right) \rightarrow \operatorname{Tw}\left(\Sigma^{\Pi}\right)$; this restricts to a dg functor on the full subcategories $\operatorname{Kar}^{d g}(\mathcal{C}) \rightarrow \operatorname{Kar}^{d g}(\mathcal{D})$. Since $\mathcal{D}$ is homotopy idempotent complete, the canonical dg functor $\mathcal{D} \rightarrow \operatorname{Kar}^{d g}(\mathcal{D})$ is a quasi-equivalence by Lemma 4.20, and composing with the inverse (in Hqe) gives a quasi-functor $\widetilde{F}: \operatorname{Kar}^{d g}(\mathcal{C}) \rightarrow \mathcal{D}$ lifting $F$.

The uniqueness of this lift up to homotopy (again in Hqe) follows because if $e \in \operatorname{End}_{\mathcal{C}}(X)$ is a homotopy idempotent then $F(\operatorname{im} e)$ is determined uniquely up to homotopy by $F(X)$ and $F(e)$.

Lemma 4.21. Let $I$ be a finite poset and suppose we have objects $X_{i} \in \mathcal{C}$ equipped with homotopy idempotents $e_{i} \in \operatorname{End}_{\mathfrak{e}}^{0}(X)$. Suppose $Y_{i} \simeq \operatorname{im} e_{i}$ for $i \in I$. Then any one-sided twist $\operatorname{tw}_{\alpha}\left(\bigoplus_{i} Y_{i}\right)$ is the image of some homotopy idempotent $f$ acting on some one-sided twist $\operatorname{tw}_{\beta}\left(\bigoplus_{i} X_{i}\right)$.

Proof. It is sufficient to prove the statement for two-term twisted complexes, i.e. cones. Suppose that $Y_{0}, Y_{1}$ are homotopy summands of $X_{0}, X_{1}$ corresponding to homotopy idempotents $e_{0}, e_{1}$ and $g: Y_{0} \rightarrow Y_{1}$ is a closed degree zero morphism. There are inclusions $\sigma_{i}: Y_{i} \rightarrow X_{i}$, projections $\pi_{i}: X_{i} \rightarrow Y_{i}$ and homotopies $h_{i}: Y_{i} \rightarrow Y_{i}$ such that $\pi_{i} \sigma_{i}=\operatorname{Id}_{Y_{i}}+d\left(h_{i}\right)$. Define $g^{\prime}:=\sigma_{1} g \pi_{0}$, and consider the following chain maps between Cone $(g)$ and Cone $\left(g^{\prime}\right)$ :

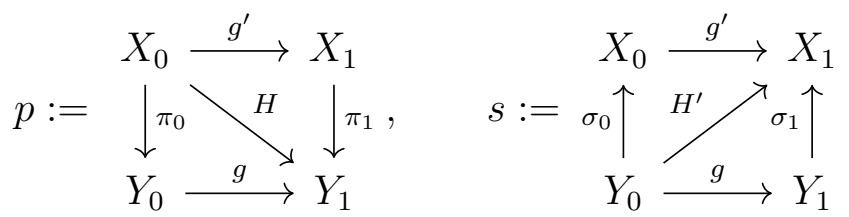

where $H=h_{1} g \pi_{0}$ and $H^{\prime}=\sigma_{1} g h_{0}$. It is easy to see that $p \circ s$ is homotopic to identity, so Cone $(g)$ is a homotopy summand of Cone $\left(g^{\prime}\right)$.

Theorem 4.22. If $\mathcal{C}$ is pretriangulated, then so is $\operatorname{Kar}^{d g}(\mathcal{C})$.

Proof. Every object of $\operatorname{Kar}^{d g}(\mathcal{C})$ is (isomorphic to) a homotopy summand of an object in $\mathrm{C}$. A one-sided twisted complex constructed from homotopy summands of $Y^{i} \in \mathcal{C}$ $(i \in I)$ is also a homotopy summand of a twisted complex constructed from $Y^{i}$ by Lemma 4.21; such objects live in $\operatorname{Kar}^{d g}(\mathcal{C})$ since $\mathcal{C}$ is pretriangulated and $\operatorname{Kar}^{d g}(\mathcal{C})$ is idempotent complete. 
Remark 4.23. All the above constructions naturally extend to $A_{\infty}$-categories, see [Sei08] for details. In particular, the equation for an $A_{\infty}$-idempotent in Proposition 4.15 should be replaced with

$$
\sum_{d} \sum_{i_{1}, \ldots, i_{d}} \pm \mu_{d}\left(h^{\left(i_{1}\right)}, \ldots, h^{\left(i_{d}\right)}\right)= \begin{cases}h^{(k-1)} & \text { if } k \text { is odd } \\ 0 & \text { if } k \text { is even }\end{cases}
$$

where the sum is over all partitions $i_{1}+\ldots+i_{d}=k-d+1$.

The analogue of Proposition 4.15 is [Sei08, Lemma 4.2] which states that any homotopy idempotent can be lifted to an $A_{\infty}$-idempotent. This allows one to define the Karoubi completion $\operatorname{Kar}(\mathcal{C})$ of an $A_{\infty}$-category $\mathcal{C}$, which is again an $A_{\infty}$-category and satisfies the universal property as above.

4.6. Perfect complexes are Karoubian. Let $A$ be a $\mathrm{dg}$ algebra. Throughout this section we will assume that $A$ is supported in non-positive cohomological degrees.

Let $\langle A\rangle$ to be the category of finitely generated free $A$-modules, that is, direct sums of finitely many copies of $A$. We define $\operatorname{Pretr}(A)$ to be the category of (finite) twisted complexes of free $A$-modules or, equivalently, the pretriangulated hull of $\langle A\rangle$. We define $\operatorname{Kar}^{d g}\langle A\rangle$ to be the category of projective $A$-modules, that is, homotopy direct summands of objects in $\langle A\rangle$. Let $\operatorname{Perf}(A)$ be the category of perfect twisted complexes over $\mathrm{A}$, that is, bounded twisted complexes built out of objects in $\operatorname{Kar}^{d g}\langle A\rangle$. In other words,

$$
\operatorname{Perf}(A)=\operatorname{Pretr}\left(\operatorname{Kar}^{d g}\langle A\rangle\right) .
$$

Finally, we define $\mathrm{Ch}^{b}(A)$ (resp. $\left.\mathrm{Ch}^{b}\left(\operatorname{Kar}^{d g}\langle A\rangle\right)\right)$ to be the category of bounded complexes of free (resp. projective) $A$-modules.

By definition, an object of $\operatorname{Pretr}(A)$ is a graded free $\mathrm{dg}$ module $M=\oplus_{i} \Sigma^{-i} M_{i}$ with $M_{i}$ in $\langle A\rangle$, and a differential given by a matrix $d=\left(d_{i j}\right)$ where $d_{i j}: M_{j} \rightarrow M_{i}$ and $d_{i i}$ agrees with the internal differential on $M_{i}$. The differential $d$ is homogeneous of cohomological degree 1 , so $d_{i j}$ has cohomological degree $1+j-i$. Since $A$ is non-positively graded, we have $d_{i j}=0$ unless $i \geq j$, so the matrix $d=\left(d_{i j}\right)$ is lower-triangular. Also, $d^{2}=0$ which means

$$
\sum_{k: i \geq k \geq j} d_{i k} d_{k j}=0 \text { for all } i>j
$$

Similarly, if $M=\left(\oplus_{i} \Sigma^{-i} M_{i}, d\right)$ and $N=\left(\oplus_{i} \Sigma^{-i} N_{i}, d\right)$ then a morphism from $M$ to $N$ is given by an lower-triangular matrix of morphisms $f=\left(f_{i j}\right)$ where $f_{i j}$ is a morphism from $M_{j}$ to $N_{i}$ of cohomological degree $j-i$.

This immediately implies the following:

Proposition 4.24. Suppose that the differential on $A$ vanishes and let $(M, d)$ be a twisted complex in $\operatorname{Pretr}(A)$, and $d^{\prime}=\sum_{i} d_{i+1, i}$. Then $\left(d^{\prime}\right)^{2}=0$, so $\left(M, d^{\prime}\right)$ is a well-defined chain complex in $\mathrm{Ch}^{b}(A)$.

Remark 4.25. A similar construction works if the differential on $A$ is non-trivial, but its component from $A_{-1}$ to $A_{0}$ vanishes. 
Remark 4.26. We can define a similar construction for an $A_{\infty}$-algebra $A$ with $\mu_{1}=0$. Indeed, the $A_{\infty}$-Maurer-Cartan equation for $d$ still implies $d_{i+2, i+1} d_{i+1, i}=0$.

We will call $\left(M, d^{\prime}\right)$ the underlying complex of the twisted complex $(M, d)$. This construction defines $\mathrm{dg}$ functors

$$
\epsilon: \operatorname{Pretr}(A) \rightarrow \mathrm{Ch}^{b}(A), \quad \epsilon: \operatorname{Perf}(A) \rightarrow \mathrm{Ch}^{b}\left(\operatorname{Kar}^{d g}\langle A\rangle\right)
$$

We will denote both of these functors by $\epsilon$ since it will be clear from the context which one is used.

The following lemma is a standard application of perturbation theory.

Lemma 4.27. Suppose that $X$ is an object in $\operatorname{Pretr}(A)$ or $\operatorname{Perf}(A)$. If $\epsilon(X)$ is contractible then $X$ is contractible. If $f: X \rightarrow Y$ is a morphism such that $\epsilon(f)$ is a homotopy equivalence, then $f$ is a homotopy equivalence.

Proof. For the first part see e.g [GH17, Lemma 2.19]. For the second part, observe that $f$ is a homotopy equivalence if and only if the cone of $f$ is contractible.

Theorem 4.28. Assume that $A$ is non-positively graded and the differential on $A$ vanishes. Then the category $\operatorname{Perf}(A)$ is homotopy idempotent complete, and

$$
\operatorname{Kar}^{d g}(\operatorname{Pretr}(A))=\operatorname{Perf}(A)
$$

Remark 4.29. The proof is similar to [BV08, Lemma 1.5.6(iii)] and [GW19, Appendix], but we include it here for completeness and add more details for reader's convenience.

Proof. Let $X$ be a twisted complex in $\operatorname{Perf}(A)$, and let $e$ be a homotopy idempotent endomorphism of $X$. Without loss of generality we can suppose that $X$ is a (one-sided) twist of

$$
\bigoplus_{0<i<(2 N-1)} \Sigma^{-i} X_{i}
$$

for some sufficiently large $N$.

As in Section 4.4, we can lift $e$ to an $A_{\infty}$ idempotent $(X, \underline{e}, \underline{h})$ and consider a twisted complex $Z$ built out of several copies of $X$ connected by the maps from $\underline{e}$ and $\underline{h}$ with appropriate signs. As in Remark 4.14 we build a bounded $Z$ from $2 N$ of copies of $X$, which is homotopy equivalent to the image of $e$ acting on $X \oplus \Sigma^{2 N-1} X$ via an argument similar to Lemma 4.12. By our assumptions, $X$ is a direct sum of copies of $\Sigma^{-i} A$ with $i>0$, and $\Sigma^{2 N-1} X$ is a direct sum of copies of $\Sigma^{-i} A$ with $i<0$. There are no nonzero $A$-module maps from $\Sigma^{-j} A \rightarrow \Sigma^{-i} A$ unless $i>j$, so we get the following diagram: 


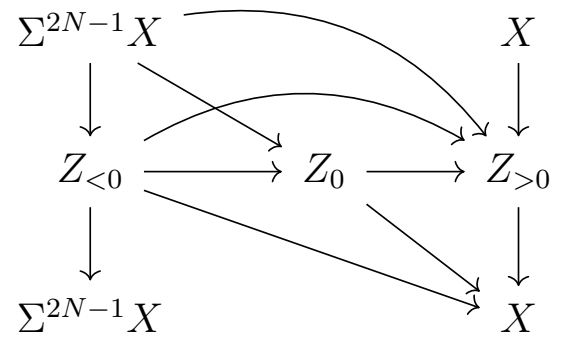

where the top half of the diagram represents the projection $\pi: X \oplus \Sigma^{2 N-1} X \rightarrow Z$ and the bottom half represents the inclusion $i: Z \rightarrow X \oplus \Sigma^{2 N-1} X$. We decomposed $Z=\bigoplus_{a} \Sigma^{i_{a}} A$ into its summands $Z_{>0}, Z_{0}, Z_{<0}$ corresponding to those indices $a$ for which $i_{a}>0, i_{a}=0$, or $i_{a}<0$, respectively (recall that $\Sigma$ is the suspension so in fact lowers cohomological degree).

Observe that $\pi \circ i$ is homotopic to identity on $Z$ and vanishes on $Z_{0}$. Since the differential on $A$ vanishes, by Proposition 4.24 one can define the forgetful $\mathrm{dg}$ functor $\epsilon: \operatorname{Perf}(A) \rightarrow \operatorname{Ch}\left(\operatorname{Kar}^{d g}(A)\right)$. By applying it to $Z$ we get a chain complex $\epsilon(Z)$ and a homotopy $h \in \operatorname{End}(\epsilon(Z))$ such that $d h+\left.h d\right|_{Z_{0}}=\operatorname{Id}_{Z_{0}}$. Now

$$
(d h)^{2}=(d h+h d) d h=d h,(h d)^{2}=h d(d h+h d)=h d,
$$

so $d h$ and $h d$ are two orthogonal idempotents on $Z_{0}$. Since $\operatorname{Kar}^{d g}\langle A\rangle$ is Karoubian, we can split $Z_{0}=Q \oplus Q^{\prime}$.

For the final step, let $T$ be the natural twisted complex built out of $Q$ and $Z_{>0}$ (abstractly speaking, this is the cone of the composition $\Sigma^{-1} Q \hookrightarrow \Sigma^{-1} Z_{0} \rightarrow Z_{>0}$ ). It is easy to see that $T$ is a subcomplex of $Z$ and we can restrict the maps $i$ and $\pi$ from the above diagram to $i^{\prime}: T \rightarrow X$ and $\pi^{\prime}: X \rightarrow T$. Then $i^{\prime} \circ \pi^{\prime}=\left.i \circ \pi\right|_{X} \simeq e$ and $\epsilon\left(\pi^{\prime} \circ i^{\prime}\right) \simeq \operatorname{Id}_{\epsilon(T)}$. By Lemma 4.27 we get that $\pi^{\prime} \circ i^{\prime}$ is homotopic to $\operatorname{Id}_{T}$. Therefore $T$ represents the image of $e$, and we conclude that $X$ is homotopy equivalent to a perfect $A$-module.

This shows that $\operatorname{Perf}(A)$ is homotopy Karoubian.

Suppose that a $\mathrm{dg}$ algebra $A$ retracts to its $\operatorname{dg}$ module $V$. Recall that in section 2.5 we defined $A_{\infty}$-operations $\mu_{k}: V^{\otimes k} \rightarrow V$.

Lemma 4.30. Define $\operatorname{Perf}(V)=\operatorname{Pretr}(\operatorname{Kar}\langle V\rangle)$ where $\langle V\rangle$ is the $A_{\infty}$-category with a single object with endomorphism $A_{\infty}$-algebra $V$. Then the $A_{\infty}$-categories $\operatorname{Perf}(A)$ and $\operatorname{Perf}(V)$ are quasi-equivalent.

Proof. We can consider $A$ and $V$ as $A_{\infty}$-categories $(*, A)$ and $(*, V)$ with one object with endomorphism algebra $A$ or $V$, respectively. We claim that $(*, A)$ and $(*, V)$ are quasi-equivalent.

Indeed, there exist $A_{\infty}$-algebra homomorphisms between $A$ and $V$ defined by $A_{\infty}$-maps $\lambda_{k}: V^{\otimes k} \rightarrow A$ from the proof of Theorem 2.10. By Theorem 2.10), these $A_{\infty}$-algebra homomorphisms induce quasi-isomorphisms on homology, so $(*, A)$ and $(*, V)$ are quasi-equivalent as $A_{\infty}$ categories. 
The corresponding categories of free $\mathrm{dg}$ modules $\langle A\rangle$ and $\langle V\rangle$ are nothing but additive closures of $(*, A)$ and $(*, V)$, so these are quasi-equivalent. Therefore $\operatorname{Kar}^{d g}\langle A\rangle$ and $\operatorname{Kar}^{d g}\langle V\rangle$ are quasi-equivalent by Theorem 4.3 and their pretriangulated hulls are quasi-equivalent as well.

\section{THE BAR COMPLEX FOR DG CATEGORIES}

One can motivate the introduction of the (two-sided) bar complex of a dg category as the object which governs the notion of "naturality up to coherent homotopy". To illustrate, let $\mathcal{C}$ and $\mathcal{D}$ be $\mathrm{dg}$ categories and $F, G: \mathcal{C} \rightarrow \mathcal{D} \mathrm{dg}$ functors. We have already introduced the notion of a (say, degree zero) natural transformation, which is a choice of morphism $\alpha_{X} \in \operatorname{Hom}_{\mathcal{D}}(F(X), G(X))$ for all $X \in \mathcal{C}$, natural with respect to morphisms in $\mathcal{C}$. This means for every morphism $f: X \rightarrow Y$ in $\mathcal{C}$ we have

$$
G(f) \circ \alpha_{X}-\alpha_{Y} \circ F(f)=0 .
$$

Said differently, the pair of $\mathrm{dg}$ functors $F, G$ determines a $\mathcal{C}, \mathcal{C}$-bimodule $\mathcal{B}(G, F)$, which for $X, Y \in \mathcal{C}$ has

$$
Y \mathcal{B}(G, F) X:=G(Y) \mathcal{D} F(X),
$$

(in the notation of $\$ 2.4$ ) and a natural transformation is simply a map of $\mathcal{C}, \mathcal{C}$-bimodules

$$
\alpha: \mathcal{C} \rightarrow \mathcal{B}(G, F) .
$$

The image of $\operatorname{Id}_{X} \in \mathcal{C}$ is the chosen morphism $\alpha_{X}$, and naturality is equivalent to $\alpha$ being a $\mathcal{C}, \mathcal{C}$-bimodule map.

Now suppose that we are in a situation where naturality does not hold on the nose, but only up to homotopies $\alpha_{f} \in \operatorname{Hom}_{\mathcal{D}}^{-1}(F(X), G(Y))$ :

$$
d_{\mathcal{D}}\left(\alpha_{f}\right)=G(f) \circ \alpha_{X}-\alpha_{Y} \circ F(f) .
$$

Let us consider another morphism $g: Y \rightarrow Z$ in $\mathcal{C}$ and suppose that $f$ and $g$ are closed. It is straightforward to check that the expression

$$
\alpha_{g \circ f}-G(g) \circ \alpha_{f}-\alpha_{g} \circ F(f)
$$

is closed. It is often desirable to suppose this expression is also exact, i.e. there exists a homotopy $\alpha_{g, f} \in \operatorname{Hom}_{\mathcal{D}}^{-2}(F(X), G(Z))$. Roughly speaking, this says that the assignment $f \mapsto \alpha_{f}$ satisfies a version of the Leibniz rule, up to homotopy.

Now, assuming the existence of such "higher homotopies" $\alpha_{f, g}$ for all composable morphisms $f, g$ one can then define a degree -3 closed morphism associated to each triple of composable morphisms $f_{1}, f_{2}, f_{3}$, the exactness of which would allow us to define a family of closed morphisms (obstructions) associated to each 4-tuple of composable morphisms, and so on.

If all such obstructions are exact (and a family of homotopies realizing this exactness is given) then then we say that the system $\left(\alpha_{X}, \alpha_{f}, \alpha_{g, f}, \cdots\right)$ is a homotopy coherent natural transformation.

The two-sided bar complex $\mathrm{B}(\mathcal{C})$ of $\mathcal{C}$, which we describe explicitly below, can be considered as a free resolution of the trivial $\mathcal{C}, \mathcal{C}$-bimodule $\mathcal{C}$, and is spanned as a 
bimodule by sequences of composable morphisms of arbitrary finite length $r \geq 0$. The data of a homotopy coherent natural transformation $F \rightarrow G$ is then encoded as a map of $\mathcal{C}, \mathcal{C}$-modules

$$
\mathbf{B}(\mathcal{C}) \rightarrow \mathcal{B}(G, F) .
$$

Various operations on natural transformations can be understood in terms of various structures on the two-sided bar complex; for instance the composition of natural transformations can be understood via a natural comultiplication on $\mathrm{B}(\mathrm{C})$.

After this motivation, we now give an explicit description of the two-sided bar complex.

5.1. The two-sided bar complex of a pair. Let $\mathcal{C}$ be a dg category and $\mathcal{J} \subset \mathcal{C}$ a (unital) subcategory. We wish to define the two-sided bar complex associated to $(\mathcal{J}, \mathcal{C})$. First, consider $\mathcal{C}, \mathcal{C}$-bimodules of the form $\mathcal{C} \otimes_{\mathcal{J}} \cdots \otimes_{\mathfrak{J}} \mathcal{C}$.

Example 5.1. In case $\mathcal{J}=\mathcal{J}_{\mathcal{C}}$ is the subcategory of identity maps from Example 2.4, $\mathcal{C} \otimes_{\mathfrak{J}} \cdots \otimes_{\mathfrak{J}} \mathcal{C}$ is spanned by composable morphisms in $\mathcal{C}$. More generally, if $\mathcal{B} \subset \mathcal{C}$ is a full subcategory and $\mathcal{J}=\mathcal{J}_{\mathcal{B}} \subset \mathcal{C}$ is the subcategory of identity morphisms in $\mathcal{B}$, then

$$
\underbrace{\mathcal{C} \otimes_{\mathcal{J}} \otimes \cdots \otimes_{\mathcal{J}} \mathcal{C}}_{r+2 \text { factors }}
$$

is spanned as a bimodule by sequences of composable morphisms, which we denote by $f_{0}\left\|f_{1}\right\| \cdots \| f_{r+1}$, in which $f_{1}, \ldots, f_{r}$ are morphisms in the full subcategory $\mathcal{B} \subset \mathcal{C}$.

The two-sided bar complex associated to the pair $\mathcal{J} \subset \mathcal{C}$ is by definition the total complex $\mathrm{B}(\mathcal{C} ; \mathcal{J})$ of the following bicomplex:

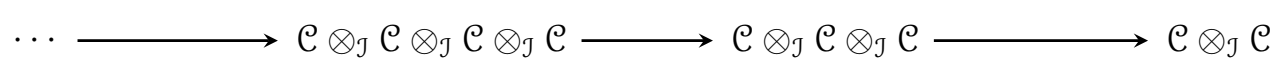

in which the horizontal arrows are

$$
f_{0}\|\cdots\| f_{r+1} \mapsto \sum_{i=0}^{r}(-1)^{i} f_{0}\|\cdots\| f_{i} \circ f_{i+1}\|\cdots\| f_{r+1} .
$$

More precisely,

$$
\mathbf{B}(\mathrm{C} ; \mathcal{J})=\bigoplus_{r \geq 0} \bigoplus_{X_{1}, \ldots, X_{r+1}} \Sigma^{r}\left(\mathcal{C} X_{1} \otimes X_{1} \mathcal{e} X_{2} \otimes \cdots \otimes X_{r+1} \mathrm{e}\right)
$$

where the direct sum is over objects $X_{1}, \ldots, X_{r+1}$ for which $\operatorname{Id}_{X_{i}} \in \mathcal{J}$ for $i=1, \ldots, r+1$. The differential on the two-sided bar complex is $d_{\oplus}+d_{\mathrm{bar}}$, where $d_{\oplus}$ is the direct sum of differentials on the terms above (inherited from $d_{\mathfrak{e}}$ by the usual tensor product rule, and a sign $(-1)^{r}$ coming from the $r$-translation), and $d_{\text {bar }}$ is defined by

$$
d_{\mathrm{bar}}\left(f_{0}\|\cdots\| f_{r+1}\right)=\sum_{i=0}^{r}(-1)^{i} f_{0}\|\cdots\| f_{i} \circ f_{i+1}\|\cdots\| f_{r+1} .
$$


(The notation is suggestive: the bar differential is the alternating sum of erasing bars.) The bimodule structure is given term-wise by

$$
g \cdot\left(f_{0}\left\|f_{1}\right\| \cdots\left\|f_{r}\right\| f_{r+1}\right) \cdot g^{\prime}:=(-1)^{|g| r}\left(g \circ f_{0}\right)\left\|f_{1}\right\| \cdots\left\|f_{r}\right\|\left(f_{r+1} \circ g^{\prime}\right) .
$$

Note that the left-action of bimodules obtains a sign-twist under translation.

By convention $\mathbf{B}(\mathcal{C})$ denotes $\mathbf{B}(\mathcal{C}, \mathcal{J})$ where $\mathcal{J}=\mathcal{J}_{\mathcal{C}}$ is the subcategory of identity maps in $\mathrm{C}$.

Remark 5.2. At the beginning of this section we have motivated the bar complex by claiming that $\mathcal{C}$, $\mathcal{C}$-bimodule maps $\alpha: \mathbf{B}(\mathcal{C}) \rightarrow \mathcal{B}(G, F)$ encode homotopy coherent natural transformations between $\mathrm{dg}$ functors $F, G: \mathcal{C} \rightarrow \mathcal{D}$. Indeed, the data of such a bimodule map is precisely the choice of closed degree zero morphisms $\alpha_{X}:=\alpha\left(\operatorname{Id}_{X} \| \operatorname{Id}_{X}\right)$ from $F(X)$ to $G(X)$ in $\mathcal{D}$, together with morphisms $\alpha\left(\operatorname{Id}_{Y}\|f\| \operatorname{Id}_{X}\right)$ which realize naturality up to homotopy, and the higher homotopies $\alpha\left(\operatorname{Id}_{X_{0}}\left\|f_{1}, \ldots, f_{r}\right\| \operatorname{Id}_{X_{r+1}}\right)$ which provide the required higher naturality data.

Proposition 5.3. The two-sided bar complex $\mathbf{B}(\mathcal{C}, \mathcal{J})$ is a coalgebra object in the category of $\mathcal{C}, \mathcal{C}$-bimodules: we have maps of bimodules $\mathbf{B}(\mathcal{C}, \mathcal{J}) \rightarrow \mathcal{C}$ and $\mathbf{B}(\mathcal{C}, \mathcal{J}) \rightarrow \mathbf{B}(\mathcal{C}, \mathcal{J}) \otimes_{\mathfrak{e}} \mathbf{B}(\mathcal{C}, \mathcal{J})$ satisfying the usual counit and coassociativity relations.

Proof. The counit $\varepsilon: \mathbf{B}(\mathcal{C}, \mathcal{J}) \rightarrow \mathcal{C}$ is defined componentwise by

$$
f_{0}\|\cdots\| f_{r+1} \mapsto\left\{\begin{array}{ll}
f_{0} \circ f_{1} & \text { if } r=0 \\
0 & \text { otherwise }
\end{array} .\right.
$$

The comultiplication $\Delta: \mathbf{B}(\mathcal{C}, \mathcal{J}) \rightarrow \mathbf{B}(\mathcal{C}, \mathcal{J}) \otimes_{\mathfrak{C}} \mathbf{B}(\mathcal{C}, \mathcal{J})$ is defined componentwise by

$$
f_{0}\|\cdots\| f_{r+1} \mapsto \sum_{i=1}^{r+1}(-1)^{(r-i+1)\left(\left|f_{0}\right|+\cdots+\left|f_{i-1}\right|\right)}\left(f_{0}\|\cdots\| f_{i-1} \| \mathrm{Id}\right) \otimes\left(\operatorname{Id}\left\|f_{i}\right\| \cdots \| f_{r+1}\right) .
$$

The counit and coassociativity axioms are easily checked. Furthermore, it is clear that $\Delta$ and $\varepsilon$ are closed and commute with the $\mathcal{C}, \mathcal{C}$-bimodule structure on $\mathbf{B}(\mathcal{C}, \mathcal{J})$.

Let us give an alternate description of the two-sided bar complex. The bimodule $\mathcal{C} \otimes_{\mathfrak{J}} \mathcal{C}$ is a coalgebra object $C_{\mathfrak{J}}$ in $\mathcal{C}, \mathcal{C}$-bimodules, and $\mathbf{B}(\mathcal{C}, \mathcal{J})$ is the counital idempotent $\mathbf{P}_{C_{J}}$ associated to $C_{\mathcal{J}}[\operatorname{Hog} 17]$.

If $\mathcal{J} \subset \mathcal{J} \subset \mathcal{Q}$, then the counit of $C_{\mathfrak{J}}$ factors through the counit of $C_{\mathfrak{g}}$, so $C_{\mathfrak{J}} \leq C_{\mathfrak{J}}$ in the notation of [Hog17]. The following is an immediate consequence of this.

Proposition 5.4. If $\mathcal{J} \subset \mathcal{J} \subset \mathcal{C}$ are unital subcategories then

$$
\mathbf{B}(\mathcal{C}, \mathcal{J}) \otimes_{\mathfrak{e}} \mathbf{B}(\mathcal{C}, \mathcal{J}) \simeq \mathbf{B}(\mathcal{C}, \mathcal{J}) \simeq \mathbf{B}(\mathcal{C}, \mathcal{J}) \otimes_{\mathfrak{e}} \mathbf{B}(\mathcal{C}, \mathcal{J}) .
$$

\subsection{Shrinking the bar complex.}

Definition 5.5. We say that a full subcategory $\mathcal{B} \subset \mathcal{C}$ generates $\mathcal{C}$ if for all $\mathrm{dg}$ functors $F: \mathcal{C} \rightarrow \mathbb{k}$-dgmod, $F(X) \simeq 0$ for all $X \in \mathcal{B}$ implies $F(X) \simeq 0$ for all $X \in \mathcal{C}$. 
Proposition 5.6. Suppose every object of $\mathcal{C}$ is homotopy equivalent to a finite one-sided twisted complex constructed from objects of $\mathcal{B}$. Then $\mathcal{B}$ generates $\mathcal{C}$ in the sense of Definition 5.5 .

Proof. Let $F: \mathcal{C} \rightarrow \mathbb{k}$-dgmod be a dg functor such that $F(X) \simeq 0$ for all $X \in \mathcal{B}$, and let $Z=\operatorname{tw}_{\delta}\left(\bigoplus_{i} \Sigma^{a_{i}} X_{i}\right)$ be a finite one-sided twisted complex. Then $F(Z)$ is isomorphic to a one-sided twisted complex $\operatorname{tw}_{F(\delta)}\left(\bigoplus_{i} \Sigma^{a_{i}} F\left(X_{i}\right)\right)$. It follows that $F(Z) \simeq 0$ since finite one-sided twisted complexes built from contractible complexes are contractible.

Remark 5.7. The statement of Proposition 5.6 remains true if we replace "finite" with "bounded above".

Proposition 5.8. If $\mathcal{B} \subset \mathcal{C}$ generates, then the natural inclusion $\mathbf{B}\left(\mathcal{C}, \mathcal{J}_{\mathcal{B}}\right) \rightarrow \mathbf{B}\left(\mathcal{C}, \mathcal{J}_{\mathcal{C}}\right)$ is the section of a deformation retract.

Proof. Let $\mathcal{J}=\mathcal{J}_{\mathcal{C}} \subset \mathcal{C}$ be the subcategory of identity morphisms in $\mathcal{C}$, and let $\mathcal{J}=\mathcal{J}_{\mathcal{B}} \subset$ $\mathcal{C}$ be the subcategory of identity morphisms in $\mathcal{B}$. Let $C=\mathcal{C} \otimes_{\mathfrak{J}} \mathcal{C}$ and $D=\mathcal{C} \otimes_{\mathfrak{j}} \mathrm{e}$.

Note that $C=D \oplus E$, where $E=\bigoplus_{Y \notin \mathcal{B}} \mathrm{C} Y \otimes Y \mathcal{C}$.

Consider the $\mathcal{C}$, $\mathcal{C}$-bimodules $\mathbf{P}_{C}, \mathbf{P}_{D}$ and $\mathbf{A}_{D}=\operatorname{Cone}\left(\mathbf{P}_{D} \rightarrow \mathcal{C}\right)$. Since $C=D \oplus E$, it follows that $\mathbf{P}_{C}$ can be expressed as a one-sided twisted complex constructed from one copy of $\mathbf{P}_{D}$ and terms of the form

$$
\mathbf{A}_{D} \otimes_{\mathfrak{e}} E \otimes_{\mathfrak{e}} \mathbf{A}_{D} \otimes_{\mathfrak{e}} \cdots \otimes_{\mathfrak{e}} E \otimes_{\mathfrak{e}} \mathbf{A}_{D}
$$

But one of the features of $\mathbf{A}_{D}$ is $Y \mathbf{A}_{D} \simeq 0 \simeq \mathbf{A}_{D} Y$ for all $Y \in \mathcal{B}$. Since $\mathcal{B}$ generates, it follows that $Y \mathbf{A}_{D} \simeq 0 \simeq \mathbf{A}_{D} Y$ for all $Y \in \mathcal{C}$. In particular $E \otimes_{\mathcal{e}} \mathbf{A}_{D} \simeq 0 \simeq \mathbf{A}_{D} \otimes_{\mathcal{e}} E$. Contracting the contractible terms yields the desired deformation retract

$$
\mathbf{P}_{C}=\mathbf{P}_{D \oplus E} \rightarrow \mathbf{P}_{D}
$$

5.3. The bar complex of the pretriangulated hull. Let $\mathcal{C}$ be a dg category and $\operatorname{Pretr}(\mathcal{C})$ its pretriangulated hull. We have a homotopy equivalence of bimodules

$$
\mathbf{B}\left(\operatorname{Pretr}(\mathcal{C}), \mathcal{J}_{\operatorname{Pretr}(\mathcal{C})}\right) \rightarrow \mathbf{B}\left(\operatorname{Pretr}(\mathcal{C}), \mathcal{J}_{\mathcal{C}}\right)
$$

implicitly constructed in the previous section. It will be useful to understand this map explicitly.

We first consider the relation between the two-sided bar complexes of $\mathcal{C}$ and $\Sigma \mathcal{C}$. There is a map of $\Sigma \mathcal{C}, \Sigma \mathcal{C}$-bimodules

$$
\Phi: \mathbf{B}\left(\Sigma \mathcal{C}, \mathcal{J}_{\Sigma \mathcal{e}}\right) \rightarrow \mathbf{B}\left(\Sigma \mathcal{C}, \mathcal{J}_{\mathfrak{C}}\right)
$$

defined as follows: given objects $X_{0}, \ldots, X_{r} \in \Sigma \mathcal{C}$ of the form

$$
X_{i}=\bigoplus_{j} \Sigma^{a_{i, j}} X_{i j}
$$

and a sequence of composable morphisms

$$
g_{1} \otimes \cdots \otimes g_{r} \in X_{0}(\Sigma \mathcal{C}) X_{1} \otimes \cdots \otimes X_{r-1}(\Sigma \mathcal{C}) X_{r},
$$


we define $\Phi\left(\operatorname{Id}\left\|g_{1}\right\| \cdots\left\|g_{r}\right\| \mathrm{Id}\right)$ to be the sum of terms of the form

$$
\pm \sigma_{j_{0}}\left\|\left(g_{1}\right)_{j_{0}, j_{1}}\right\|\left(g_{2}\right)_{j_{1}, j_{2}}\|\cdots\|\left(g_{r}\right)_{j_{r-1}, j_{r}} \| \pi_{j_{r}},
$$

where $\left(g_{i}\right)_{j_{i-1}, j_{i}}$ indicates the component of $g_{i}$ living in $X_{i-1, j_{i-1}} \mathrm{e} X_{i, j_{i}}$ and $\sigma_{j_{0}}$ denotes the inclusion of $X_{0, j_{0}}$ into $X_{0}$ and $\pi_{j_{r}}$ denotes the projection of $X_{r}$ onto $X_{r, j_{r}}$. It is an exercise to find the correct signs such that $\Phi$ defines a deformation retract.

Next we consider the relation between the two-sided bar complex of $\mathcal{C}$ and $\operatorname{Tw}(\mathcal{C})$. We define a map of $\mathcal{C}, \mathcal{C}$-bimodules

$$
\Psi: \mathbf{B}\left(\operatorname{Tw}(\mathcal{C}), \mathcal{J}_{\mathrm{Tw}(\mathcal{C})}\right) \rightarrow \mathbf{B}\left(\operatorname{Tw}(\mathcal{C}), \mathcal{J}_{\mathcal{C}}\right)
$$

as follows. Given objects $\operatorname{tw}_{\alpha_{i}}\left(X_{i}\right) \in \operatorname{Tw}(\mathcal{C})(0 \leq i \leq r)$ and an element

$$
\operatorname{Id}\left\|f_{1}\right\| \cdots\left\|f_{r}\right\| \operatorname{Id} \in \mathbf{B}\left(\operatorname{Tw}(\mathcal{C}), \mathcal{J}_{\mathrm{Tw}(\mathcal{C})}\right),
$$

we define $\Psi\left(\operatorname{Id}\left\|f_{1}\right\| \cdots\left\|f_{r}\right\| \operatorname{Id}\right)$ to be the sum of terms of the form

$$
\pm \phi\|\underbrace{\alpha_{0}\|\cdots\| \alpha_{0}}_{i_{0}}\| f_{1}\|\underbrace{\alpha_{1}\|\cdots\| \alpha_{1}}_{i_{1}}\| \cdots\|\underbrace{\alpha_{r-1}\|\cdots\| \alpha_{r-1}}_{i_{r-1}}\| f_{r}\|\underbrace{\alpha_{r}\|\cdots\| \alpha_{r}}_{i_{r}}\| \phi^{-1}
$$

where $i_{0}, \ldots, i_{r} \geq 0$ and $\phi$ and $\psi$ denote $\operatorname{Id}_{X_{0}}$ and $\operatorname{Id}_{X_{r}}$, regarded as degree zero (not necessarily closed) maps $X_{0} \rightarrow \mathrm{tw}_{\alpha_{0}}\left(X_{0}\right)$ and $X_{r} \rightarrow \mathrm{tw}_{\alpha_{r}}\left(X_{r}\right)$. It is an exercise to find the correct signs and check that this defines a deformation retract.

Combining these gives a bimodule map relating the two-sided bar complex of $\mathcal{C}$ and its pretriangulated hull; this map is a deformation retract and, in particular, a homotopy equivalence.

\subsection{The semi-orthogonal bar complex.}

Definition 5.9. Let $\Gamma$ be a poset, and let $\mathcal{B}_{\gamma} \subset \mathcal{C}$ be full $\mathrm{dg}$ subcategories of $\mathcal{C}$, indexed by $\gamma \in \Gamma$ such that

(1) every object $Z \in \mathcal{C}$ is homotopy equivalent to a one-sided twisted complex $\operatorname{tw}_{\delta}\left(\bigoplus_{\gamma \in \Gamma} Z_{\gamma}\right)$ where $Z_{\gamma} \in \mathcal{B}_{\gamma}$, only finitely many $Z_{\gamma}$ are nonzero and the twist $\delta$ is strictly lower triangular with respect to the partial order on $\Gamma$.

(2) $\operatorname{Hom}_{\mathcal{C}}\left(Y_{\gamma}, Y_{\gamma^{\prime}}\right)$ is contractible unless $\gamma \leq \gamma^{\prime}$.

Then we say that $\mathcal{B}_{\gamma}$ defines a $\Gamma$-indexed semi-orthogonal decomposition of $\mathcal{C}$.

Note that if $X \in \mathcal{B}_{\gamma} \cap \mathcal{B}_{\gamma^{\prime}}$ for $\gamma \neq \gamma^{\prime}$ then $X$ is contractible. Thus, we usually assume, without loss of generality, that the subcategories $\mathcal{B}_{\gamma}$ have no objects in common.

Let $\left\{\mathcal{B}_{\gamma}\right\}_{\gamma \in \Gamma}$ be a semi-orthogonal decomposition of $\mathcal{C}$. Define the full $\mathrm{dg}$ subcategory $\mathcal{B}:=\bigcup_{\gamma \in \Gamma} \mathcal{B}_{\gamma} \subset \mathcal{C}$, which generates $\mathcal{C}$ by Proposition 5.6.

For $X, Y \in \mathcal{B}$ we write $X \leq Y$ if $X \in \mathcal{B}_{\gamma}$ and $Y \in \mathcal{B}_{\gamma^{\prime}}$ with $\gamma \leq \gamma^{\prime}$. This relation is transitive and reflexive, but not anti-symmetric: $X \leq Y$ and $Y \leq X$ holds if and only if $X, Y \in \mathcal{B}_{\gamma}$ for some $\gamma$.

The semi-orthogonal two-sided bar complex of $\mathrm{C}$ is defined to be the subcomplex $\mathbf{B}^{\Gamma}\left(\mathcal{C}, \mathcal{J}_{\mathcal{B}}\right) \subset \mathbf{B}\left(\mathcal{C}, \mathcal{J}_{\mathcal{B}}\right)$ spanned by elements of the form

$$
f_{0}\|\cdots\| f_{r+1} \text { in } \mathrm{e} X_{1} \otimes X_{1} \mathrm{e} X_{2} \otimes \cdots \otimes X_{r+1} \mathrm{e}
$$


with $X_{1} \geq X_{2} \geq \cdots \geq X_{r+1}$.

Proposition 5.10. Retain notation as above. The inclusion $\mathbf{B}^{\Gamma}\left(\mathcal{C}, \mathcal{J}_{\mathcal{B}}\right) \hookrightarrow \mathbf{B}\left(\mathcal{C}, \mathcal{J}_{\mathcal{B}}\right)$ is the section of a deformation retract; the data of this deformation retract are $\mathrm{C}, \mathrm{C}$-bilinear.

Proof. The complex $\mathbf{B}\left(\mathcal{C}, \mathcal{J}_{\mathcal{B}}\right)$ is a one-sided twist of

$$
\bigoplus_{r \geq 0} \bigoplus_{X_{1}, \ldots, X_{r+1} \in \mathcal{B}} \Sigma^{r}\left(\mathrm{e} X_{1} \otimes_{\mathbb{k}} X_{1} \mathrm{e} X_{2} \otimes_{\mathbb{k}} \cdots \otimes_{\mathbb{k}} X_{r+1} \mathrm{e}\right)
$$

But $X_{i} \mathfrak{e} X_{i+1}=\operatorname{Hom}_{\mathfrak{e}}\left(X_{i+1}, X_{i}\right)$ is contractible unless $X_{i} \geq X_{i+1}$. Contracting all such terms gives the desired deformation retract. Since the contractions are all of the form

$$
\mathcal{C} X_{1} \otimes_{\mathbb{k}} \underbrace{X_{1} \mathcal{B} X_{2} \otimes_{\mathbb{k}} \cdots \otimes_{\mathbb{k}} X_{r} \mathcal{B} X_{r+1}}_{\text {contract some factor }} \otimes X_{r+1} \mathrm{e},
$$

(i.e. we only ever contract an "internal" tensor factor) the data of the deformation retract commute with the left and right $\mathrm{C}$-actions.

Since $\mathcal{B}$ generates $\mathcal{C}$, Proposition 5.8 implies the following.

Theorem 5.11. Retain notation as above. The full bar complex $\mathbf{B}(\mathcal{C})$ deformation retracts onto the semi-orthogonal bar complex $\mathbf{B}^{\Gamma}\left(\mathcal{C}, \mathfrak{J}_{\mathcal{B}}\right)$. The data of this deformation retract are e, e-bilinear.

Corollary 5.12. The semi-orthogonal bar complex $\mathrm{B}^{\Gamma}\left(\mathcal{C}, \mathcal{J}_{\mathcal{B}}\right)$ has the structure of an $A_{\infty}-$ algebra object in $\mathrm{C}, \mathrm{C}-$ bimodules, c.f. Remark 2.12.

5.5. Hochschild homology (vertical trace). If $\mathcal{A}$ is an additive $\mathbb{k}$-category then the (vertical) trace of $\mathcal{A}$ is the $\mathbb{k}$-module

$$
\mathrm{HH}_{0}(\mathcal{A}):=\bigoplus_{X \in \mathcal{A}} \operatorname{End}(X) / \operatorname{span}_{\mathbb{k}}\left\{g_{0} \circ g_{1}-g_{1} \circ g_{0}\right\}
$$

where $\left(g_{0}, g_{1}\right)$ runs over pairs of morphisms which are composable in either order.

Notation 5.13. The class of $f \in \operatorname{End}(X)$ in $\operatorname{HH}_{0}(\mathcal{A})$ will be denoted $[f]$. We also write $[X]:=\left[\operatorname{Id}_{X}\right]$ for the associated class in $\mathrm{HH}_{0}(\mathcal{A})$.

Remark 5.14. If $\mathcal{A}$ is monoidal, then $\mathrm{HH}_{0}(\mathcal{A})$ inherits the structure of an algebra via $[f] \cdot[g]:=[f \otimes g]$.

It is natural to consider a derived version of the vertical trace, in which the relation $g_{0} \circ g_{1}=g_{1} \circ g_{0}$ is not strictly enforced, but is achieved by the formal adjunction of a homotopy $h\left(g_{0}, g_{1}\right)$. In this setting it is also natural to allow our additive category to be an arbitrary dg category $\mathrm{C}$. After adjoining such homotopies, symbols of the form $h\left(g_{0} g_{1}, g_{2}\right)-h\left(g_{0}, g_{1} g_{2}\right)+h\left(g_{2} g_{0}, g_{1}\right)$ are automatically closed. In order avoid creating such new closed elements, one is forced to adjoin higher homotopies $h\left(g_{0}, g_{1}, g_{2}\right)$. Continuing in this fashion, one obtains a complex

$$
\mathbf{C}(\mathrm{e})=\bigoplus_{r \geq 0} \bigoplus_{X_{1}, \ldots, X_{r+1} \in \mathcal{C}} \Sigma^{r}\left(X_{1} \mathrm{e} X_{2} \otimes \cdots \otimes X_{r+1} \mathrm{e} X_{1}\right)
$$


where the direct sum is over objects $X_{1}, \ldots, X_{r+1} \in \mathcal{C}$. We write $\left\|f_{1}\right\| f_{2}\|\cdots\| f_{r+1}$ with $f_{i} \in X_{i} \mathrm{e} X_{i+1}$ (with indices taken modulo $r+1$ ) for elementary tensors in the degree $r$ part of (11).

The differential of such an element is

$$
\begin{aligned}
& d_{\mathbf{C}(\mathcal{C})}\left(\left\|f_{1}\right\| f_{2}\|\cdots\| f_{r+1}\right):=(-1)^{r} \sum_{i=1}^{r+1}(-1)^{\left|f_{1}\right|+\cdots+\left|f_{i-1}\right|}\left\|f_{1}\right\| \cdots\left\|d_{\mathfrak{C}}\left(f_{i}\right)\right\| \cdots \| f_{r+1} \\
& +\sum_{i=1}^{r}(-1)^{i}\left\|f_{1}\right\| \cdots\left\|f_{i} f_{i+1}\right\| \cdots\left\|f_{r+1}+(-1)^{\left|f_{1}\right|\left(r-1+\left|f_{2}\right|+\cdots+\left|f_{r+1}\right|\right)}\right\| f_{2}\|\cdots\| f_{r+1} f_{1} .
\end{aligned}
$$

Note that the differential $d_{\mathbf{C}}$ splits as $d_{\oplus}+\delta$ where $d_{\oplus}$ is the direct sum of differentials on the terms in (11), shown in the first line, while $\delta$ is an additional contribution shown in the second line.

The complex just constructed is called the cyclic bar complex of $\mathcal{C}$.

Definition 5.15. The cyclic bar complex of $\mathcal{C}$ (relative to a unital subcategory $\mathcal{J} \subset \mathcal{C}$ ) is the $\mathrm{dg} \mathbb{k}$-module $\mathrm{C}(\mathcal{C}, \mathcal{J})$ obtained as the quotient of the two-sided bar complex $\mathbf{B}(\mathcal{C}, \mathcal{J})$ modulo the $\mathbb{k}$-span $[\mathcal{C}, \mathbf{B}(\mathcal{C})]$ of elements of the form $f \cdot m-(-1)^{|m||f|} m \cdot f$ for all $m \in \mathbf{B}(\mathcal{C}, \mathcal{J})$ and all morphisms $f$ in $\mathcal{C}$.

The Hochschild homology $\mathrm{HH}$. $(\mathcal{C}, \mathcal{J})$ is defined to be the homology of $\mathbf{C}(\mathcal{C}, \mathcal{J})$. Hochschild homology is written with homological convention for gradings, and so we write $\mathrm{HH}_{k}(\mathcal{C}, \mathcal{J}):=H^{-k}(\mathbf{C}(\mathcal{C}, \mathcal{J}))$ for $k \geq 0$.

Remark 5.16. Instead of (11), the definition describes the cyclic bar complex as:

$$
\mathbf{C}(\mathcal{C})=\bigoplus_{r \geq 0} \bigoplus_{X_{0}, X_{1}, \ldots, X_{r+1} \in \mathcal{C}} \Sigma^{r}\left(X_{0} \mathrm{e} X_{1} \otimes X_{1} \mathrm{e} X_{2} \otimes \cdots \otimes X_{r+1} \mathrm{e} X_{0}\right) / \sim
$$

where the linear relation $\sim$ is generated by

$$
(-1)^{\left|f_{0}\right|\left(r+\left|f_{1}\right|+\cdots+\left|f_{r+1}\right|\right)} f_{0}\left\|f_{1}\right\| f_{2}\|\cdots\| f_{r+1} \sim \operatorname{Id}\left\|f_{1}\right\| f_{2}\|\cdots\| f_{r+1} f_{0}
$$

The previously introduced notation for elements of the cyclic bar complex simply drops the leading identity term after such a rewrite-in the example above: $\left\|f_{1}\right\| f_{2}\|\cdots\| f_{r+1} f_{0}$.

Remark 5.17. When $\mathcal{J}=\mathcal{J}_{\mathrm{e}}$ is the subcategory of identity morphisms, then we have $\mathbf{C}(\mathcal{C}, \mathcal{J})=\mathbf{C}(\mathcal{C})$ as in (11). If $\mathcal{B} \subset \mathcal{C}$ is a full subcategory and $\mathcal{J}=\mathcal{J}_{\mathcal{B}}$ is the subcategory of identity morphisms in $\mathcal{B}$, then $\mathbf{C}\left(\mathcal{C}, \mathcal{J}_{\mathcal{B}}\right)=\mathbf{C}(\mathcal{B})$.

Remark 5.18. If $\mathcal{C}$ has trivial grading and trivial differential then $\mathrm{HH}_{0}(\mathcal{C})$ coincides with the vertical trace defined earlier.

An inclusion of subcategories $\mathcal{J} \subset \mathcal{J} \subset \mathcal{C}$ gives a chain map $\mathbf{C}(\mathcal{C}, \mathcal{J}) \rightarrow \mathbf{C}(\mathcal{C}, \mathcal{J})$. In particular, if $\mathcal{B} \subset \mathcal{C}$ is a full subcategory then we have a canonical inclusion $\mathrm{C}(\mathcal{B}) \hookrightarrow \mathrm{C}(\mathrm{C})$.

The following is our main tool for computing HH. (e). 
Theorem 5.19. If $\mathcal{B} \subset \mathcal{C}$ generates $\mathcal{C}$ (see Definition 5.5) then the natural inclusion $\mathbf{C}(\mathcal{B}) \hookrightarrow$ $\mathrm{C}(\mathrm{C})$ is the section of a deformation retract. More generally, if $\Gamma$ is a finite poset and $\left\{\mathcal{B}_{\gamma}\right\}_{\gamma \in \Gamma}$ gives a semi-orthogonal decomposition of $\mathcal{C}$ then $\mathbf{C}(\mathcal{C})$ deformation retracts onto $\bigoplus_{\gamma \in \Gamma} \mathbf{C}\left(\mathcal{B}_{\gamma}\right)$.

Proof. For the first assertion, recall from Proposition 5.8 that $\mathrm{B}(\mathcal{C})$ deformation retracts onto $\mathbf{B}\left(\mathcal{C}, \mathcal{J}_{\mathcal{B}}\right)$, which induces a deformation retract

$$
\mathbf{C}(\mathcal{C})=\mathbf{B}(\mathcal{C}) /[\mathcal{C}, \mathbf{B}(\mathcal{C})] \stackrel{\sim}{\rightarrow} \mathbf{B}\left(\mathcal{C}, \mathcal{J}_{\mathcal{B}}\right) /\left[\mathcal{C}, \mathbf{B}\left(\mathcal{C}, \mathcal{J}_{\mathcal{B}}\right)\right]=\mathbf{C}(\mathcal{B})
$$

by Remark 5.17 since $\mathcal{B}$ is a full subcategory of $\mathcal{C}$.

To prove the second assertion, we set $\mathcal{B}=\bigcup_{\gamma} \mathcal{B}_{\gamma}$ and recall from Theorem 5.11 that $\mathbf{B}(\mathcal{C})$ deformation retracts onto the semi-orthogonal bar complex $\mathbf{B}^{\Gamma}\left(\mathcal{C}, \mathcal{J}_{\mathcal{B}}\right)$ in the category of $\mathcal{C}, \mathcal{C}$-bimodules. There is an induced deformation retract

$$
\mathbf{C}(\mathcal{C})=\mathbf{B}(\mathcal{C}) /[\mathcal{C}, \mathbf{B}(\mathcal{C})] \stackrel{\simeq}{\rightarrow} \mathbf{B}^{\Gamma}\left(\mathcal{C}, \mathcal{J}_{\mathcal{B}}\right) /\left[\mathcal{C}, \mathbf{B}^{\Gamma}\left(\mathcal{C}, \mathcal{J}_{\mathcal{B}}\right)\right] .
$$

The complex $\mathbf{B}^{\Gamma}\left(\mathcal{C}, \mathcal{J}_{\mathcal{B}}\right)$ is a one-sided twist of

$$
\bigoplus_{r \geq 0} \bigoplus_{X_{1}, \ldots, X_{r+1} \in \mathcal{B}} \Sigma^{r}\left(\mathrm{e} X_{1} \otimes X_{1} \mathrm{e} X_{2} \otimes \cdots \otimes X_{r+1} \mathrm{e}\right)
$$

with $X_{1} \geq X_{2} \geq \cdots \geq X_{r+1}=: X_{0}$. By semi-orthogonality, the summands with $X_{1}>X_{0}$ become contractible in the quotient $\mathbf{B}^{\Gamma}\left(\mathcal{C}, \mathcal{J}_{\mathcal{B}}\right) /\left[\mathcal{C}, \mathbf{B}^{\Gamma}\left(\mathcal{C}, \mathcal{J}_{\mathcal{B}}\right)\right]$. Contracting these induces a deformation retract onto the subcomplex where $X_{1}, \ldots, X_{r}, X_{0} \in \mathcal{B}_{\gamma}$ for some $\gamma$. For each $\gamma$ the contribution of all such terms is the two-sided bar complex $\mathbf{B}\left(\mathcal{B}_{\gamma}\right)$. By inspection the differential preserves summands, and we obtain the direct sum decomposition in the statement.

The following corollary was proved in [Kuz09].

Corollary 5.20. Suppose $\left\{\mathcal{B}_{\gamma} \subset \mathcal{C}\right\}$ defines a $\Gamma$-indexed semi-orthogonal decomposition of $\mathcal{C}$. Then we have

$$
\mathrm{HH}_{\mathbf{0}}(\mathcal{C}) \cong \bigoplus_{\gamma \in \Gamma} \mathrm{HH}_{\mathbf{r}}\left(\mathcal{B}_{\gamma}\right) .
$$

Corollary 5.21. If $\mathcal{C}$ is a dg category, then the cyclic bar complex of $\operatorname{Pretr}(\mathcal{C})$ deformation retracts onto the cyclic bar complex of $\mathcal{C}$, and we have a natural isomorphism $\mathrm{HH} .(\mathrm{C}) \cong$ HH. $(\operatorname{Pretr}(\mathcal{C}))$ induced by the inclusion $\mathrm{e} \hookrightarrow \operatorname{Pretr}(\mathcal{C})$.

Example 5.22. If $\mathcal{A}$ is an additive category (regarded as a dg category trivially) then we have the natural isomorphism HH. $(\mathcal{A}) \cong \mathrm{HH} .\left(\mathrm{Ch}^{b}(\mathcal{A})\right)$ induced by the inclusion $\mathcal{A} \hookrightarrow \mathrm{Ch}^{b}(\mathcal{A})$.

If $X \in \mathcal{C}$ is an object, then we write $[X]:=\left[\operatorname{Id}_{X}\right]$ for its class in $\mathrm{HH}_{0}(\mathcal{C}) \subset \mathrm{HH}$. (e). If $X$ is a one-sided twisted complex constructed from objects $X^{i}$, it is natural to ask for the relation between the classes $[X]$ and $\left[X^{i}\right]$.

Lemma 5.23. For objects $X, Y \in \mathcal{C}$ and $f: X \rightarrow Y$, we have:

$$
[X \oplus Y]=[X]+[Y]
$$




$$
\begin{gathered}
{\left[\Sigma^{1} X\right]=-[X]} \\
{[\operatorname{Cone}(f: X \rightarrow Y)]=[Y]-[X]}
\end{gathered}
$$

Proof. It is sufficient to prove (13c). Let $Z=\operatorname{Cone}(X \stackrel{f}{\rightarrow} Y)$, let $i_{X}, i_{Y}$ denote the inclusions of $X$ and $Y$ into $Z$ and let $\pi_{X}, \pi_{Y}$ denote the projections of $Z$ onto $X$ and $Y$. Note that $i_{X}$ and $\pi_{X}$ have degrees \pm 1 , and

$$
\begin{gathered}
d_{\mathfrak{C}}\left(i_{X}\right)=i_{Y} f, \quad d_{\mathfrak{C}}\left(i_{Y}\right)=0, \quad d_{\mathfrak{e}}\left(\pi_{X}\right)=0, \quad d_{\mathfrak{e}}\left(\pi_{Y}\right)=-f \pi_{X}, \\
\pi_{X} i_{X}=\operatorname{Id}_{X}, \quad \pi_{Y} i_{Y}=\operatorname{Id}_{Y}, \quad i_{X} \pi_{X}+i_{Y} \pi_{Y}=\operatorname{Id}_{Z} .
\end{gathered}
$$

So we compute

$$
\begin{gathered}
d_{\mathbf{C}}\left(\left\|i_{X}\right\| \pi_{X}\right)=-\left\|\operatorname{Id}_{X}-\right\| i_{X} \pi_{X}-\left\|i_{Y} f\right\| \pi_{X}, \\
d_{\mathbf{C}}\left(\left\|i_{Y}\right\| \pi_{Y}\right)=\left\|\operatorname{Id}_{Y}-\right\| i_{Y} \pi_{Y}+\left\|i_{Y}\right\| f \pi_{X}, \\
d_{\mathbf{C}}\left(\left\|i_{Y}\right\| f \| \pi_{X}\right)=0-\left\|i_{Y} f\right\| \pi_{X}+\left\|i_{Y}\right\| f \pi_{X},
\end{gathered}
$$

since $\pi_{X} i_{Y}=0$ (each of the above has cohomological degree -1 before applying $d_{\mathbf{C}}$ ). Therefore

$$
d_{\mathbf{C}}\left(\left\|i_{Y}\right\| f\left\|\pi_{X}-\right\| i_{X}\left\|\pi_{X}-\right\| i_{Y} \| \pi_{Y}\right)=\| \operatorname{Id}_{Z}-\left(\left\|\operatorname{Id}_{Y}-\right\| \operatorname{Id}_{X}\right),
$$

which shows that $\left[\operatorname{Id}_{Z}\right] \simeq\left[\operatorname{Id}_{Y}\right]-\left[\operatorname{Id}_{X}\right]$ in $\operatorname{HH}_{0}(\mathcal{C})$.

Corollary 5.24. Let $\mathcal{C}$ be a dg category and consider an object $X=\operatorname{tw}_{\alpha}\left(\bigoplus_{i} X^{i}\right)$ in $\operatorname{Pretr}(\mathcal{C})$, then under the identification $\mathrm{HH}$. $(\operatorname{Pretr}(\mathrm{C})) \cong \mathrm{HH}$. (e) the class of $X$ is given by the Euler characteristic

$$
[X]=\sum_{i}(-1)^{i}\left[X^{i}\right] .
$$

5.6. Connes differential and HKR isomorphism. The cyclic bar complex of a dg category has a canonical differential $\mathbb{B}$ of cohomological degree -1 [LQ84] that we now describe. Given $\left\|f_{1}\right\| \cdots \| f_{r+1} \in \mathbf{C}^{-r}(\mathcal{C})$ with $f_{1} \in X_{1} \mathcal{C} X_{2}$, we define

$$
\begin{aligned}
& t\left(\left\|f_{1}\right\| \cdots \| f_{r+1}\right)=(-1)^{\left|f_{r+1}\right|\left(\left|f_{1}\right|+\cdots+\left|f_{r}\right|\right)}\left\|f_{r+1}\right\| f_{1}\|\cdots\| f_{r} \\
& s\left(\left\|f_{1}\right\| \cdots \| f_{r+1}\right)=\left\|\operatorname{Id}_{X_{1}}\right\| f_{1}\|\cdots\| f_{r+1} .
\end{aligned}
$$

Now we define $N=1+\ldots+t^{r}$ and $\mathbb{B}=(1-t) s N$. The operator $\mathbb{B}$ is sometimes called Connes differential.

Example 5.25. For $\| f_{1} \in X_{1} \mathcal{C} X_{1} \subset \mathbf{C}^{0}(\mathcal{C})$ we have

$$
\mathbb{B}\left(\| f_{1}\right)=\left\|\operatorname{Id}_{X_{1}}\right\| f_{1}-\left\|f_{1}\right\| \operatorname{Id}_{X_{1}} \in \mathbf{C}^{-1}(\mathcal{C}) .
$$

For $\left\|f_{1}\right\| f_{2} \in \Sigma^{1}\left(X_{1} \mathcal{e} X_{2} \otimes X_{2} \mathcal{C} X_{1}\right) \subset \mathbf{C}^{-1}(\mathcal{C})$ we have

$$
\begin{gathered}
\mathbb{B}\left(\left\|f_{1}\right\| f_{2}\right)=(1-t)\left(\left\|\operatorname{Id}_{X_{1}}\right\| f_{1}\left\|f_{2}+(-1)^{\left|f_{2} \| f_{2}\right|}\right\| \operatorname{Id}_{X_{2}}\left\|f_{2}\right\| f_{1}\right) \\
=\left\|\operatorname{Id}_{X_{1}}\right\| f_{1}\left\|f_{2}+(-1)^{\left|f_{2} \|\right| f_{1} \mid}\right\| \operatorname{Id}_{X_{2}}\left\|f_{2}\right\| f_{1}-(-1)^{\left|f_{2} \|\right| f_{1} \mid}\left\|f_{2}\right\| \operatorname{Id}_{X_{1}}\left\|f_{1}-\right\| f_{1}\left\|\operatorname{Id}_{X_{2}}\right\| f_{2} .
\end{gathered}
$$

The following is well known.

Lemma 5.26 ([LQ84]). We have $d_{\mathbf{C}} \mathbb{B}+\mathbb{B} d_{\mathbf{C}}=0$ and $\mathbb{B}^{2}=0$. 
Theorem 5.27 ([HKR62, LQ84]). Let $A$ be the ring of functions on a smooth affine scheme $X$. Then there is an algebra isomorphism

$$
\Omega^{\bullet}(X) \simeq \text { HH. }(A),
$$

which identifies de Rham differential $D$ on the algebra of differential forms $\Omega^{*}(X)$ on the left with the (induced) Connes differential $\mathbb{B}$ on the right hand side.

Example 5.28. We have $\mathrm{HH}_{1}(A)=A \otimes A /(a b \otimes c-a \otimes b c+a c \otimes b)$. We can identify this with $\Omega^{1}(X)$ by sending $a \otimes b \rightarrow a D(b)$. Indeed, $a D(b c)=a b D(c)+a c D(b)$. Now $\mathbb{B}(a)=1 \otimes a-a \otimes 1 \in A \otimes A$ is identified with $D(a)-a D(1)=D(a) \in \Omega^{1}(X)$.

Example 5.29. Let $R=\mathbb{C}\left[x_{1}, \ldots, x_{n}\right]$ be the algebra of functions on $\mathbb{C}^{n}$. Then

$$
\mathrm{HH}_{k}(R) \simeq \Omega^{k}\left(\mathbb{C}^{n}\right) \simeq \mathbb{C}\left[x_{1}, \ldots, x_{n}\right] \otimes \wedge^{k}\left[\theta_{1}, \ldots, \theta_{n}\right],
$$

where $\theta_{i}=D\left(x_{i}\right)$. The de Rham differential can be written as

$$
D=\sum_{i} \theta_{i} \frac{\partial}{\partial x_{i}}
$$

\section{THE DG MONOIDAL CENTER AND TRACE}

6.1. Monoidal dg categories and the shuffle product. Note that any (dg) algebra $A$ can be regarded as a dg category with one object. By the same token, a commutative $(\mathrm{dg})$ algebra $A$ can be viewed as a monoidal dg category with one object.

A monoidal dg category is a dg category $\mathcal{C}$ equipped with an object $\mathbb{1} \in \mathcal{C}$, a functor $\star: \mathcal{C} \otimes_{\mathrm{k}} \mathrm{C} \rightarrow \mathcal{C}$, and closed degree zero natural isomorphisms (associator and unitors)

$$
(X \star Y) \star Z \cong X \star(Y \star Z), \quad \mathbb{1} \star X \cong X \cong X \star \mathbb{1}
$$

satisfying the usual coherence relations for monoidal categories (on the nose; not up to homotopy). The associator and unitor isomorphisms will usually be suppressed from the notation, and we will refer to $(\mathcal{C}, \star, \mathbb{1})$ as a $\mathrm{dg}$ monoidal category. If the associators and unitors are in fact identity morphisms, then $(\mathcal{C}, \star, \mathbb{1})$ is said to be strictly monoidal. The usual Eckmann-Hilton argument shows that Ende( $\mathbb{1})$ is always a commutative dg algebra.

We say that a dg monoidal category has duals if every object $X$ has a left dual $X^{*}$ and a right dual ${ }^{*} X$ with evaluation and coevaluation maps

$$
\mathrm{ev}_{X}: X^{*} \star X \rightarrow \mathbb{1}, \quad \operatorname{coev}_{X}: \mathbb{1} \rightarrow X \star X^{*}, \quad \mathrm{ev}_{X}^{\prime}: X \star{ }^{*} X \rightarrow \mathbb{1}, \quad \operatorname{coev}_{X}^{\prime}: \mathbb{1} \rightarrow{ }^{*} X \star X
$$

satisfying the usual string-straightening axioms. Note that the existence of duals is a property of $\mathrm{C}$.

For a $\mathrm{dg}$ monoidal category $\mathcal{C}$ with duals, the operations $(-)^{*}$ and ${ }^{*}(-)$ extend to (contravariant) monoidal dg functors. A pivotal structure on $\mathcal{C}$ is a monoidal natural isomorphism between $(-)^{*}$ and $\left.*^{*}-\right)$ (equivalently, a monoidal natural isomorphism between $\operatorname{Id}_{e}$ and $\left.(-)^{* *}\right)$. 
We say a dg monoidal category $\mathcal{C}$ is strictly pivotal if it is strictly monoidal and we have $X^{*}={ }^{*} X$ for every object $X$, with the identity natural transformation as pivotal structure.

A monoidal structure on $\mathrm{C}$ endows the two-sided bar complex $\mathbf{B}(\mathcal{C}, \mathcal{J})$ with the additional structure of an algebra, via the so-called shuffle product (e.g. [LQ84, EZ53]) which we recall below.

Suppose $\mathcal{C}$ is a $\mathrm{dg}$ monoidal category. Let

$$
\underline{f}=f_{0}\|\cdots\| f_{r+1} \in \Sigma^{r}\left(X_{0} \mathcal{C} X_{1} \otimes \cdots \otimes X_{r+1} \mathcal{C} X_{r+2}\right) \subset \mathbf{B}_{r}(\mathcal{C}, \mathcal{J})
$$

and

$$
\underline{g}=g_{0}\|\cdots\| g_{s+1} \in \Sigma^{s}\left(Y_{0} \mathcal{C} Y_{1} \otimes \cdots \otimes Y_{s+1} \mathcal{C} Y_{s+2}\right) \subset \mathbf{B}_{s}(\mathcal{C}, \mathcal{J})
$$

be two elements in $\mathbf{B}(\mathcal{C}, \mathcal{J})$. To define the product $\underline{f} * \underline{g}$ we set

$$
e_{i}= \begin{cases}f_{i} \star \mathrm{Id} & 1 \leq i \leq r \\ \mathrm{Id} \star g_{i-r} & r<i \leq r+s\end{cases}
$$

where we keep an open mind about what objects the identity morphisms are associated with. Now we set

$$
\underline{f} * \underline{g}:=(-1)^{|\underline{f}| s} \sum_{\pi \in S_{(r, s)}}(-1)^{w \sigma(\pi, \underline{f}, \underline{g})}\left(f_{0} \star g_{0}\right)\left\|e_{\pi(1)}\right\| \cdots\left\|e_{\pi(r+s)}\right\|\left(f_{r+1} \star g_{s+1}\right)
$$

where $S_{(r, s)} \subset S_{r+s}$ denotes the set of shuffle permutations, and $w \sigma(\pi, \underline{f}, g)$ denotes the weighted sign of the permutation $\pi$, to which a transposition of $f_{i} \star \overline{\mathrm{I}} \mathrm{d}$ and $\mathrm{Id} \star g_{j}$ contributes $(-1)^{\left|f_{i}\right|\left|g_{j}\right|}$. For the $e_{\pi(i)}$ in the summands of this formula, we implicitly choose the identity morphism factor which makes the sequence of morphisms in the summand composable. Note that different summands require different choices, although this is suppressed in the notation.

The shuffle product together with the coproduct $\Delta$ give the two-sided bar complex $\mathbf{B}(\mathcal{C}, \mathcal{J})$ the structure of a bialgebra.

6.2. The quadmodule associated to a dg monoidal category. Let $\mathcal{C}$ now be a $\mathrm{dg}$ monoidal category. Fix objects $X_{1}, X_{2}, Y_{1}, Y_{2} \in \mathcal{C}$, and let $f \in \operatorname{Hom}_{\mathcal{C}}\left(X_{1} \star X_{2}, Y_{1} \star Y_{2}\right)$ be given. We also consider objects $X_{i}^{\prime}, Y_{i}^{\prime} \in \mathcal{C}(i=1,2)$ and morphisms

$$
a_{i} \in \operatorname{Hom}_{\mathcal{C}}\left(Y_{i}, Y_{i}^{\prime}\right), \quad b_{i} \in \operatorname{Hom}_{\mathcal{C}}\left(X_{i}^{\prime}, X_{i}\right) .
$$

Then we define the following operations:

$$
\begin{array}{ll}
a_{1} \circ_{1} f:=\left(a_{1} \star \operatorname{Id}_{Y_{2}}\right) \circ f, & a_{2} \circ_{2} f:=\left(\operatorname{Id}_{Y_{1}} \star a_{2}\right) \circ f, \\
f \circ_{1} b_{1}:=f \circ\left(b_{1} \star \operatorname{Id}_{X_{2}}\right), & f \circ_{2} b_{2}:=f \circ\left(\operatorname{Id}_{X_{1}} \star b_{2}\right) .
\end{array}
$$

These operations give a $\left(\mathcal{C} \otimes_{\mathbb{k}} \mathcal{C}\right),\left(\mathcal{C} \otimes_{\mathbb{k}} \mathcal{C}\right)$-bimodule structure on

$$
X:=\bigoplus_{X_{1}, X_{2}, Y_{1}, Y_{2}}\left(Y_{1} \star Y_{2}\right) \mathcal{C}\left(X_{1} \star X_{2}\right)
$$


We may regard $X$ just defined as a quadmodule over $\mathcal{C}$. By combining the monoidal structure and composition of morphisms in $\mathcal{C}$, we have morphisms

$$
\mu_{\nwarrow}, \mu_{\nearrow}: X \otimes_{\mathbb{k}} X \rightarrow X
$$

defined by

$$
\mu_{\nwarrow}(f, g):=(f \star \operatorname{Id}) \circ(\operatorname{Id} \star g)
$$

and

$$
\mu_{\nearrow}(f, g):=(\mathrm{Id} \star f) \circ(g \star \mathrm{Id})
$$

whenever these compositions make sense. These operations interact with the quadmodule structure according to

$$
\begin{aligned}
\mu_{\nwarrow}\left(f \circ_{2} a, g\right) & =\mu_{\nwarrow}\left(f, a \circ_{1} g\right) \\
\mu_{\nwarrow}\left(a \circ_{1} f, g \circ_{2} b\right) & =a \circ_{1} \mu_{\nwarrow}(f, g) \circ_{2} b \\
\mu_{\nwarrow}\left(a \circ_{2} f, a^{\prime} \circ_{2} g\right) & =\left(a \star a^{\prime}\right) \circ_{2} \mu_{\nwarrow}(f, g) \\
\mu_{\nwarrow}\left(f \circ_{1} b, g \circ_{1} b^{\prime}\right) & =\mu_{\nwarrow}(f, g) \circ_{1}\left(b \star b^{\prime}\right),
\end{aligned}
$$

with similar identities involving $\mu_{\nearrow}$ (swapping the roles of $\circ_{1}$ and $\circ_{2}$ ).

From $X$ we obtain a $\mathcal{C}, \mathcal{C}$-bimodule by forgetting the "northeast" and "southwest" actions of $\mathcal{C}$. Precisely, $X_{12}$ equals $X$, but with $\mathcal{C}, \mathcal{C}$-bimodule structure

$$
a \otimes f \otimes b \mapsto a \circ_{1} f \circ_{2} b, \quad a, b \in \mathcal{C}, \quad f \in X_{12} .
$$

One may define a bimodule $X_{21}$ in a similar fashion, but we will not need it.

Remark 6.1. The map $\mu_{\nwarrow}$ makes $X_{12}$ into an algebra object in $\mathcal{C}$, $\mathcal{C}$-bimodules, with unit $\mathcal{C} \rightarrow X_{12}$ given by the bimodule map sending $\operatorname{Id}_{X} \in \mathcal{C}$ to the canonical isomorphism $\mathbb{1} \star X \rightarrow X \star \mathbb{1}$, regarded as an element of $X x_{12} X$.

Remark 6.2. The map $\mu_{\nearrow}$ also defines an associative multiplication on $X_{12}$ which interacts with the bimodule structure in a nonstandard way:

$$
\begin{aligned}
\mu_{\nearrow}\left(f \circ_{1} a, g\right) & =\mu_{\nearrow}\left(f, a \circ_{2} g\right) \\
\mu_{\nearrow}\left(a \circ_{2} f, g \circ_{1} b\right) & =a \circ_{2} \mu_{\nearrow}(f, g) \circ_{1} b \\
\mu_{\nearrow}\left(a \circ_{1} f, a^{\prime} \circ_{1} g\right) & =(-1)^{\left|a^{\prime}\right|(|a|+|f|)}\left(a^{\prime} \star a\right) \circ_{1} \mu_{\nearrow}(f, g) \\
\mu_{\nearrow}\left(f \circ_{2} b, g \circ_{2} b^{\prime}\right) & =(-1)^{|b|\left(\left|b^{\prime}\right|+|g|\right)} \mu_{\nearrow}(f, g) \circ_{2}\left(b^{\prime} \star b\right),
\end{aligned}
$$

By fixing $Y_{2}=Z^{\prime}$ and $X_{1}=Z$, we obtain sub-bimodules of $X_{12}$ of the form

$$
x_{12}\left(Z^{\prime}, Z\right):=\bigoplus_{X, Y \in \mathcal{C}}\left(Y \star Z^{\prime}\right) \mathrm{e}(Z \star X) \subset x_{12}
$$

Note that $\mu_{\nwarrow}$ restricts to morphisms of $\mathcal{C}$, $\mathrm{C}$-bimodules

$$
\mu_{\nwarrow}: X_{12}\left(Z^{\prime}, Z\right) \otimes_{\mathrm{e}} X_{12}\left(U^{\prime}, U\right) \rightarrow X_{12}\left(Z^{\prime} \star U^{\prime}, Z \star U\right),
$$

while $\mu \nearrow$ restricts to morphisms

$$
\mu_{\nearrow}: X_{12}\left(Z^{\prime \prime}, Z^{\prime}\right) \otimes_{\mathbb{k}} X_{12}\left(Z^{\prime}, Z\right) \rightarrow X_{12}\left(Z^{\prime \prime}, Z\right)
$$


In particular the bimodule $X_{12}(Z):=X_{12}(Z, Z)$ inherits an associative multiplication (which respects the $\mathrm{dg} \mathbb{k}$-module structure and is compatible with the $\mathcal{C}, \mathcal{C}$-bimodule structure).

6.3. The dg monoidal centralizer. Let $\mathcal{C}$ be a dg monoidal category, and fix an object $Z \in \mathcal{C}$. We would like to discuss what it means for $Z$ to be central in $\mathcal{C}$. Just as in the usual Drinfeld center, this is not a property enjoyed by $Z$, but rather additional structure which must be provided.

Actually, we will consider the slightly broader problem of defining what it means for $Z$ to centralize a full $\mathrm{dg}$ monoidal subcategory $\mathcal{M} \subset \mathcal{C}$. For $Z$ to centralize $\mathcal{M}$ (up to homotopy) requires the following data:

(1) for each object $X \in \mathcal{M}$, a degree zero closed morphism, called half-braiding, $\tau_{X} \in(X \star Z) \mathcal{e}(Z \star X)$.

(2) for each closed morphism $f \in Y \mathcal{M} X$ a homotopy $h_{f} \in(X \star Z) \mathcal{C}(Z \star X)$ with $d_{\mathfrak{e}}\left(h_{f}\right)=f \circ_{1} \circ \tau_{X}-\tau_{Y} \circ_{2} f$

(3) certain higher homotopies.

To get a feeling for the sort of higher homotopies required, observe that for each pair of closed morphisms $f_{0} \in X_{0} \mathcal{M} X_{1}$ and $f_{1} \in X_{1} \mathcal{M} X_{2}$ we have two ways of commuting $f_{0} \circ f_{1}$ past $Z$. First, we have the homotopy $h_{f_{0} \circ f_{1}}$. But we also have $h_{f_{0}} \circ_{2} f_{1}+$ $(-1)^{\left|f_{0}\right|} f_{0} \circ \circ_{1} \circ h_{f_{1}}$. We should require the difference of these two homotopies (which is a closed morphism of degree -1 ) to be null-homotopic. The various higher homotopies required are, in fact, already organized for us in the form of the two-sided bar complex.

Definition 6.3. Let $\mathcal{C}$ be a $d g$ monoidal category and $\mathcal{M} \subset \mathcal{C}$ a subcategory. The $d g$ monoidal centralizer of $\mathcal{M}$ in $\mathcal{C}$ is the dg category $\mathcal{Z}_{\mathrm{e}}^{\mathrm{dg}}(\mathcal{M})$ whose objects are pairs $(Z, \tau)$ where $Z \in \mathcal{C}$ and $\tau: \mathbf{B}(\mathcal{M}) \rightarrow X_{12}(Z)$ is a map of $\mathcal{C}$, $\mathcal{C}$-bimodules as well as a map of dg algebras. The complex of morphisms in $\mathcal{Z}_{\mathrm{e}}^{\mathrm{dg}}(\mathcal{M})$ from $(Z, \tau)$ to $\left(Z^{\prime}, \tau^{\prime}\right)$ is the subcomplex of $\operatorname{Hom}_{\mathfrak{e}}\left(Z, Z^{\prime}\right)$ consisting of those morphisms which commute strictly with the structure maps in the images of $\mathbf{B}(\mathcal{M})$. I.e. those $z \in \operatorname{Hom}_{\mathcal{C}}\left(Z, Z^{\prime}\right)$ such that for $f \in Y \mathbf{B}(\mathcal{M}) X$ we have

$$
z \circ_{2} \tau(f)=\tau^{\prime}(f) \circ_{1} z
$$

The $d g$ Drinfeld center is defined to be $\mathcal{Z}^{\mathrm{dg}}(\mathcal{C}):=\mathcal{Z}_{\mathfrak{C}}^{\mathrm{dg}}(\mathcal{C})$.

Remark 6.4. It is perhaps better to weaken the condition on morphisms in $\mathcal{Z}^{\mathrm{dg}}(\mathcal{C})$ to strictly commute with the structure maps from $\mathbf{B}(\mathcal{C})$. This would bring us into the world of $A_{\infty}$-categories, which we choose to avoid for the moment. In the rest of this paper, we only consider objects of the dg Drinfeld center and not morphisms.

The dg Drinfeld center $\mathcal{Z}^{\mathrm{dg}}(\mathcal{C})$ has a tensor product defined by

$$
(Z, \tau) \star\left(Z^{\prime}, \tau^{\prime}\right):=\left(Z \star Z^{\prime}, \tau^{\prime \prime}\right),
$$

where $\tau^{\prime \prime}$ is the composition of maps

$$
\mathbf{B}(\mathcal{e}) \rightarrow \mathbf{B}(\mathcal{e}) \otimes_{\mathfrak{e}} \mathbf{B}(\mathfrak{e}) \rightarrow X_{12}(Z) \otimes_{\mathfrak{e}} X_{12}\left(Z^{\prime}\right) \rightarrow X_{12}\left(Z \star Z^{\prime}\right)
$$


Here, the first map is the coproduct on the two-sided bar complex, and the second map sends

$$
f \otimes f^{\prime} \mapsto\left(f \star \operatorname{Id}_{Z^{\prime}}\right) \star\left(\operatorname{Id}_{Z} \star f^{\prime}\right) \in X^{\prime \prime} X_{12}\left(Z \star Z^{\prime}\right) X
$$

for all $f^{\prime} \in X^{\prime} X_{12}\left(Z^{\prime}\right) X$ and all $f \in X^{\prime \prime} X_{12}(Z) X^{\prime}$.

The following is immediate.

Proposition 6.5. There is a natural forgetful functor $\mathcal{Z}^{\text {dg }}(\mathcal{C}) \rightarrow \mathcal{C}$. It is monoidal.

Remark 6.6. It is well known that the Drinfeld center of a monoidal category is braided. It is natural to expect that dg Drinfeld center is braided in a dg sense, that is, the braiding is natural up to homotopy. To define such a structure, it seems likely that passage to the world of $A_{\infty}$ (braided monoidal) categories and functors is unavoidable. We save such explorations for future work.

Remark 6.7. If $\mathcal{C}$ is a monoidal category, considered as a dg category with trivial differential, then the Drinfeld center $\mathcal{Z}^{0}(\mathcal{C})$ is a monoidal subcategory of $\mathcal{Z}^{\mathrm{dg}}(\mathcal{C})$.

In the following remarks we spell out the meaning of some of the structure maps that are part of the data of a central object $(Z, \tau)$.

Remark 6.8. For each $X \in \mathcal{C}$ the two-sided bar complex has a distinguished degree zero closed element $\operatorname{Id}_{X} \| \operatorname{Id}_{X}$. The image of this element in $X_{12}(Z)$ under $\tau$ will be denoted $\tau_{X}$ and is called the half-braiding of $X$ with $Z$. Then each degree zero element $a \| b$ gets sent to $a \circ_{1} \tau_{X} \circ_{2} b$ where $X$ is the codomain of $b$ (same as the domain of $a$ ), since $\tau$ commutes with the bimodule structures on $\mathbf{B}(\mathcal{C})$ and $X_{12}(Z)$.

Remark 6.9. In $\mathbf{B}(\mathcal{C})$ we have $\left(\operatorname{Id}_{X} \| \operatorname{Id}_{X}\right) *\left(\operatorname{Id}_{Y} \| \operatorname{Id}_{Y}\right)=\operatorname{Id}_{X \star Y} \| \operatorname{Id}_{X \star Y}$. By definition of the center, $\tau$ is a map of dg algebras, which implies

$$
\tau_{X \star Y}=\left(\operatorname{Id}_{X} \star \tau_{Y}\right) \circ\left(\tau_{X} \star \operatorname{Id}_{Y}\right)
$$

and one recovers the familiar compatibility between half-braiding morphisms and the monoidal structure in $\mathrm{C}$.

Remark 6.10. For each morphism $f \in Y \complement X$ of degree $l$ the two-sided bar complex has a degree $l-1$ element of the form $\operatorname{Id}_{Y}\|f\| \operatorname{Id}_{X}$, whose image under $\tau$ we denote by $h_{f}$. If $f$ is closed then we have

$$
d\left(h_{f}\right)=d\left(\tau\left(\operatorname{Id}_{Y}\|f\| \operatorname{Id}_{X}\right)\right)=\tau\left(f\left\|\operatorname{Id}_{X}-\operatorname{Id}_{Y}\right\| f\right)=f \circ_{1} \tau_{X}-\tau_{Y} \circ_{2} f,
$$

since $\tau$ commutes with the differentials in $\mathbf{B}(\mathcal{C})$ and $X_{12}(Z)$. This means $h_{f}$ is a homotopy for commuting $f$ through the half-braiding with $Z$.

Similarly for each sequence of composable (closed) morphisms $f, g$ we have an element $h_{f, g}=\tau(\|f\| g \|)$ (dropping explicit occurrences of identity maps) satisfying

$$
\begin{aligned}
d\left(h_{f, g}\right) & =d(\tau(\|f\| g \|)) \\
& =\tau(d(\|f\| g \|)) \\
& =\tau(f\|g\|-\|f \circ g\|+\|f\| g) \\
& =(-1)^{|f|} f \circ_{1} h_{g}-h_{f \circ g}+h_{f} \circ_{2} g
\end{aligned}
$$


and so on. In summary, we see that $\tau$ gives the data of a half-braiding with $Z$ that is natural up to coherent homotopy.

Remark 6.11. If $\mathcal{C}$ is an arbitrary dg category, there is a functor from $Z^{d g}(\mathcal{C})$ to the usual Drinfeld center of the homotopy category $Z^{0}\left(H^{0}(\mathcal{C})\right)$. It sends a central object $(Z, \tau)$ to $\left(Z, \tau_{X}\right)$ and forgets all higher homotopies.

Lemma 6.12. If $(Z, \tau)$ is an object of $\mathcal{Z}^{\text {dg }}(\mathcal{C})$ and $X$ an object of $\mathcal{C}$ that has a right dual, then $\tau_{X}: X \star Z \rightarrow X \star Z$ is a homotopy equivalence.

Proof. If ${ }^{*} X$ is a right dual of $X$ with

$$
\mathrm{ev}^{\prime}: X \star^{*} X \rightarrow \mathbb{1}, \quad \operatorname{coev}^{\prime}: \mathbb{1} \rightarrow{ }^{*} X \star X
$$

then we set

$$
\tau_{X}^{-1}:=\left(\mathrm{ev}^{\prime} \star \operatorname{Id}_{Z \star X}\right) \circ\left(\operatorname{Id}_{X} \star \tau_{* X} \star \operatorname{Id}_{* X}\right) \circ\left(\operatorname{Id}_{X \star Z} \star \operatorname{coev}^{\prime}\right)
$$

and one can check that $\tau_{X} \tau_{X}^{-1} \simeq \operatorname{Id}_{X \star Z}$ and $\tau_{X}^{-1} \tau_{X} \simeq \operatorname{Id}_{Z \star X}$.

Remark 6.13. The $\mathrm{dg}$ monoidal center $\mathcal{Z}^{\mathrm{dg}}(\mathcal{C})$ embeds as a monoidal $\mathrm{dg}$ subcategory of $\mathcal{Z}^{\mathrm{dg}}(\operatorname{Pretr}(\mathcal{C}))$. To see this, let $(Z, \tau)$ be an object of $\mathcal{Z}^{\mathrm{dg}}(\mathcal{C})$. The half-braiding morphism $\tau$ extends to $\Sigma \mathcal{C}$ in a trivial fashion, so $(Z, \tau)$ can be thought of as an object of $\mathcal{Z}^{\operatorname{dg}}(\Sigma \mathcal{C})$. Next, given a morphism $f: X \rightarrow Y$, the half-braiding of the cone Cone $(f):=[X \stackrel{f}{\rightarrow} Y]$ past $Z$ is defined by the following morphism:

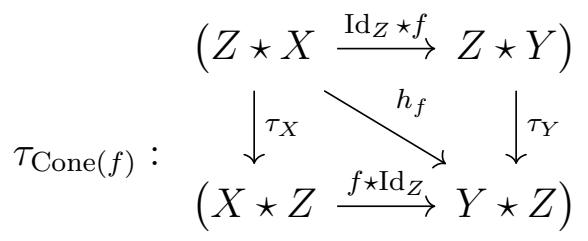

Furthermore, the (higher) homotopies for half-braidings for cones are analogously determined by $\tau$. Iterating this construction, we see that $(Z, \tau)$ is derived central for one-sided twisted complexes, i.e. it represents an object in $\mathcal{Z}^{\operatorname{dg}}(\operatorname{Pretr}(\mathcal{C}))$.

Remark 6.14. Given an object $(Z, \tau)$ in $\mathcal{Z}^{\mathrm{dg}}(\mathcal{C})$, it is instructive to compute the halfbraiding of twisted complexes past $Z$ explicitly. Thus, suppose we have a twisted complex $\operatorname{tw}_{\alpha}(X)$ with $X \in \mathcal{C}$ (or $\left.X \in \Sigma \mathcal{C}\right)$.

Note that $Z \star \operatorname{tw}_{\alpha}(X)=\operatorname{tw}_{\operatorname{Id}_{Z} \star \alpha}(Z \star X)$ and $\operatorname{tw}_{\alpha}(X) \star Z=\operatorname{tw}_{\alpha \star \operatorname{Id}_{Z}}(X \star Z)$. The half-braiding morphism $Z \star \operatorname{tw}_{\alpha}(X) \rightarrow \operatorname{tw}_{\alpha}(X) \star Z$ can then be constructed using standard homological perturbation theory as:

$$
\begin{aligned}
\tau_{\mathrm{tw}_{\alpha}(X)} & : Z \otimes \operatorname{tw}_{\alpha}(X) \rightarrow \operatorname{tw}_{\alpha}(X) \otimes Z, \\
\tau_{\mathrm{tw}_{\alpha}(X)} & :=\sum_{r \geq 0}(-1)^{\left(\begin{array}{c}
r+1 \\
2
\end{array}\right)} \tau\left(\operatorname{Id}_{X}\|\underbrace{\alpha\|\ldots\| \alpha}_{r}\| \operatorname{Id}_{X}\right) .
\end{aligned}
$$


(This is well-defined if $X$ has the structure of a one-sided twisted complex.) To see that this is a closed degree zero morphism in $\operatorname{Tw}(\Sigma \mathcal{C})$, we must show that

$$
d\left(\tau_{(X, \alpha)}\right)+\left(\alpha \otimes \operatorname{Id}_{Z}\right) \circ \tau_{(X, \alpha)}-\tau_{\mathrm{tw}_{\alpha}(X)} \circ\left(\operatorname{Id}_{Z} \otimes \alpha\right)=0 .
$$

We compute:

$$
\begin{aligned}
& \left.d\left(\tau_{\mathrm{tw}_{\alpha}(X)}\right)=\sum_{r \geq 0}(-1)^{\left({ }^{r+1}\right.}{ }_{2}\right) d\left(\tau\left(\operatorname{Id}_{X}\|\underbrace{\alpha\|\ldots\| \alpha}_{r}\| \operatorname{Id}_{X}\right)\right)
\end{aligned}
$$

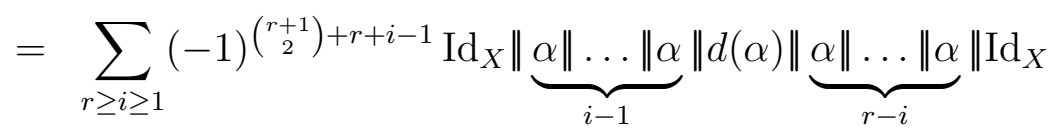

$$
\begin{aligned}
& +\sum_{r \geq 1}(-1)^{\left(\begin{array}{c}
(+1 \\
2
\end{array}\right)+r-1} \alpha \circ \tau\left(\operatorname{Id}_{X}\|\underbrace{\alpha\|\ldots\| \alpha}_{r-1}\| \operatorname{Id}_{X}\right) \\
& +\sum_{r>i \geq 1}(-1)^{\left({ }^{(+1}+1\right)+i} \operatorname{Id}_{X}\|\underbrace{\alpha\|\ldots\| \alpha}_{i-1}\| \alpha^{2}\|\underbrace{\alpha\|\ldots\| \alpha}_{r-i-1}\| \operatorname{Id}_{X} \\
& +\sum_{r \geq 1}(-1)^{\left(\begin{array}{c}
r+1 \\
2
\end{array}\right)+r} \tau\left(\operatorname{Id}_{X}\|\underbrace{\alpha\|\ldots\| \alpha}_{r-1}\| \operatorname{Id}_{X}\right) \circ \alpha \\
& =-\alpha \circ \tau_{\mathrm{tw}_{\alpha}(X)}+(-1)^{\left|\tau_{\mathrm{tw}_{\alpha}(X)}\right|} \tau_{\mathrm{tw}_{\alpha}(X)} \circ \alpha .
\end{aligned}
$$

Since $\left(\begin{array}{c}r+1 \\ 2\end{array}\right)+r-1 \equiv\left(\begin{array}{c}r+2 \\ 2\end{array}\right) \bmod 2$ and $d(\alpha)+\alpha^{2}=0$ the terms in the first and third line cancel and we get $d\left(\tau_{(X, \alpha)}\right)=-\left(\alpha \otimes \operatorname{Id}_{Z}\right) \circ \tau_{(X, \alpha)}+\tau_{(X, \alpha)} \circ\left(\alpha \otimes \operatorname{Id}_{Z}\right)$ as desired.

Remark 6.15. It an exercise to check that the braiding morphisms which commute $Z$ past one-sided twisted complexes satisfy the required compatibility with the monoidal structure.

6.4. The dg monoidal trace. Let $\mathcal{C}$ be a dg monoidal category (which we will soon assume to be strictly monoidal). First, recall the $\mathcal{C}$, $\mathcal{C}$-bimodule $X_{12}$ with

$$
x_{12}:=\bigoplus_{X, Y, Z, Z^{\prime} \in \mathcal{e}} \operatorname{Hom}\left(X \star Z, Z^{\prime} \star Y\right) \quad\left(=\left(Z^{\prime} \star Y\right) \mathfrak{e}(X \star Z)\right),
$$

with bimodule structure defined by

$$
a \cdot f \cdot b:=a \circ_{1} f \circ_{2} b \quad \forall a, b \in \mathcal{C}, \quad f \in X_{12} .
$$

Definition 6.16. We define a dg category $\operatorname{Tr}(\mathcal{C})$, the (dg horizontal) trace of $\mathcal{C}$, as follows. First, define the $\mathcal{C}, \mathcal{C}$ bimodule

$$
\mathbf{B}(\mathcal{e}) \otimes_{e} X_{12}
$$

Elements of this bimodule are linear combinations of symbols of the form $c_{0}\left\|c_{1}\right\| \cdots\left\|c_{r}\right\|\left(c_{r+1} \circ_{1}\right.$ $f$ ) where $c_{0}, \ldots, c_{r+1} \in \mathcal{C}$ is a sequence of composable morphisms and $f \in X_{12}$. Then we identify the left and right $\mathcal{C}$-actions by forming the quotient

$$
\mathbf{C}\left(\mathcal{e}, X_{12}\right):=\left(\mathbf{B}(\mathcal{C}) \otimes_{\mathfrak{e}} X_{12}\right) / \sim
$$


with respect to the relations of the form

$$
(-1)^{s} c_{0}\left\|c_{1}\right\| \cdots\left\|c_{r}\right\| f \simeq \operatorname{Id}\left\|c_{1}\right\| \cdots\left\|c_{r}\right\|\left(f \circ_{2} c_{0}\right)
$$

where the sign is determined by $s=\left|c_{0}\right|\left(\left|c_{1}\right|+\cdots+\left|c_{r}\right|+|f|\right)+\left|c_{0}\right| r$. As usual we will typically drop leftmost identity map from the notation, writing

$$
\left\|c_{1}\right\| \cdots\left\|c_{r}\right\| f=\operatorname{Id}\left\|c_{1}\right\| \cdots\left\|c_{r}\right\| f
$$

Note that $\mathrm{C}\left(\mathcal{C}, X_{12}\right)$ is the Hochschild chain complex of $\mathcal{C}$ with coefficients in the bimodule $X_{12}$. Recall that each pair of objects $X, X^{\prime} \in \mathcal{C}$ determines a subbimodule

$$
x_{12}\left(X^{\prime}, X\right):=\bigoplus_{Y, Y^{\prime}} \operatorname{Hom}_{\mathcal{C}}\left(X \star Y, Y^{\prime} \star X^{\prime}\right) \subset X_{12}
$$

and so we have subcomplexes

$$
\mathbf{C}\left(\mathcal{C}, X_{12}\left(X^{\prime}, X\right)\right) \subset \mathbf{C}\left(\mathcal{C}, X_{12}\right)
$$

Now, define a dg category $\operatorname{Tr}(\mathcal{C})$ as follows. Objects of $\operatorname{Tr}(\mathcal{C})$ are the same as objects of $\mathcal{C}$, though to avoid confusion we will write $\operatorname{Tr}(X)$ for $X \in \mathcal{C}$ regarded as an object of $\operatorname{Tr}(\mathcal{C})$. The complex of morphisms is given by

$$
\mathrm{Hom}_{\operatorname{Tr}(\mathcal{e})}\left(\operatorname{Tr}(X), \operatorname{Tr}\left(X^{\prime}\right)\right):=\mathbf{C}\left(\mathcal{C}, X_{12}\left(X^{\prime}, X\right)\right),
$$

with composition induced induced by the shuffle product on the two-sided bar complex and composition in $\mathrm{C}$.

Explicitly, this means that $\operatorname{Hom}_{\operatorname{Tr}(\mathcal{e})}\left(\operatorname{Tr}(X), \operatorname{Tr}\left(X^{\prime}\right)\right)$ has basis given by formal symbols of the form

$$
(\underline{c}, f):=\left\|c_{1}\right\| \cdots\left\|c_{r}\right\| f
$$

where $r \geq 0, c_{i} \in Y_{i} \mathrm{e} Y_{i+1}$ and $f \in\left(Y_{r+1} \star X^{\prime}\right) \mathcal{C}\left(X \star Y_{1}\right)$ for objects $Y_{1}, \ldots, Y_{r+1} \in \mathcal{C}$, and we abbreviate by writing $\underline{c}=\left(c_{1}, \ldots, c_{r}\right)$. When $r=0$, the sequence $\underline{c}$ is empty, and we will use the notation $(\emptyset, f)$ and $\| f$ interchangeably.

We also allow formal symbols of the form $c_{0}\left\|c_{1}\right\| \cdots\left\|c_{r}\right\| f$ but modulo the relations imposed on Hochschild chains, such an expression equals $\pm\left\|c_{1}\right\| \cdots\left\|c_{r}\right\|\left(f \circ_{2} c_{0}\right)$. We picture these symbols as follows.
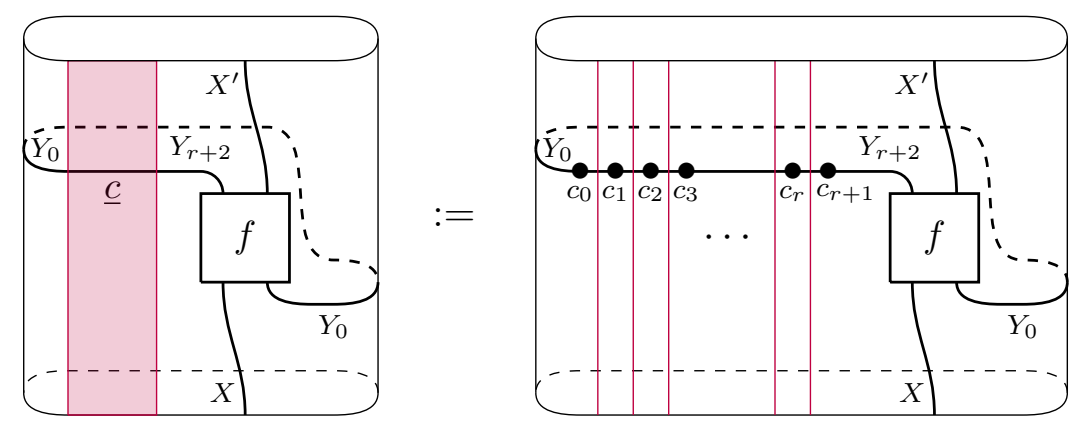

The cohomological grading is

$$
\operatorname{deg}(\underline{c}, f)=-r+|f|
$$

We say that $(\underline{c}, f)$ has bar degree $r$. 
The differential is the usual bar differential (an alternating sum of ways of deleting bars) plus the terms involving the differentials of the individual components:

$$
\begin{aligned}
d_{\operatorname{Tr}(\mathcal{C})}\left(\left\|c_{1}\right\| \cdots\left\|c_{r}\right\| f\right) & =c_{1}\left\|c_{2}\right\| \cdots\left\|c_{r}\right\| f \\
& +\sum_{i=1}^{r-1}(-1)^{i}\left\|c_{1}\right\| \cdots\left\|c_{i} \circ c_{i+1}\right\| \cdots\left\|c_{r}\right\| f \\
& +(-1)^{r}\left\|c_{1}\right\| \cdots\left\|c_{r-1}\right\|\left(c_{r} \circ_{1} f\right) \\
& +\sum_{i=1}^{r}(-1)^{s(i)}\left\|c_{1}\right\| \cdots\left\|d\left(c_{i}\right)\right\| \cdots\left\|c_{r}\right\| f \\
& +(-1)^{s(r+1)}\left\|c_{1}\right\| \cdots\left\|c_{r}\right\| d(f) .
\end{aligned}
$$

where $s(i)=\left|c_{0}\right|+\cdots+\left|c_{i-1}\right|$. When $r=0$ the above generates to $d(\| f)=\| d(f)$.

The composition of morphisms is defined by

$$
(\underline{c}, f) \circ(\underline{d}, g):=\left(\underline{d} * \underline{c}, \mu_{\nearrow}(f, g)\right)
$$

where $*$ denotes the shuffle product from Section 6.1 , and $\mu_{\nearrow}(f, g)$ is as defined in Section 6.2. The composition can be pictured as follows.
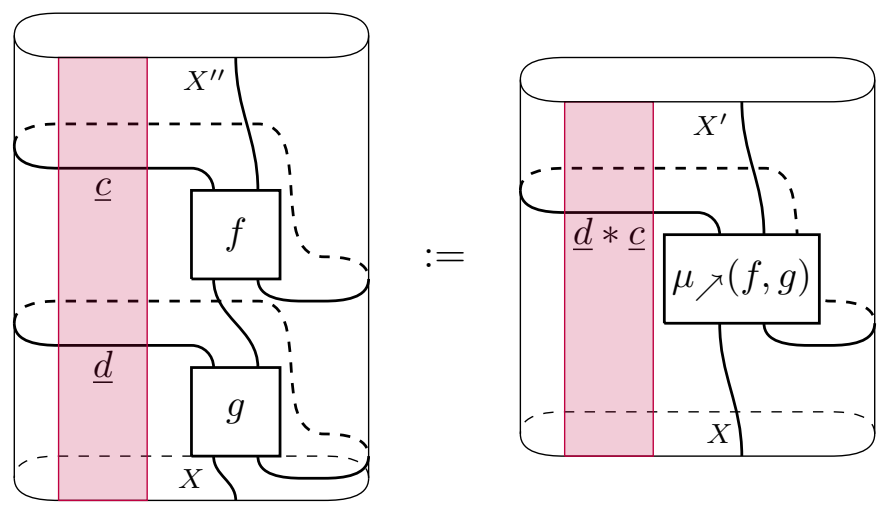

Remark 6.17. The identity endomorphism of $\operatorname{Tr}(X)$ in $\operatorname{Tr}(\mathcal{C})$ is given by $(\emptyset, \phi)$ where $\phi$ is the canonical isomorphism $X \star \mathbb{1} \rightarrow \mathbb{1} \star X$. Then $(\emptyset, \phi) \circ(\emptyset, \phi)$ in $\operatorname{Tr}(\mathcal{C})$ is by definition $(\emptyset, \psi)$ where $\psi$ is the canonical isomorphism $X \star(\mathbb{1} \star \mathbb{1}) \rightarrow(\mathbb{1} \star \mathbb{1}) \star X$. This $\psi$ is homotopic, but not equal to $\phi$. Thus, strictly speaking $(\emptyset, \phi)$ only acts as the identity of $\operatorname{Tr}(X)$ up to homotopy! There is a similar problem concerning the associativity of composition in $\operatorname{Tr}(\mathcal{C})$. The essential issue is that the two-sided bar complex of $\mathcal{C}$ is too large. These annoyances do not arise when $\mathcal{C}$ is strict monoidal, and so this will be assumed en force in the sequel. Without this assumption, $\operatorname{Tr}(\mathcal{C})$ should be regarded as an $A_{\infty}$-category, not a dg category.

We make some observations about $\operatorname{Tr}(\mathcal{C})$ below. First, note that there is a dg functor $\operatorname{Tr}: \mathcal{C} \rightarrow \operatorname{Tr}(\mathcal{C})$ sending $X \mapsto \operatorname{Tr}(X)$ and $f \mapsto \| f$.

Remark 6.18. If $\mathcal{A}$ is a an ordinary monoidal category (regarded as a dg category with trivial grading and differential) then $H^{0}(\operatorname{Tr}(\mathcal{A}))$ is isomorphic to the usual horizontal 
trace $\operatorname{Tr}_{0}(\mathcal{A})$, as defined in [BHLv17, Section 2.4]. In this sense, $\operatorname{Tr}(\mathcal{A})$ is a derived version of $\operatorname{Tr}_{0}(\mathcal{A})$, much as Hochschild homology of an algebra $A$ is a derived version of its trace $A /[A, A]$.

Lemma 6.19. If $\mathcal{A}$ is a $\mathbb{k}$-linear monoidal category, considered as a dg category with trivial grading and differential, then there is a functor $\operatorname{Tr}(\mathcal{A}) \rightarrow \operatorname{Tr}_{0}(\mathcal{A})$ defined on objects by $\operatorname{Tr}(X) \mapsto \operatorname{Tr}_{0}(X)$ and on morphisms by $\| f \mapsto f$ and $(\underline{c}, f) \mapsto 0$ for all sequences of $\underline{c}$ of length $r \geq 1$.

The well-known relationship between vertical and horizontal traces (turn head by 90 degrees) transfers to the derived setting as follows. The following is clear from the definitions.

Proposition 6.20. If $\mathcal{C}$ is a dg monoidal category then

$$
\mathbf{C}_{*}(\mathcal{C})=\operatorname{End}_{\operatorname{Tr}(\mathcal{C})}(\operatorname{Tr}(\mathbb{1})) .
$$

as dg algebras.

Strictly speaking, $\operatorname{End}_{\operatorname{Tr}(\mathcal{C})}(\operatorname{Tr}(\mathbb{1}))$ is given by Hochschild chains of $\mathcal{C}$ with coefficients in the bimodule

$$
\bigoplus_{X, Y \in \mathcal{C}} \operatorname{Hom}(\mathbb{1} \star X, Y \star \mathbb{1})
$$

while $\mathrm{C}_{*}(\mathcal{C})$ is given by Hochschild cochains of $\mathcal{C}$ with coefficients in $\mathcal{C}$. If $\mathcal{C}$ is strict monoidal, then these two bimodules are equal; otherwise they are isomorphic via the unitor maps.

The following says that the natural functor $\mathcal{C} \rightarrow \operatorname{Tr}(\mathcal{C})$ is satisfies a categorical "trace-like" property, provided that $\mathcal{C}$ has duals.

Lemma 6.21. Given two objects $X, Y$ in $\mathrm{C}$, we define the traciator

$$
w_{X, Y}: \operatorname{Tr}(X \star Y) \rightarrow \operatorname{Tr}(Y \star X)
$$

to be the degree zero closed morphism associated to the identity map (or associator in the non-strict monoidal case) $(X \star Y) \star X \rightarrow X \star(Y \star X)$. If $X$ has a right dual in $\mathrm{C}$, then this map is a homotopy equivalence.

Proof. Suppose ${ }^{*} X$ is the right dual to $X$, with structure maps $\operatorname{coev}_{X}: \mathbb{1} \rightarrow{ }^{*} X \star X$ and $\mathrm{ev}_{X}: X \star^{*} X \rightarrow \mathbb{1}$. Define

$$
w_{X, Y}^{-1}: \operatorname{Tr}(Y \star X) \rightarrow \operatorname{Tr}(X \star Y)
$$

to be the composite of

$$
\|\left(\operatorname{coev}_{X} \star \operatorname{Id}_{Y} \star \operatorname{Id}_{X}\right): \operatorname{Tr}(Y \star X) \rightarrow \operatorname{Tr}\left({ }^{*} X \star X \star Y \star X\right),
$$

followed by

$$
w_{* X, X \star Y \star X}: \operatorname{Tr}\left({ }^{*} X \star X \star Y \star X\right) \rightarrow \operatorname{Tr}\left(X \star Y \star X \star{ }^{*} X\right)
$$

and finally

$$
\|\left(\operatorname{Id}_{X} \star \operatorname{Id}_{Y} \star \mathrm{ev}_{X}\right): \operatorname{Tr}\left(X \star Y \star X \star{ }^{*} X\right) \rightarrow \operatorname{Tr}(X \star Y) .
$$

One can now check that $\operatorname{Id}_{X \star Y}-w_{X, Y}^{-1} w_{X, Y}$ and $\operatorname{Id}_{Y \star X}-w_{X, Y} w_{X, Y}^{-1}$ are exact. 
These morphisms can be pictures as follows.

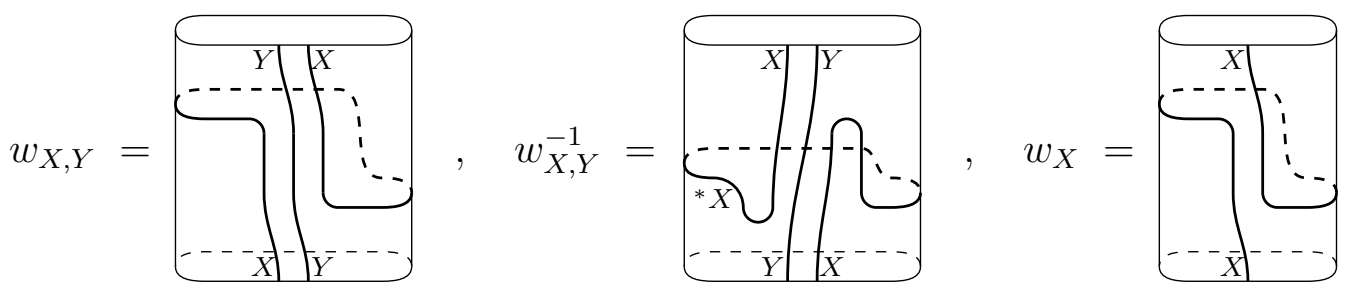

Remark 6.22. It is easy to see that the traciator $w_{X, Y}$ is natural in $Y$, but it is natural in $X$ only up to coherent homotopy. For any closed map $f: X \rightarrow X^{\prime}$ the composition $w_{X^{\prime}, Y} \circ\left(f \star \operatorname{Id}_{Y}\right): \operatorname{Tr}(X \star Y) \rightarrow \operatorname{Tr}\left(Y \star X^{\prime}\right)$ is represented by the map $X \star Y \star X^{\prime} \stackrel{f \star \mathrm{Id} \star \operatorname{Id}}{\longrightarrow}$ $X^{\prime} \star Y \star X^{\prime}$, while the composition $\left(\operatorname{Id}_{Y} \star f\right) \circ w_{X, Y}$ is represented by the map $X \star Y \star$ $X \stackrel{f \star I d \star I d}{\longrightarrow} X \star Y \star X^{\prime}$. The difference of these two maps is given by the boundary of the morphism

$$
w(f ; Y):=\|f\| \operatorname{Id}_{X \star Y \star X^{\prime}}
$$

More generally, for any sequence of composable morphisms $f_{i} \in X_{i} \mathrm{e} X_{i+1}$ for $1 \leq i \leq$ $r$ the derived trace contains higher traciators

$$
w\left(f_{1}\|\ldots\| f_{r} ; Y\right):=\left\|f_{1}\right\| \cdots\left\|f_{r}\right\| \operatorname{Id}_{X_{r+1} \star Y \star X_{1}} \in \operatorname{Hom}_{\operatorname{Tr}}\left(\operatorname{Tr}\left(X_{r+1} \star Y\right), \operatorname{Tr}\left(Y \star X_{1}\right)\right)
$$

which provide the (higher) naturality data for the traciator.

Remark 6.23. For an object $X$ in $\mathcal{C}$ we define the rotator $w_{X}: \operatorname{Tr}(X) \rightarrow \operatorname{Tr}(X)$ to be the endomorphism given by $\| \operatorname{Id}_{X \star X}$. Note that this agrees with the traciator $w_{X, \mathbb{1}}$ as defined in the proof of Lemma 6.21 if $\mathcal{C}$ is strict monoidal. If $X$ has a right dual then $w_{X}$ is invertible up to homotopy.

In this way, the assignment $X \mapsto w_{X}$ is a degree zero endomorphism of the canonical $\mathrm{dg}$ functor $\mathcal{C} \rightarrow \operatorname{Tr}(\mathcal{C})$, natural up to coherent homotopy in the sense of Remark 6.22 .

Remark 6.24. Similar to Remark 6.13, the higher traciators (from (16)) for $\operatorname{Tr}(\mathcal{C})$ already carry enough information to determine traciators in $\operatorname{Tr}(\operatorname{Pretr}(\mathcal{C}))$. For example, given a morphism $f: X \rightarrow X^{\prime}$ and its cone Cone $(f):=\left[X \rightarrow X^{\prime}\right]$, the traciator $w_{\operatorname{Cone}(f), Y}: \operatorname{Tr}(\operatorname{Cone}(f) \star Y) \rightarrow \operatorname{Tr}(Y \star \operatorname{Cone}(f))$ is represented by the morphism

$$
w_{\text {Cone }(f), Y}=\begin{gathered}
\left(\operatorname{Tr}(X \star Y) \stackrel{\operatorname{Tr}\left(f \star I \mathrm{I}_{Y}\right)}{\longrightarrow} \operatorname{Tr}\left(X^{\prime} \star Y\right)\right) \\
\downarrow_{w, Y} \underset{w(f ; Y)}{ } \downarrow w_{X^{\prime}, Y} \\
\left(\operatorname{Tr}(Y \star X) \stackrel{\operatorname{Tr}\left(\mathrm{Id}_{Y} \star f\right)}{\longrightarrow} \operatorname{Tr}\left(Y \star X^{\prime}\right)\right)
\end{gathered}
$$

It is very important to note that the dg category $\operatorname{Tr}(\mathcal{C})$ is additive but not triangulated. Indeed, there are lots of morphisms in $\operatorname{Tr}(\mathcal{C})$ which do not exist in $\mathcal{C}$, so their cones do not exist as objects in $\mathcal{C}$ or in $\operatorname{Tr}(\mathcal{C})$. However, we can consider its pretriangulated hull as in section 3 , we will denote it by $\operatorname{Pretr}(\operatorname{Tr}(\mathcal{C})$ ). 
Lemma 6.25. If $\mathcal{C}$ is a dg monoidal category, then $\operatorname{Pretr}(\operatorname{Tr}(\mathcal{C})) \simeq \operatorname{Pretr}(\operatorname{Tr}(\operatorname{Pretr}(\mathcal{C})))$.

Proof. As it is a dg functor Tr sends twisted complexes to twisted complexes. Thus we have a dg functor

$$
\operatorname{Pretr}(\operatorname{Tr}(\operatorname{Pretr}(\mathcal{C}))) \hookrightarrow \operatorname{Pretr}(\operatorname{Pretr}(\operatorname{Tr}(\mathcal{C}))) \simeq \operatorname{Pretr}(\operatorname{Tr}(\mathcal{C})) .
$$

Conversely we have a dg functor $\operatorname{Pretr}(\operatorname{Tr}(\mathcal{C})) \hookrightarrow \operatorname{Pretr}(\operatorname{Tr}(\operatorname{Pretr}(\mathcal{C})))$ induced by $\mathrm{e} \hookrightarrow \operatorname{Pretr}(\mathrm{C})$ which is a quasi-inverse.

6.5. Homotopy trace-like functors. In the introduction we highlighted that the (underived) trace functor $\operatorname{Tr}_{0}: \mathcal{C} \rightarrow \operatorname{Tr}_{0}(\mathcal{C})$ for a monoidal category (or bicategory) $\mathcal{C}$ with left duals is initial among all trace-like functors from $\mathcal{C}$ to another category $\mathcal{D}$. Here we consider a dg analog of this situation.

Let $\mathcal{C}$ be a $\mathrm{dg}$ monoidal category and $\mathcal{D}$ a $\mathrm{dg}$ category. We are interested in $\mathrm{dg}$ functors $\phi: \mathcal{C} \rightarrow \mathcal{D}$ that are homotopy trace-like in the sense that for every pair of objects $X, Y$ in $\mathcal{C}$ we get natural (up to coherent homotopy) maps $\phi(X \star Y) \rightarrow \phi(Y \star X)$, possibly even isomorphisms. We define a $d g$ monoidal trace on $\mathcal{C}$ with values in $\mathcal{D}$ to be a dg functor $\phi: \mathcal{C} \rightarrow \mathcal{D}$ that factors through $\operatorname{Tr}: \mathcal{C} \rightarrow \operatorname{Tr}(\mathcal{C})$. I.e. there exists a dg functor $\phi^{\prime}: \operatorname{Tr}(\mathcal{C}) \rightarrow \mathcal{D}$ such that $\phi=\phi^{\prime} \circ \operatorname{Tr}$.

Note that a dg monoidal trace $\phi$ not only contains the data of specific morphisms $\phi^{\prime}\left(w_{X, Y}\right): \phi(X \star Y) \rightarrow \phi(Y \star X)$, but also homotopies enforcing natural compatibility relations between these morphisms. In particular, by Remark $6.22 \phi^{\prime}\left(w_{X, Y}\right)$ is natural in $Y$ but it is natural in $X$ only up to coherent higher homotopies $\phi^{\prime}\left(w\left(f_{1}\|\ldots\| f_{r} ; Y\right)\right)$.

Remark 6.26. For an underived trace-like functor $\phi=\phi^{\prime} \circ \operatorname{Tr}_{0}$ one requires (see e.g [Zhu18]) that the traciators are compatible with associators in the sense that diagrams of the following type commute:

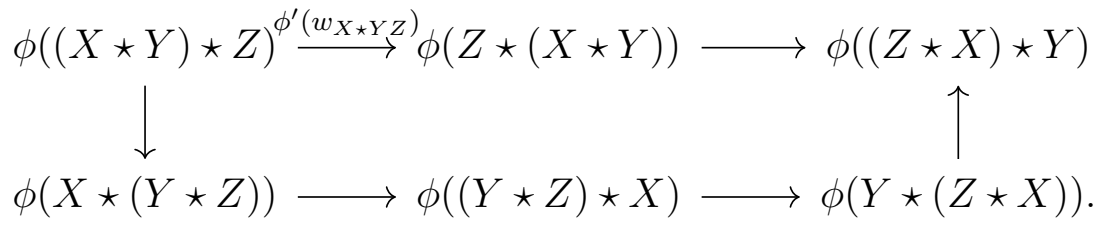

In the derived case, similar condition are required for the images of higher traciators $\phi^{\prime}\left(w\left(f_{r}\|\ldots\| f_{1} ; Y\right)\right)$.

Remark 6.27. It is likely that if $\mathcal{C}$ is has left duals then knowing $\phi^{\prime}\left(w_{X, Y}\right)$ and the naturality data $\phi^{\prime}\left(w\left(f_{1}\|\ldots\| f_{r} ; Y\right)\right)$ satisfying (18) and its analogues is enough to prove that reconstruct the functor $\phi^{\prime}$ and thus verify that $\phi$ is trace-like, but we do not prove it here.

Examples of $\mathrm{dg}$ monoidal traces are the following.

Example 6.28. Any endofunctor of $\operatorname{Tr}(\mathcal{C})$ gives rise to a $\mathrm{dg}$ monoidal trace on $\mathcal{C}$ by pre-composition with the universal trace $\mathrm{C} \rightarrow \operatorname{Tr}(\mathcal{C})$. A natural source of endofunctors of $\operatorname{Tr}(\mathcal{C})$ is the $\mathrm{dg}$ monoidal center, see Section 6.6. 
Example 6.29. Let $X$ be an object of $\operatorname{Tr}(\mathcal{C})$, then another $\mathrm{dg}$ monoidal trace is given by the universal trace $\mathcal{C} \rightarrow \operatorname{Tr}(\mathcal{C})$ composed with the representable functor $\operatorname{Hom}_{\operatorname{Tr}(\mathcal{C})}(X,-)$. The target is dgmod-End $\operatorname{Tr}_{\operatorname{Te}(}(X)$. A particularly interesting case is $X=\operatorname{Tr}(\mathbb{1})$, for which Proposition 6.20 identifies the target with $\operatorname{dgmod}-\mathrm{C}_{*}(\mathcal{C})$.

Example 6.30. If $\mathcal{C}$ is a pivotal $\mathrm{dg}$ monoidal category, then $\operatorname{Hom}_{\mathcal{C}}(\mathbb{1},-)$ is a trace-like functor with target dgmod-End $\mathcal{C}_{\mathcal{C}}(\mathbb{1})$. To see that $\operatorname{Hom}_{\mathcal{C}}(\mathbb{1},-)$ is trace-like, we compute:

$$
\operatorname{Hom}_{\mathcal{C}}(\mathbb{1}, X \star Y) \cong \operatorname{Hom}_{\mathcal{C}}\left(X^{*}, Y\right) \cong \operatorname{Hom}_{\mathcal{C}}\left(\mathbb{1}, Y \star X^{* *}\right) \cong \operatorname{Hom}_{\mathcal{C}}(\mathbb{1}, Y \star X)
$$

where the last isomorphism uses pivotality. Since $\operatorname{Hom}_{\mathfrak{C}}(\mathbb{1},-)$ is trace-like (strictly, not just up to homotopy), it factors through the $\mathrm{dg}$ monoidal trace, providing a $\mathrm{dg}$ functor

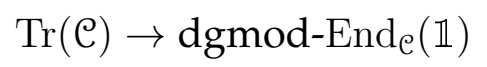

Example 6.31. A similar computation for pivotal $\mathcal{C}$ shows that if $(Z, \tau)$ is central, then the $\mathrm{dg}$ functor $\operatorname{Hom}_{\mathfrak{C}}(\mathbb{1}, Z \star-)$ is trace-like. More generally, if $\left(Z^{\prime}, \tau^{\prime}\right)$ is also central, then $\operatorname{Hom}_{\mathcal{C}}\left(Z^{\prime}, Z \star-\right)$ is again trace-like. An alternative way to construct these traces is to first factor $\operatorname{Hom}_{\mathcal{C}}(\mathbb{1},-)$ through the trace $\mathcal{C} \rightarrow \operatorname{Tr}(\mathcal{C})$ and then act by the $\mathrm{dg}$ monoidal center.

Example 6.32. The full twist FT and its powers $\mathrm{FT}^{k}$ (with suitable half-braiding data) are objects in dg Drinfeld center of the category of complexes of Soergel bimodules [EH]. By applying Example 6.31 we see that

$$
\operatorname{Hom}_{\mathrm{SBim}}\left(\mathbb{1}, \mathrm{FT}^{k} \star-\right)
$$

is a trace-like functor for all $k$. Such functors play a prominent role in the work of the first and second author, Negut and Rasmussen [GNR16, GH17].

Example 6.33. If $\mathcal{C}$ is a monoidal category, then any (ordinary) trace-like functor $\phi: \mathcal{C} \rightarrow \mathcal{D}$ lifts to a dg monoidal trace. To see this, we factor $\phi=\phi^{\prime} \circ \operatorname{Tr}_{0}$ for $\operatorname{Tr}_{0}: \mathcal{C} \rightarrow \operatorname{Tr}_{0}(\mathcal{C})$ and some functor $\phi^{\prime}: \operatorname{Tr}_{0}(\mathcal{C}) \rightarrow \mathcal{D}$. The induced dg monoidal trace is obtained by precomposing $\phi^{\prime}$ with the functor $\operatorname{Tr}(\mathcal{C}) \rightarrow \operatorname{Tr}_{0}(\mathcal{C})$ from Lemma 6.19 and the universal trace $\operatorname{Tr}: \mathcal{C} \rightarrow \operatorname{Tr}(\mathcal{C})$.

6.6. Action of the dg monoidal center on the trace. It is a basic observation that the center $Z(A)$ of an associative algebra $A$ acts on the trace $A /[A, A]$. Similarly, if we think of the trace $\operatorname{Tr}_{0}(\mathcal{C})$ of a monoidal category as $\mathcal{C}$ integrated over an annulus, the Drinfeld center $\mathcal{Z}^{0}(\mathcal{C})$ acts by "cutting open" the annulus and "gluing in" central objects or morphisms, before "resealing the annulus". In this section we describe the analogous action of the $\mathrm{dg}$ monoidal center $\mathcal{Z}^{\mathrm{dg}}(\mathcal{C})$ on the $\mathrm{dg}$ monoidal trace $\operatorname{Tr}(\mathcal{C})$.

Let $(Z, \tau)$ be an object of $\mathcal{Z}^{\mathrm{dg}}(\mathcal{C})$. The structure map $\tau$ is a map of $\mathcal{C}$, $\mathcal{C}$-bimodules $\mathrm{B}(\mathrm{C}) \rightarrow X_{12}(Z, Z)$. Using this map we have a map of $\mathcal{C}, \mathcal{C}$-bimodules given by composing:

$$
\begin{aligned}
\mathbf{B}(\mathcal{C}) \otimes_{\mathfrak{e}} X_{12}\left(X^{\prime}, X\right) & \rightarrow \mathbf{B}(\mathfrak{C}) \otimes_{\mathfrak{e}} \mathbf{B}(\mathfrak{C}) \otimes_{\mathfrak{e}} X_{12}\left(X^{\prime}, X\right) \\
& \rightarrow \mathbf{B}(\mathcal{C}) \otimes_{\mathfrak{e}} X_{12}(Z, Z) \otimes_{\mathfrak{e}} X_{12}\left(X^{\prime}, X\right) \\
& \rightarrow \mathbf{B}(\mathcal{C}) \otimes_{\mathfrak{e}} X_{12}\left(Z \star X^{\prime}, Z \star X\right) .
\end{aligned}
$$


The first of these maps is the comultiplication on the two-sided bar complex of $\mathcal{C}$, the second is an application of $\tau$, and the last is the algebra structure on the bimodule $X_{12}$ (see Remark 6.1). Applying the functor which identifies the left and right actions of $\mathcal{C}$, we obtain a map of complexes

$$
\text { Hom }_{\operatorname{Tr}(\mathcal{e})}\left(\operatorname{Tr}(X), \operatorname{Tr}\left(X^{\prime}\right)\right) \rightarrow \operatorname{Hom}_{\operatorname{Tr}(\mathcal{e})}\left(\operatorname{Tr}(Z \star X), \operatorname{Tr}\left(Z \star X^{\prime}\right)\right) .
$$

In this way, $(Z, \tau)$ determines an endofunctor $\Xi_{(Z, \tau)}: \operatorname{Tr}(\mathcal{C}) \rightarrow \operatorname{Tr}(\mathcal{C})$ defined on objects by $\operatorname{Tr}(X) \mapsto \operatorname{Tr}(Z \star X)$ and on morphisms by the above chain map. That this map respects composition of morphisms follows from the assumption that $\tau$ is a morphism of $\mathrm{dg}$ algebras and the compatibility of the shuffle product and coproduct on the two-sided bar complex.

The action on morphism complexes can be pictured as follows.
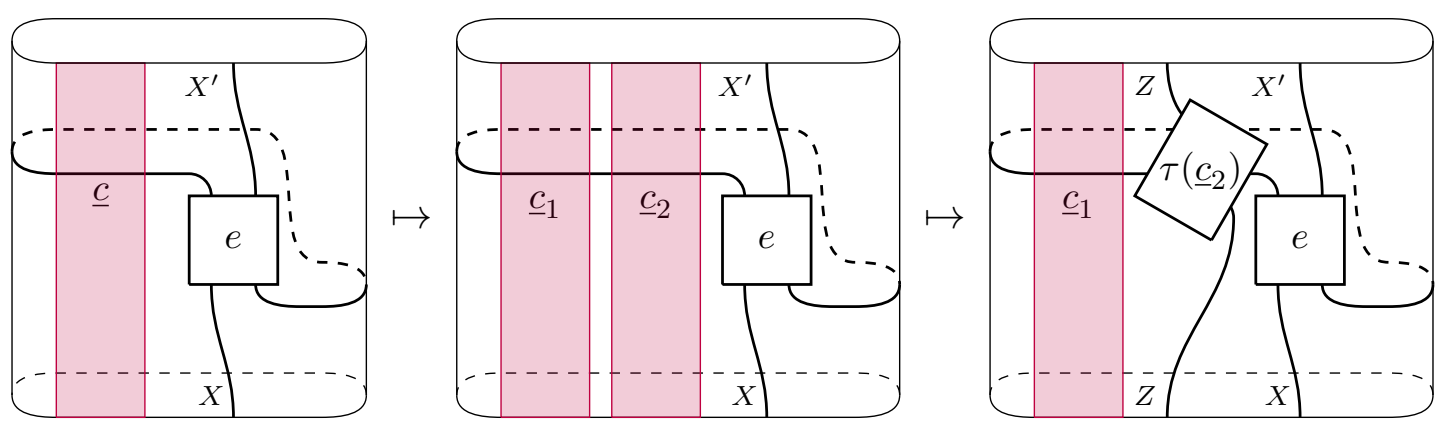

Remark 6.34. Morphisms $f:(Z, \tau) \rightarrow\left(Z^{\prime}, \tau^{\prime}\right)$ in $\mathcal{Z}^{\mathrm{dg}}(\mathcal{C})$ should give natural transformations $\Xi_{f}: \Xi_{Z, \tau} \rightarrow \Xi_{Z^{\prime}, \tau^{\prime}}$ that assemble into a monoidal functor

$$
\Xi: \mathcal{Z}^{\mathrm{dg}}(\mathcal{C}) \rightarrow \operatorname{End}(\operatorname{Tr}(\mathcal{C}))
$$

from $\mathcal{Z}^{\mathrm{dg}}(\mathcal{C})$ to the endofunctors of $\operatorname{Tr}(\mathcal{C})$. We will not pursue this in detail, see also Remark 6.4.

\section{TRACES OF THE SOERGEL CATEGORY}

7.1. Soergel bimodules. Let $W$ be a Coxeter group with simple reflections $S \subset W$, length function $\ell$, and Bruhat order $\leq$. Let $\left(V,\left\{\alpha_{s}^{\vee}\right\},\left\{\alpha_{s}\right\}\right)$ be a realization of $W$ in the sense of [EW16], over $\mathbb{C}$, which we assume to be reflection faithful and balanced unless stated otherwise. As usual, we consider the polynomial $\operatorname{ring} R=\mathbb{C}[V]:=$ $\operatorname{Sym}^{\bullet}\left(V^{*}(-2)\right)$, which is graded by declaring elements in $V^{*}$ to be of degree 2 . In particular, we have $\alpha_{s} \in R$, and these elements generate $R$ if they span $V^{*}$.

Remark 7.1. In type $A$ we have $W=S_{n}$ with simple reflections (transpositions) indexed by $i \in\{1, \ldots, n-1\}$. We will consider the realization with $V=\mathbb{C}^{n}$, on which $S_{n}$ acts by permuting standard basis vectors, and we identify $R=\mathbb{C}\left[x_{1}, \ldots, x_{n}\right]$ and $\alpha_{i}=x_{i}-x_{i+1}$ for $1 \leq i \leq n-1$.

We now describe the category of Soergel bimodules $\operatorname{SBim}(W)$ associated to $W$ and its chosen realization. For each simple reflection $s \in S$ we let

$$
B_{s}:=R \otimes_{R^{s}} R(1)
$$


where $R^{s}=\{f \in R \mid s(f)=f\}$ and (1) is the "downward" grading shift. Then the category of Soergel bimodules $\operatorname{SBim}(W)$ is the smallest full monoidal subcategory of graded $R, R$-bimodules containing the bimodules $B_{s}$ for $s \in S$ whichis closed under grading shift, isomorphism, direct sums, and direct summands. The monoidal structure on $\operatorname{SBim}(W)$ is denoted by $\star$ and tensor products of bimodules of the form $B_{s}$ are called Bott-Samelson bimodules.

In this paper we will occasionally use the diagrammatic Hecke category $\mathcal{D}_{W}$ of Elias-Williamson [EW16], whose Karoubi completion is equivalent to the category of Soergel bimodules under the assumptions taken. The diagrammatic category $\mathcal{D}_{W}$ has the advantages of being strictly monoidal and manifestly strictly pivotal (by the balanced assumption) besides being well-behaved for a larger class of realizations (which will not be relevant here).

Let $\mathrm{C}(W):=\mathrm{Ch}^{b}(\operatorname{SBim}(W))$ be the $\mathrm{dg}$ monoidal category of bounded chain complexes of Soergel bimodules for $W$. If $\beta$ is a word in the alphabet $\sigma_{s}, \sigma_{s}^{-1}$ with $i \in S$, then we have a finite complex $F(\underline{\beta}) \in \overline{\mathrm{C}}(W)$ defined by

$$
\begin{gathered}
F\left(\sigma_{s}\right):=\left(\underline{B_{s}} \rightarrow R(1)\right), \quad F\left(\sigma_{s}^{-1}\right):=\left(R(-1) \rightarrow \underline{B_{s}}\right), \\
F\left(\underline{\beta} \cdot \underline{\beta^{\prime}}\right):=F(\underline{\beta}) \star F\left(\underline{\beta^{\prime}}\right),
\end{gathered}
$$

where the maps $B_{s} \rightarrow R(1)$ and $R(-1) \rightarrow B_{s}$ are the canonical bimodule maps, defined by

$$
1 \otimes 1 \mapsto 1, \quad 1 \mapsto \frac{1}{2}\left(\alpha_{s} \otimes 1-1 \otimes \alpha_{s}\right),
$$

respectively. It is well known [Rou04, Rou06] that the complexes $F(\beta)$ satisfy the braid relations up to homotopy equivalence. We often abusively write $F(\beta)$ where $\beta \in \operatorname{Br}(W)$ is an element of the braid group associated to $W$ (when in reality the complex $F(\beta)$ depends on a choice of braid word $\underline{\beta}$ representing $\beta$ ).

Definition 7.2. For each $w \in W$, let $\Delta_{w}$ and $\nabla_{w}$ denote the Rouquier complex of the positive and negative braid lift of a chosen reduced expression of $w$.

In particular, we have $\Delta_{w} \star \nabla_{w^{-1}} \simeq \mathbb{1} \simeq \nabla_{w^{-1}} \star \Delta_{w}$ for any $w \in W$. We will sometimes write $\Delta_{w}^{-1}:=\nabla_{w^{-1}}$.

7.2. Hochschild homology of the Soergel category - linear structure. The following is well known to experts.

Proposition 7.3. The complexes $\Delta_{w}, w \in W$, generate $\mathrm{C}(W)$ with respect to cones, shifts, sums, and homotopy equivalences (and similarly for $\nabla_{w}$ ). These complexes satisfy

$$
\operatorname{Hom}_{\mathcal{C}(W)}\left(\Delta_{v}, \Delta_{w}\right) \simeq 0 \simeq \operatorname{Hom}_{\mathcal{C}(W)}\left(\nabla_{w}, \nabla_{v}\right) \quad \text { unless } \quad v \leq w .
$$

In other words, $\left\{\Delta_{v}\right\}_{v \in W}$ and $\left\{\nabla_{v}\right\}_{v \in W}$ each generate a semi-orthogonal decomposition of $\mathrm{e}(W)$ (the latter one with the opposite poset structure). 
Proof. By the main result of [LW14], one has

$$
\operatorname{Hom}_{\mathcal{C}(W)}\left(\Delta_{v}, \nabla_{w}\right) \simeq \begin{cases}R & \text { if } v=w \\ 0 & \text { otherwise }\end{cases}
$$

On the other hand, it is easy to see that $\Delta_{w}$ is filtered by $\nabla_{u}$ for $u \leq w$. Therefore if $\operatorname{Hom}_{\mathcal{C}(W)}\left(\Delta_{v}, \Delta_{w}\right) \not z 0$ then $\operatorname{Hom}_{\mathcal{C}(W)}\left(\Delta_{v}, \nabla_{u}\right) \not 40$ for some $u \leq w$, hence $v=u \leq w$. See also [GHMN19, Appendix] for more details.

Thus the following is an immediate consequence of Theorem 5.11.

Lemma 7.4. There is a deformation retract from the cyclic bar complex $\mathbf{C}(\operatorname{SBim}(W))$ to $\bigoplus_{w \in W} \mathbf{C}\left(\operatorname{End}\left(\Delta_{w}\right)\right)$.

Recall that we have chosen a realization $V$ of $W$. Consider the following complex of $\mathbb{C}$-vector spaces

$$
Z:=\left(V^{*}(-2) \rightarrow \underline{V^{*}(-2) \oplus V^{*}(-2)}\right), \quad \phi \mapsto(\phi,-\phi) .
$$

Complexes of $\mathbb{C}$-vector spaces form a symmetric monoidal (dg) category, and so we have Schur functors. The symmetric algebra of $Z$ is just

$$
\operatorname{Sym}^{\bullet}\left(V^{*}(-2) \oplus V^{*}(-2) \oplus \Sigma^{1} V^{*}(-2)\right) \cong R \otimes R \otimes \Lambda,
$$

with its differential inherited from $Z$. Here $\Lambda$ is the exterior algebra of $V^{*}$. After choosing a basis of $V$ and letting $x_{1}, \ldots, x_{n} \in V^{*}$ denote the dual basis, we can identify

$$
\operatorname{Sym}^{\bullet}(Z) \cong \mathbb{C}\left[x_{1}, \ldots, x_{n}, x_{1}^{\prime}, \ldots, x_{n}^{\prime}, \theta_{1}, \ldots, \theta_{n}\right], \quad d\left(\theta_{i}\right)=x_{i}-x_{i}^{\prime} .
$$

That is to say, $\operatorname{Sym}^{\bullet}(Z)$ is the Koszul resolution of $R$ as a bimodule over itself. We can use this resolution to compute HH. $(R)$ :

$$
\text { HH. }(R) \cong R \otimes \Lambda \text {. }
$$

We have a dg algebra map $R=\operatorname{End}_{\mathcal{C}(W)}(\mathbb{1}) \rightarrow \operatorname{End}_{\mathcal{C}(W)}\left(\Delta_{w}\right)$ sending $f \mapsto \operatorname{Id}_{\Delta_{w}} \star f$. The homotopy equivalence

$$
\operatorname{Hom}_{\mathcal{C}(W)}\left(\Delta_{w}, \Delta_{w}\right) \simeq \operatorname{Hom}_{\mathcal{C}(W)}\left(\Delta_{w}^{-1} \star \Delta_{w}, \mathbb{1}\right) \simeq \operatorname{End}_{\mathcal{C}(W)}(\mathbb{1})=R
$$

is clearly $R$-linear.

Thus, combining everything up to this point gives:

Corollary 7.5. There is a deformation retract from the cyclic bar complex $\mathbf{C}_{*}(\mathrm{e}(W))$ to its homology $R \otimes \Lambda \rtimes \mathbb{C}[W]$. Here the generators $x_{i}$ of $R$ have degree $(2,0)$, the generators $\theta_{i}$ of $\Lambda$ have degree $(2,-1)$ and $W$ has degree $(0,0)$. 
7.3. Hochschild homology of the Soergel category - algebra structure. In this section we prove Theorem 1.3. Recall that by Theorem 2.10 any deformation retract $\mathbf{C}_{*} \rightarrow H$ induces an $A_{\infty}$ structure on $H$.

Recall that the dg algebra $\mathrm{C}_{*}$ is called formal if all higher Massey products vanish (i.e. the higher $A_{\infty}$-maps on $H$ are trivial).

Theorem 7.6. We have an isomorphism of algebras

$$
\text { HH. }(\operatorname{SBim}(W)) \cong R \otimes \Lambda \rtimes W
$$

with multiplication $\mu_{2}$.

Proof. By Theorem 5.19 we have a canonical isomorphism

$$
\text { HH. }(\operatorname{SBim}(W)) \cong \mathrm{HH} \text {. }(\mathfrak{e}(W)) \text {, }
$$

and we will compute the latter. All homs in this proof are taken in $\mathcal{C}(W)$ and so the subscripts in $\operatorname{Hom}_{\mathcal{C}(W)}(-,-)$ and $\operatorname{End}_{\mathcal{C}(W)}(-)$ will be omitted. First we observe that $\mathrm{HH}$. $(\operatorname{End}(\mathbb{1})) \cong \mathrm{HH} .(R) \cong R \otimes \Lambda$ is a subalgebra of HH. $(\mathrm{C}(W))$ by definition. Second, let $\sigma_{w}$ denote the class of the identity morphism of $\operatorname{End}\left(\Delta_{w}\right)$ in HH. $(\mathrm{C}(W))$. Then $\operatorname{End}\left(\Delta_{w}\right) \simeq R$ is a bimodule over $\operatorname{End}(\mathbb{1}) \cong R$ where the right action of $R$ is standard and the left action of $R$ is twisted by $w$, and the same holds for the actions of HH. $(R)$ on $\mathrm{HH} .\left(\operatorname{End}\left(\Delta_{w}\right)\right) \simeq \mathrm{HH} .(R)$. In other words,

$$
f(x, \theta) \cdot \sigma_{w}=\sigma_{w} \cdot f\left(w^{-1}(x), w^{-1}(\theta)\right) .
$$

It remains to prove that $w \mapsto \sigma_{w}$ is a group homomorphism. For this, we construct an action of $\sigma_{w}$ on $\operatorname{End}(\mathbb{1})$ by the following composition of homotopy equivalences (where all homs are taken in $\mathrm{C}(W)$ ):

$$
\sigma_{w}: \operatorname{End}(\mathbb{1}) \simeq \operatorname{Hom}\left(\mathbb{1}, \Delta_{w} \Delta_{w}^{-1}\right) \simeq \operatorname{Hom}\left(\Delta_{w}, \Delta_{w}\right) \simeq \operatorname{Hom}\left(\mathbb{1}, \Delta_{w}^{-1} \Delta_{w}\right) \cong \operatorname{End}(\mathbb{1}) .
$$

Here the middle isomorphism follows from the fact that $\Delta_{w}$ and $\Delta_{w}^{-1}$ are biadjoint in $\mathcal{C}(W)$. One can check that this action is compatible with the shuffle multiplication in $\mathrm{HH}_{0}(\mathrm{e}(W))$ and $\sigma_{w}$ acts as a permutation $(-1)^{\ell(w)} w$ on $\operatorname{End}(\mathbb{1})$ (the signs that appear here depend on the homological shift conventions for Rouquier complexes, see [GW19, Section 4.3 and Remark 4.26] for a discussion). Since the representation of $W$ on $\operatorname{End}(\mathbb{1})$ is faithful, we get that the $\sigma_{w}$ generate a copy of $W$ inside $\mathrm{HH}_{0}(\mathfrak{C}(W))$.

Remark 7.7. The main result of [EL16] states that $\mathrm{HH}_{0}(\operatorname{SBim}(W)) \cong R \rtimes \mathbb{C}[W]$, so our theorem is a natural generalization. The methods of proof in [EL16], however, were completely different and used cellularity of $\operatorname{SBim}(W)$.

Remark 7.8. One can check that the classes $\sigma_{w}=\left[\Delta_{w}\right]$ generate a copy of $W$ in a more direct way. Indeed, for simple reflections $s$ the objects $\Delta_{s}$ satisfy braid relations in $\mathcal{C}(W)$, and hence $\left[\Delta_{s}\right]$ satisfy them too. Furthermore,

$$
\left[\Delta_{s}\right]^{2}=\left[\left(\Delta_{s}\right)^{2}\right] \simeq\left[\underline{B_{s}}(-1) \rightarrow B_{s}(1) \rightarrow R(2)\right]=[R]-\left[B_{s}\right]+\left[B_{s}\right]=[R]=1 .
$$

The middle equation follows from (13c). 
Next we would like to understand the $A_{\infty}$-structure on $\mathrm{HH} .(\operatorname{SBim}(W))$.

We can also describe the action of the Connes differential on Hochschild homology.

Proposition 7.9. The action of the Connes differential $\mathbb{B}$ on $\mathrm{HH} .(\operatorname{SBim}(W))$, pulled back to $R \otimes \Lambda \rtimes \mathbb{C}[W]$ via the isomorphism from Theorem 7.6, is given by

$$
\mathbb{B}=\sum_{i} \theta_{i} \frac{\partial}{\partial x_{i}}
$$

where $x_{i}, \theta_{i}$ are dual bases in $V, V^{*}$.

Proof. Recall the isomorphisms

$$
\text { HH. }(\operatorname{SBim}(W)) \cong \mathrm{HH}_{.}(\mathrm{C}(W)) \cong \oplus_{w \in W} \mathrm{HH} .\left(\operatorname{End}\left(T_{w}\right)\right) \cong \oplus_{w \in W} \mathrm{HH} \cdot(R) .
$$

Observe that the second isomorphism is obtained by certain retractions which commute with the action of the Connes differential $\mathbb{B}$, so it is sufficient to know the action of $\mathbb{B}$ on $\mathrm{HH}_{\text {. }}\left(\operatorname{End}\left(T_{w}\right)\right)=\mathrm{HH} .(R)$. The differential $\mathbb{B}$ on HH. $(R)$ is given by $(14)$, and it is obviously $W$-invariant.

7.4. Towards understanding formality. Recall that we have a chosen realization of the Coxeter group $W$, denoted $V$, and $R=\operatorname{Sym}\left(V^{*}(-2)\right)$ is the ring of polynomial functions on $V$, bigraded by declaring linear functions to have degree $(2,0)$. Let us choose once and for all pair of dual bases for $V$ and $V^{*}$. After this choice, we will write $R=\mathbb{C}\left[x_{1}, \ldots, x_{n}\right]$, where $x_{i}$ range over the dual basis in $V^{*}(-2)$. We will need the following auxilliary objects as well.

Let $E$ denote the bigraded algebra freely generated by elements $\xi_{i}, \xi_{i}^{*}(1 \leq i \leq n)$ of degree $\operatorname{deg}\left(\xi_{i}\right)=(2,-1), \operatorname{deg}\left(\xi_{i}^{*}\right)=(-2,1)$, modulo

$$
\left[\xi_{i}, \xi_{j}\right]=0, \quad\left[\xi_{i}^{*}, \xi_{j}^{*}\right], \quad\left[\xi_{i}^{*}, \xi_{j}\right]= \begin{cases}1 & \text { if } i=j \\ 0 & \text { else }\end{cases}
$$

Let $M=E / I$, where $I \subset E$ is the left ideal generated by the $\xi_{i}^{*}(1 \leq i \leq n)$.

We think of $\xi_{i}$ and $\xi_{i}^{*}$ as ranging over bases of $V^{*}$ and $V$, respectively; in basis independent language one would write $M=\operatorname{Sym}\left(V^{*}[1](-2)\right)$ (which is the exterior algebra on $V^{*}$ with some regrading), and $E=\operatorname{End}_{\mathbb{C}}(M)$.

Definition 7.10. We define the cube complex $K \in \mathcal{C}(W)$ as the twisted complex $K=$ $\mathbb{1} \otimes_{\mathbb{C}} M$ with twist $\alpha_{K}=\sum_{i} x_{i} \otimes \xi_{i}^{*}$. Note that this is simply the Koszul complex (an $n$-dimensional cube-like complex) associated to the action of $x_{1}, \ldots, x_{n}$ on $\mathbb{1}$.

Example 7.11. For $W=S_{2}$ and $V=\mathbb{C}$ (the reflection representation) we get

$$
K=\left(\mathbb{1} \stackrel{x_{1}-x_{2}}{\longrightarrow} \mathbb{1}\right) .
$$

Example 7.12. For $W=S_{2}$ and $V=\mathbb{C}^{2}$ (the permutation representation) we get

$$
K=\left(\mathbb{1} \stackrel{\left(x_{1}, x_{2}\right)}{\longrightarrow} \mathbb{C}^{2} \otimes \mathbb{1} \stackrel{(x 2,-x 1)}{\longrightarrow} \mathbb{1}\right)
$$


Observe that $\operatorname{Tr}(K)$ is the twisted complex $\operatorname{Tr}(\mathbb{1}) \otimes_{\mathbb{C}} M$ with twist $\alpha_{K}$. The action of $W$ on $\operatorname{Tr}(\mathbb{1})$ and on $M$ induces the action of $W$ on $\operatorname{Tr}(K)$, as the twist is $W$-invariant. The operators $x_{i}, \theta_{i} \in \operatorname{End}_{\operatorname{Tr}}(\operatorname{Tr}(\mathbb{1}))$ also act on $\operatorname{Tr}(K)$, although it is easy to see that the action of $x_{i}$ on $\operatorname{Tr}(K)$ is null-homotopic. Finally, multiplication by $\xi_{i}^{*}$ also acts on $\operatorname{Tr}(K)$.

Theorem 7.13. The endomorphism dg algebra $\operatorname{End}_{\operatorname{Tr}(e(W))}(\operatorname{Tr}(K))$ is formal and thus quasiisomorphic to its cohomology $\mathbb{C}\left[\theta_{i}, \xi_{i}^{*}\right] \rtimes \mathbb{C}[W]$.

Proof. It is easy to see that $\operatorname{End}_{\operatorname{Tr}(\mathcal{C}(W))}(\operatorname{Tr}(K))$ is quasi-isomorphic to the total complex of the bicomplex

$$
\left(\operatorname{End}_{\operatorname{Tr}(\mathcal{e}(W))}(\operatorname{Tr}(\mathbb{1})) \otimes \operatorname{End}_{\mathbb{C}}(M), D\right)
$$

where the differential $D$ consists of the internal differential $d_{\mathbf{C}}$ of $\operatorname{End}_{\operatorname{Tr}(\mathcal{C}(W))}(\operatorname{Tr}(\mathbb{1}))$, which we identity with $\mathbf{C}_{*}(\mathfrak{C}(W))$, and the "cube differential" $d_{1}$ corresponding to the commutation with the twist $\alpha_{K}$.

We can compute the homology of (21) using the following spectral sequence. First we compute the homology of $d_{\mathbf{C}}$ and obtain

$$
H^{\bullet}\left(\operatorname{End}_{\operatorname{Tr}(\mathcal{C}(W))}(\operatorname{Tr}(\mathbb{1})) \simeq \mathrm{HH}_{.}(\mathcal{C}(W)) \cong R \otimes \wedge\left(\theta_{i}\right) \rtimes \mathbb{C}[W],\right.
$$

so the $E_{1}$ page of the spectral sequence has the form

$$
E_{1}=H^{\bullet}\left(\left(\operatorname{End}_{\operatorname{Tr}(\mathcal{C}(W))}(K), d_{\mathbf{C}}\right)=R \otimes \mathbb{C}\left[\theta_{i}\right] \rtimes \mathbb{C}[W] \otimes \operatorname{End}_{\mathbb{C}}(M) .\right.
$$

Next, we compute the homology of the differential $d_{1}$. We have

$$
H^{\bullet}\left(R \otimes \operatorname{End}_{\mathbb{C}}(M),\left[\alpha_{K},-\right]\right) \simeq H^{\bullet}\left(R \otimes E,\left[\alpha_{K},-\right]\right) \simeq \wedge\left(\xi_{i}^{*}\right) .
$$

Since the twist commutes with $W$ and all $\theta_{i}$, we get the $E_{2}$ page

$$
E_{2}=H^{\cdot}\left(E_{1}, d_{1}\right)=\mathbb{C}\left[\theta_{i}, \xi_{i}^{*}\right] \rtimes \mathbb{C}[W] .
$$

Observe that here $\theta_{i}$ have bidegree $(2,-1)$, while $\xi_{i}^{*}$ have degree $(-2,1)$ and $\mathbb{C}[W]$ has bidegree $(0,0)$. Therefore the bidegrees of all homology generators in $E_{2}$ page are concentrated on the line $(2 s,-s)$.

On the other hand, any higher differentials $d_{r}$ have (total) degree $(0,1)$, so they cannot preserve this line and must vanish. We conclude that the spectral sequence collapses at the $E_{2}$ page.

Finally, we need to prove that the Massey products $\mu_{r}$ vanish for $r \geq 3$. Note that $\mu_{r}$ shift the homological degree by $(3-r)$ and do not change the $q$-degree. Since all generators in the homology of $\operatorname{End}_{\operatorname{Tr}(\mathcal{e}(W))}(K)$ are concentrated on the line $(2 s,-s)$, and $\mu_{r}$ move them out of this line for $r \geq 3$, we conclude that $\mu_{r}=0$.

Corollary 7.14. There is a functor $\operatorname{Hom}_{\operatorname{Tr}(\mathcal{e}(W))}(\operatorname{Tr}(K),-)$ from the derived horizontal trace $\operatorname{Tr}(\mathfrak{C}(W))$ to the category of $A_{\infty}$-modules over dg algebra $\operatorname{End}_{\operatorname{Tr}}(\operatorname{Tr}(K))$.

Remark 7.15. Although the dg algebra $\operatorname{End}_{\operatorname{Tr}(e(W))}(\operatorname{Tr}(K))$ is formal, it could have nontrivial $A_{\infty}$-modules. 
Example 7.16. Similarly to the above, it is easy to see that

$$
\mathrm{Hom}_{\operatorname{Tr}(\mathcal{e}(W))}(\operatorname{Tr}(K), \operatorname{Tr}(\mathbb{1})) \simeq \mathbb{C}\left[\theta_{i}\right] \rtimes \mathbb{C}[W]
$$

as a module over $\operatorname{End}_{\operatorname{Tr}}(\operatorname{Tr}(K))$. However, we do not know if it has nontrivial $A_{\infty}$ products

$$
\mu_{r}: \operatorname{End}_{\operatorname{Tr}(\mathfrak{e}(W))}(\operatorname{Tr}(K))^{\otimes r-1} \otimes \operatorname{Hom}_{\operatorname{Tr}(\mathfrak{e}(W))}(\operatorname{Tr}(K), \operatorname{Tr}(\mathbb{1})) \rightarrow \operatorname{Hom}_{\operatorname{Tr}(\mathfrak{e}(W))}(K, \operatorname{Tr}(\mathbb{1})) .
$$

Conjecture 7.17. The cyclic bar complex $\mathbf{C}_{*}(\mathfrak{C}(W)) \simeq \operatorname{End}_{\operatorname{Tr}(\mathfrak{e}(W))}(\operatorname{Tr}(\mathbb{1}))$ is formal as a dg algebra. In particular, all maps $\mu_{d}$ on the Hochschild homology $\mathrm{HH} .(\mathrm{C}(W))$ vanish for $d \geq 3$.

Remark 7.18. The previous version of this paper on arXiv contained an incomplete proof of this conjecture. The key step in the argument was using that the all deformation retract data were $R$-bilinear. This is indeed the case except possibly for the crucial homotopy retracting $\operatorname{Hom}\left(\Delta_{v}, \Delta_{w}\right) \simeq 0$ implied by Proposition 7.3. One can choose a homotopy which is $R$-linear on the left (or on the right), but it not known to us if we can choose an $R$-bilinear homotopy. Therefore, it is unclear if one can choose an $R$-bilinear retraction of the whole cyclic bar complex of $\mathrm{C}(W)$ to its homology.

We note that the Connes differential $\mathbb{B}$ induces an interesting endomorphism of $\operatorname{Tr}(K)$. Recall that $\mathbb{B}=\sum \theta_{i} \frac{\partial}{\partial x_{i}}$, and we can write the differential on $K$ as $d=$ $\sum x_{i} \xi_{i}^{*}$, so $\mathbb{B}(d)=\sum \theta_{i} \xi_{i}^{*}$. Here $\theta_{i}$ and $\xi_{i}^{*}$ correspond to two dual bases in $V^{*}$ and $V$ respectively, so $\mathbb{B}(d)$ is simply the canonical tensor in $V^{*} \otimes V$. It has bidegree $(0,0)$ and is invariant under the action of $W$.

Example 7.19. For $W=S_{2}$ and $V=\mathbb{C}$ we get the following endomorphism of $K$ :

$$
\begin{aligned}
& K=\left(\operatorname{Tr}(\mathbb{1}) \stackrel{x_{1}-x_{2}}{\longrightarrow} \operatorname{Tr}(\mathbb{1})\right) \\
& K=\left(\operatorname{Tr}(\mathbb{1}) \stackrel{x_{1}-\theta_{2}}{\longrightarrow} \operatorname{Tr}(\mathbb{1})\right) .
\end{aligned}
$$

7.5. The dg monoidal trace of the Soergel category. In this section, we let $W$ be an arbitrary finite Coxeter group and again write $\mathrm{e}(W)=\mathrm{Ch}^{b}(\operatorname{SBim}(W))$ for the $\mathrm{dg}$ monoidal category of bounded chain complexes of Soergel bimodules for $W$ with a given realisation.

Any object in $\operatorname{Tr}(\mathrm{C}(W))$ is homotopy equivalent to a twisted complex built out of finite direct sums of $\operatorname{Tr}\left(B_{w}\right)$. The same applies to any object in $\operatorname{Pretr}(\operatorname{Tr}(\mathcal{C}(W)))$. Indeed, any object $X$ in $\mathrm{C}(W)$ is a complex built out of finite direct sums of $B_{w}$, which we can write as an iterated cone. Since $\operatorname{Tr}$ is a dg functor, we can write $\operatorname{Tr}(X)$ as an iterated cone built out of $\operatorname{Tr}\left(B_{w}\right)$ which is a twisted complex. Note that $\operatorname{Tr}$ indeed sends a complex to a twisted complex in general, see Example 8.16.

Proposition 7.20. Let $X$ and $Y$ be two objects in $\mathrm{e}(W)$. Then $\operatorname{Tr}(Y) \operatorname{Tr}(\mathrm{e}(W)) \operatorname{Tr}(X)$ is homotopy equivalent to a bounded complex of free finitely generated $R$-modules. 
In particular, the homs in $\operatorname{Tr}(\mathcal{C}(W))$ are finite-dimensional in each bidegree.

Proof. Here we use the semiorthogonal decomposition from Proposition 7.3 again, and we write $\mathcal{C}=\mathcal{C}(W)$ As in Section 5.4, the hom complexes in $\operatorname{Tr}(\mathcal{C})$ retract onto semi-orthogonal hom complexes, i.e. for objects $X$ and $Y$ we have a retraction

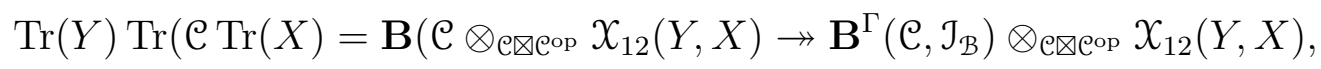

and further onto a complex which has chain groups $\bigoplus_{w_{0}}\left(\Delta_{w_{0}} Y\right) \mathcal{C}\left(X \Delta_{w_{0}}\right)$ in degree $r=0$ and for $r>0$ :

$$
\bigoplus_{r} \bigoplus_{w_{0}, \ldots, w_{r}} \Sigma^{r}\left(\Delta_{w_{0}} \mathcal{C} \Delta_{w_{1}} \otimes \cdots \otimes \Delta_{w_{r-1}} \mathcal{C} \Delta_{w_{r}} \otimes\left(\Delta_{w_{r}} Y\right) \mathcal{C}\left(X \Delta_{w_{0}}\right)\right)
$$

with $w_{0} \leq w_{1} \leq \ldots \leq w_{r}$ in Bruhat order. This still leaves us with an infinite complex since $w_{i}$ could repeat arbitrarily. However, such infinite repeats form a copy of the two-sided bar complex of $\operatorname{End}\left(\Delta_{w}\right)=R$, which can be retracted to a finite complex. Since $W$ was assumed to be finite, which implies that chains in Bruhat order are finite, performing all such retractions we arrive at a homotopy equivalent bounded complex. Freeness now follows since morphism spaces in $\mathcal{C}$ are free over $R$, see [Soe07] or [EW14, Theorem 3.6].

\section{THE SOERGEL CATEGORY IN TYPE A}

In this section we describe the derived horizontal trace of the Soergel category in type A, using the computation of its derived vertical trace in Section 7.

In Proposition 6.20 we have seen that the (derived) vertical trace $\mathrm{C}_{*}(\mathcal{C})$ of a monoidal $(\mathrm{dg})$ category $\mathcal{C}$ can be identified with the endomorphisms of $\operatorname{Tr}(\mathbb{1})$ in the (derived) horizontal trace $\operatorname{Tr}(\mathcal{C})$. That this in fact determines the entire horizontal trace for Soergel bimodules of type A is the upshot of the following theorem.

Theorem 8.1. The Karoubi completion of the triangulated hull of $\operatorname{Tr}\left(\mathrm{SBim}_{n}\right)$ is split-generated by $\operatorname{Tr}(\mathbb{1})$. We have

$$
\operatorname{Kar}^{d g}\left(\operatorname{Pretr}\left(\operatorname{Tr}\left(\operatorname{SBim}_{n}\right)\right)\right) \simeq \operatorname{Perf}\left(\mathbb{C}\left[x_{1}, \ldots, x_{n}, \theta_{1}, \ldots, \theta_{n}\right] \rtimes \mathbb{C}\left[S_{n}\right]-\text { mod }\right) .
$$

Here in the right hand side we have the category of perfect $A_{\infty}$-modules over the $A_{\infty}$-algebra

$$
\text { HH. }\left(\operatorname{SBim}_{n}\right) \cong R \otimes \Lambda \rtimes \mathbb{C}\left[S_{n}\right]=\mathbb{C}\left[x_{1}, \ldots, x_{n}, \theta_{1}, \ldots, \theta_{n}\right] \rtimes \mathbb{C}\left[S_{n}\right],
$$

where $R$ and $S_{n}$ are supported in cohomological degree zero and the variables $\theta_{i}$ have cohomological degree -1 .

The proof of this theorem will occupy the rest of this section. Here we outline the strategy of the proof. First, we use the technology of Frobenius extensions to prove that $\operatorname{Tr}\left(B_{I}\right)$ is a direct summand in a direct sum of several copies of $\operatorname{Tr}(\mathbb{1})$, where $B_{I}$ is the Soergel bimodule corresponding to the longest element in a parabolic subgroup $W_{I} \subset W$, for any subset $I \subset S$ of simple transpositions. Next, we use an explicit "annular simplification" algorithm to present $\operatorname{Tr}(B)$ as a direct summand in a direct 
sum of $\operatorname{Tr}\left(B_{I}\right)$ for any Soergel bimodule $B$. Finally, we show that any complex of Soergel bimodules is mapped by the trace functor to a summand in a finite twisted complex built out of $\operatorname{Tr}(\mathbb{1})$, thus completing the proof.

8.1. Frobenius extensions and horizontal trace. In this self-contained section we show that Soergel bimodules $B_{I}$ associated to longest elements of finite parabolic subgroups $W_{I}$ of a Coxeter group $W$ always have traces $\operatorname{Tr}_{0}\left(W_{I}\right)$ isomorphic to summands of $\operatorname{Tr}_{0}(\mathbb{1})$, after Karoubi completion. We prove this in a slightly more general setting, using the language of Frobenius extensions.

Definition 8.2. A Frobenius extension is an extension of commutative rings $\iota: A \hookrightarrow B$, such that $B$ is free and finitely generated as an $A$-module, equipped with a nondegenerate $A$-linear map $\partial: B \rightarrow A$, called the trace. Here, non-degeneracy asserts the existence of $A$-linear dual bases $\left\{x_{\alpha}\right\}$ and $\left\{y_{\alpha}\right\}$ for $B$ such that $\partial\left(x_{\alpha} y_{\beta}\right)=\delta_{\alpha, \beta}$ (the Kronecker delta).

Example 8.3. Let $(W, S)$ be a Coxeter system of finite rank as in $\S 7$ and let $R$ again denote the base ring associated with a reflection faithful balanced realization over $\mathbb{C}$. For every finite parabolic subgroup $W_{I}$, let $R^{I}$ denote the subring of $W_{I}$-invariants in $R$. Let $w_{I} \in W_{I}$ be the longest element. Then $\iota: R^{I} \rightarrow R$ is a graded Frobenius extension of rank $\left|W_{I}\right|$ with trace $\partial=\partial_{I}=\partial_{s_{1}} \cdots \partial_{s_{r}}$ where $w_{I}=s_{1} \cdots s_{r}$ is a reduced expression and

See e.g. [Wil08, Section 3].

$$
\partial_{s}(f)=\frac{f-s(f)}{\alpha_{s}} .
$$

Remark 8.4. The subsets $I$ corresponding to finite parabolic subgroups $W_{I}$ are called finitary. Note that we do not need $W$ to be finite in Example 8.3.

Example 8.5. In type A, we have $R=\mathbb{C}\left[X_{1}, \ldots, L_{n}\right]$ and $W=S_{n}$ and $R^{S_{n}} \hookrightarrow R$ is a graded Frobenius extension of rank $n$ !. An $R^{S_{n}}$-linear basis of $R$ is given by the monomials $X_{1}^{a_{1}} X_{2}^{a_{2}} \cdots X_{n-1}^{a_{n-1}}$ where $0 \leq a_{i} \leq n-i$. Then we have

$$
\partial\left(X_{1}^{a_{1}} X_{2}^{a_{2}} \cdots X_{n-1}^{a_{n-1}}\right)= \begin{cases}1 & \text { if } a_{i}=n-i \text { for } 1 \leq i \leq n, \\ 0 & \text { otherwise }\end{cases}
$$

The basis dual to the monomial basis has elements $\prod_{k=1}^{n-1}(-1)^{b_{k}} e_{b_{k}}\left(X_{n+1-k}, \ldots, X_{n}\right)$ where $b_{k}=k-a_{n-k}$ for $1 \leq k \leq n-1$.

Given a Frobenius extension $\iota: A \rightarrow B$ with trace $\partial$, we have the following maps of $B, B$-bimodules, which are best encoded diagrammatically:

- the "multiplication" $入: B \otimes_{A} B \otimes_{A} B \rightarrow B \otimes_{A} A \otimes_{A} B \cong B \otimes_{A} B$,

- the "inclusion" $1: B \rightarrow B \otimes_{A} B$ defined by $1 \mapsto \sum_{\alpha} x_{\alpha} \otimes y_{\alpha}$, and

- the "trace" १: $B \otimes_{A} B \rightarrow B$ given by $x \otimes y \mapsto x y$,

which exhibit $B \otimes_{A} B$ as a Frobenius extension of $B$. This is an instance of Jones' basic construction [Jon83]. Further we have: 
- $Y: B \otimes_{A} B \rightarrow B \otimes_{A} B \otimes_{A} B$ given by $1 \otimes 1 \mapsto 1 \otimes 1 \otimes 1$.

These satisfy the relations:

$$
\mathbf{X}=\boldsymbol{H}=\boldsymbol{Y}, \boldsymbol{\Gamma}=\boldsymbol{\lambda}=\boldsymbol{Y}=\boldsymbol{Y}=\left|, \quad \mathbf{i}=\sum_{\alpha} x_{\alpha}\right| y_{\alpha}, \quad \hat{\boldsymbol{p}}=\partial(p) \mid
$$

We also define the following shorthand notation:

$$
\cap:=\text { 人, } \cup:=Y, \quad X:=Y
$$

These morphisms satisfy the expected string-straightening and vertex rotation relations.

Definition 8.6. In the following, we will write $B_{I}:=R \otimes_{R^{I}} \otimes R$ and call this a generalised Bott-Samelson bimodule. See also [Eli16].

Lemma 8.7. With the same assumptions as in Example 8.3, we have an isomorphism

$$
\operatorname{Tr}_{0}\left(B_{I}\right) \cong \oplus_{\left|W_{I}\right|}\left(\operatorname{Tr}_{0}(\mathbb{1}), \frac{\left[\mathrm{Id}_{B_{I}}\right]}{\left|W_{I}\right|}\right)
$$

in $\operatorname{Kar} \operatorname{Tr}_{0}(\operatorname{SBim}(W))$, where we have used the identification

$$
\operatorname{End}_{\operatorname{Tr}_{0}(\operatorname{SBim}(W))}\left(\operatorname{Tr}_{0}(\mathbb{1})\right) \cong \mathrm{HH}_{0}(\operatorname{SBim}(W))
$$

to describe the idempotents appearing on the right-hand side.

Proof. The Frobenius extension provides an isomorphism $\phi: B_{I} \star B_{I} \cong \oplus_{\left|W_{I}\right|} B_{I}$, which admits a convenient diagrammatic description, c.f. [Eli16, Section 4]. To make it explicit, let $\left\{x_{\alpha}\right\}$ and $\left\{y_{\alpha}\right\}$ denote dual bases of $R$ as a free $R^{I}$-module, where $\alpha \in W_{I}$. Then $\phi=\oplus_{\alpha \in W_{I}} \phi_{\alpha}$ with

$$
\phi_{\alpha}: B_{I} \star B_{I} \rightarrow B_{I}, \quad \phi_{\alpha}\left(\left(r_{1} \star r_{2}\right) \star\left(r_{3} \star r_{4}\right)\right):=\partial\left(x_{\alpha} r_{2} r_{3}\right)\left(r_{1} \star r_{4}\right), \quad \phi_{\alpha}=\widehat{r_{\alpha}}
$$

and $\phi^{-1}=\sum_{\beta \in W_{I}} \phi_{\beta}^{-1}$ with

$$
\phi_{\beta}^{-1}: B_{I} \rightarrow B_{I} \star B_{I}, \quad \phi_{\beta}^{-1}\left(r_{1} \star r_{2}\right):=\sum_{\alpha \in W_{I}}\left(r_{1} \star 1\right) \star\left(y_{\beta} \star r_{2}\right), \quad \phi_{\alpha}=y_{\beta}
$$

Now in $\left.\operatorname{Kar}_{\operatorname{Tr}}(\operatorname{SBim}(W))\right)$ we have:

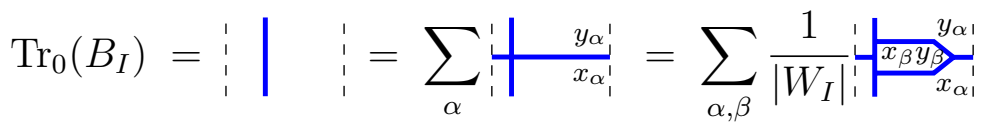

$$
\begin{aligned}
& =\sum_{\alpha, \beta} \frac{1}{\left|W_{I}\right|} \frac{\frac{1}{x_{\beta} \mid y_{\alpha \mid}}}{\mid \frac{y_{\beta}}{x_{\alpha 1}}}=\sum_{\alpha} \frac{1}{\left|W_{I}\right|} \frac{\perp y_{\alpha \mid}}{x_{\alpha_{1}}}
\end{aligned}
$$

Conversely we have:

$$
\frac{1}{\left|W_{I}\right|} \frac{1}{\left|W_{I}\right|}{ }^{x_{\alpha} y_{\beta} y_{\gamma}}=\frac{\delta_{\alpha, \beta}}{\left|W_{I}\right|}=
$$


This implies that $\frac{1}{\left|W_{I}\right|} T \quad x_{\alpha_{1}}$ and $\perp y_{\alpha} \mid$ are the components of inverse isomorphisms

$$
\operatorname{Tr}_{0}\left(B_{I}\right) \leftrightarrow \oplus_{\left|W_{I}\right|}\left(\operatorname{Tr}_{0}(\mathbb{1}), \frac{\left[\operatorname{Id}_{B_{I}}\right]}{\left|W_{I}\right|}\right)
$$

Remark 8.8. An analogous argument in the derived setting shows that

$$
\operatorname{Tr}\left(B_{I}\right) \cong \oplus_{\left|W_{I}\right|}\left(\operatorname{Tr}(\mathbb{1}), \frac{\left[\mathrm{Id}_{B_{I}}\right]}{\left|W_{I}\right|}\right)
$$

in $\operatorname{Kar} \operatorname{Tr}(\operatorname{SBim}(W)))$. The main difference is that isotopies of diagrammatic morphisms through the seam of the annulus are now only possible only up to homotopy. One thus arrives at homotopy idempotents, which split in the Karoubi envelope, as described in Section 4.

We can describe the idempotent $\frac{\left[\mathrm{Id}_{B_{I}}\right]}{\left|W_{I}\right|}$ more explicitly. Indeed, it is well known that $B_{I}$ can be presented as a twisted complex consisting of $\Delta_{w}[\ell(w)]$ for $w \in W_{I}$, so by (13c) we get

$$
\left[\operatorname{Id}_{B_{I}}\right]=\sum_{w \in W_{I}}(-1)^{\ell(w)}\left[\operatorname{Id}_{\Delta_{w}}\right]=\sum_{w \in W_{I}} w
$$

where we identified $\left[\operatorname{Id}_{\Delta_{w}}\right]$ with $(-1)^{\ell(w)} w$ in $\mathrm{HH}_{0}\left(\operatorname{SBim}_{W}\right)=\mathrm{HH}_{0}\left(\mathcal{K}^{b}\left(\operatorname{SBim}_{W}\right)\right)$ using Theorem 7.6. We get the following

Corollary 8.9. We have

$$
\operatorname{Tr}\left(B_{I}\right) \cong \oplus_{\left|W_{I}\right|}\left(\operatorname{Tr}(\mathbb{1}), \frac{1}{\left|W_{I}\right|} \sum_{w \in W_{I}} w\right) .
$$

Example 8.10. Let $W=S_{n}$ and $W_{I}=S_{k_{1}} \times \cdots \times S_{k_{r}}$ for $k_{1}+\ldots+k_{r}=n$. Then

$$
\operatorname{Tr}\left(B_{I}\right) \simeq \bigoplus_{\lambda}\left(\operatorname{Tr}(\mathbb{1}), \mathbf{e}_{\lambda}\right)^{\oplus x_{\lambda, k_{1}, \ldots, k_{r}}}
$$

where the sum is over all partitions $\lambda$ of $n, \mathbf{e}_{\lambda}$ is an idempotent in $\mathbb{C}\left[S_{n}\right]$ corresponding to the irreducible representation $V_{\lambda}$ and the graded multiplicities are given by

$$
x_{\lambda, k_{1}, \ldots, k_{r}}:=\left[k_{1}\right] ! \cdots\left[k_{r}\right] ! c_{k_{1}, \ldots, k_{r}}^{\lambda}
$$

where $c_{k_{1}, \ldots, k_{r}}^{\lambda}$ denotes the multiplicity of the Schur function $s_{\lambda}$ in the product $h_{k_{1}} \cdots h_{k_{r}}$.

8.2. Explicit annular simplification in type A. Throughout this section, we work with type $A$ Soergel bimodules corresponding to the action of $W=S_{n}$ on $V=\mathbb{C}^{n}$. We abbreviate the notation $B_{i}:=B_{s_{i}}$ for the Bott-Samelson bimodules associated to the simple reflections $s_{i} \in S_{n}$. Moreover, if $|i-j|=1$, we also consider the Soergel bimodule $B_{i j i}=B_{j i j}:=R \otimes_{R^{\left\langle s_{i}, s_{j}\right\rangle}} R$. Further, to declutter many expressions in this subsection, we will omit the $\star$ indicating the composition of bimodules.

Lemma 8.11. Any Bott-Samelson bimodule for $S_{n}$ is isomorphic to a direct summand of a direct sum of Bott-Samelson bimodules, in each of which $B_{n-1}$ appears at most once. 
Proof. This is a standard argument which uses $B_{i} B_{i} \cong B_{i}(1) \oplus B_{i}(-1), B_{i} B_{j} \cong B_{j} B_{i}$ if $|i-j|>1$, and $B_{i} B_{j} B_{i} \cong B_{i j i} \oplus B_{i}$ if $|i-j|=1$.

For each subset $I=\left\{i_{1}<\cdots<i_{k}\right\} \subset S=\{1, \cdots, n-1\}$, we define the CoxeterBott-Samelson bimodule $\underline{B}_{I}:=B_{i_{1}} B_{i_{2}} \cdots B_{i_{k}}$.

Lemma 8.12. For any Soergel bimodule $B$ the trace $\operatorname{Tr}(B)$ is isomorphic to a direct summand in the direct sum of traces of Coxeter-Bott-Samelson bimodules as defined above.

Proof. The proof is by induction on $n \geq 1$. The base case is trivial. By Lemma 8.11 we can present $B$ as a direct summand in the direct sum of objects of the form $X$ or $X B_{n-1} Y$, where $X, Y \in \operatorname{SBim}_{n-1}$. The first case is taken care of by induction. In the second case we can use Lemma 6.21 to replace $\operatorname{Tr}\left(X B_{n-1} Y\right)$ by $\operatorname{Tr}\left(Y X B_{n-1}\right)$. Now by Lemma 8.11 we can either write $Y X \subset_{\oplus} X^{\prime}$ or $Y X \subset_{\oplus} X^{\prime} B_{n-2} Y^{\prime}$ for some $X^{\prime}, Y^{\prime} \in \operatorname{SBim}_{n-2}$. Thus we either have $Y X B_{n-1} \subset_{\oplus} X^{\prime} B_{n-1}$ or

$$
\operatorname{Tr}\left(Y X B_{n-1}\right) \subset_{\oplus} \operatorname{Tr}\left(X^{\prime} B_{n-2} Y^{\prime} B_{n-1}\right) \cong \operatorname{Tr}\left(Y^{\prime} X^{\prime} B_{n-2} B_{n-1}\right)
$$

which is taken care of by induction.

For the following let $L_{n}$ denote the indecomposable Soergel bimodule corresponding to the longest element in $S_{n}$.

Lemma 8.13. In $\mathrm{SBim}_{n+1}$ we have:

$$
L_{n} B_{n} L_{n} \cong[n-1] ! L_{n+1} \oplus[n] ! L_{n}
$$

where we use quantum numbers to indicate multiple direct summands with grading shifts.

Example 8.14. For $n=2$ we have $X_{2}=B_{1}$, so $B_{1} B_{2} B_{1} \cong B_{121} \oplus B_{1}$.

Proof. Let $R=\mathbb{C}\left[X_{1}, \ldots, X_{n+1}\right]$

$$
\begin{aligned}
L_{n} B_{n} L_{n} & =R \otimes_{R^{S_{n} \times S_{1}}} R \otimes_{R^{S_{1} \times \cdots \times S_{1} \times S_{2}}} R \otimes_{R^{S_{n} \times S_{1}}} R \\
& \cong[n-1] ! R \otimes_{R^{S_{n} \times S_{1}}} R^{S_{n-1} \times S_{1} \times S_{1}} \otimes_{R^{S_{n-1} \times S_{2}}} R^{S_{n-1} \times S_{1} \times S_{1}} \otimes_{R^{S_{n} \times S_{1}}} R \\
& \cong[n-1] ! R \otimes_{R^{S_{n+1}}} R \oplus[n-1] ![n] R \otimes_{R^{S_{n} \times S_{1}}} R \\
& =[n-1] ! L_{n+1} \oplus[n] ! L_{n}
\end{aligned}
$$

Here we have used the well-known "square-switch" isomorphism of singular BottSamelson bimodules to proceed to the third line. This can, for example, be deduced from [Wu14, Lemma 11.2] - the corresponding statement for matrix factorizationsby taking homology with respect to the positive differential and forgetting the negative differential, in the terminology of loc. cit..

In the previous proof we have used the fact that $\mathbb{C}\left[X_{1}, \ldots, X_{n-1}\right]$ is a free module of (graded) rank $[n-1]$ ! of $\mathbb{C}\left[X_{1}, \ldots, X_{n-1}\right]^{S_{n-1}}$ (in fact, a Frobenius extension).

Lemma 8.15. In $\operatorname{Kar} \operatorname{Tr}\left(\operatorname{SBim}_{n}\right)$ we have that $\operatorname{Tr}\left(B_{1} \cdots B_{n-1}\right)$ is isomorphic to a summand in a direct sum of traces of Soergel bimodules corresponding to the longest elements in parabolic subgroups in $S_{n}$. 
Proof. We prove a more general statement: $\operatorname{Tr}\left(L_{k} B_{k} \cdots B_{n-1}\right)$ is isomorphic to a summand in a direct sum of Soergel bimodules corresponding to the longest elements in parabolic subgroups. Let us use induction in $k$, starting from $k=n$ where we have just $L_{n}$. For the induction step $k+1 \mapsto k \geq 1$ we have

$$
L_{k} L_{k} B_{k} \cdots B_{n-1} \cong[k] ! L_{k} B_{k} \cdots B_{n-1}
$$

and

$$
\operatorname{Tr}\left(L_{k} L_{k} B_{k} \cdots B_{n-1}\right) \cong \operatorname{Tr}\left(L_{k} B_{k} \cdots B_{n-1} L_{k}\right) \cong \operatorname{Tr}\left(L_{k} B_{k} L_{k} B_{k+1} \cdots B_{n-1}\right),
$$

and by Lemma 8.13 we have

$$
L_{k} B_{k} L_{k} B_{k+1} \cdots B_{n-1} \cong[k-1] ! L_{k+1} B_{k+1} \cdots B_{n-1} \oplus[k] ! L_{k} B_{k+1} \cdots B_{n-1} .
$$

The claimed statement now follows from the case $k=2$.

Let us give an example computation to highlight the differences compared to the annular Khovanov-Rozansky invariant of Queffelec-Rose [QR18]

Example 8.16 (The full twist on two strands). Consider the category of Soergel bimodules of type $A_{1}$. It is well-known that the Rouquier complex of the full twist on two strands can be expressed as

$$
F\left(\sigma^{2}\right) \simeq\left(\underline{B(-1)} \stackrel{x_{1}^{l}-x_{1}^{r}}{\longrightarrow} B(1) \stackrel{\text { unzip }}{\longrightarrow} R(2)\right)
$$

To compute the derived horizontal trace class of this complex we will use the following tools. First of all, just as in the underived horizontal trace, we have homotopy equivalences $\operatorname{Tr}(B) \simeq \wedge^{2}(-1) \oplus \wedge^{2}(1)$ and $\operatorname{Tr}(R) \cong \wedge^{2} \oplus S^{2}$, where $\wedge^{2}$ and $S^{2}$ indicate the isotypic components of $\operatorname{Tr}(R)$ under the natural $S_{2}$ action. Furthermore we now observe $x_{1}^{l}-x_{1}^{r}=d\left(\left\|x_{1}\right\| \operatorname{Id}_{B}\right)=d\left(w\left(x_{1} ; B\right)\right)$ as well as unzip $\circ w\left(x_{1} ; B\right)=\theta_{1} \circ$ unzip. Then the derived annular simplification proceeds as follows:

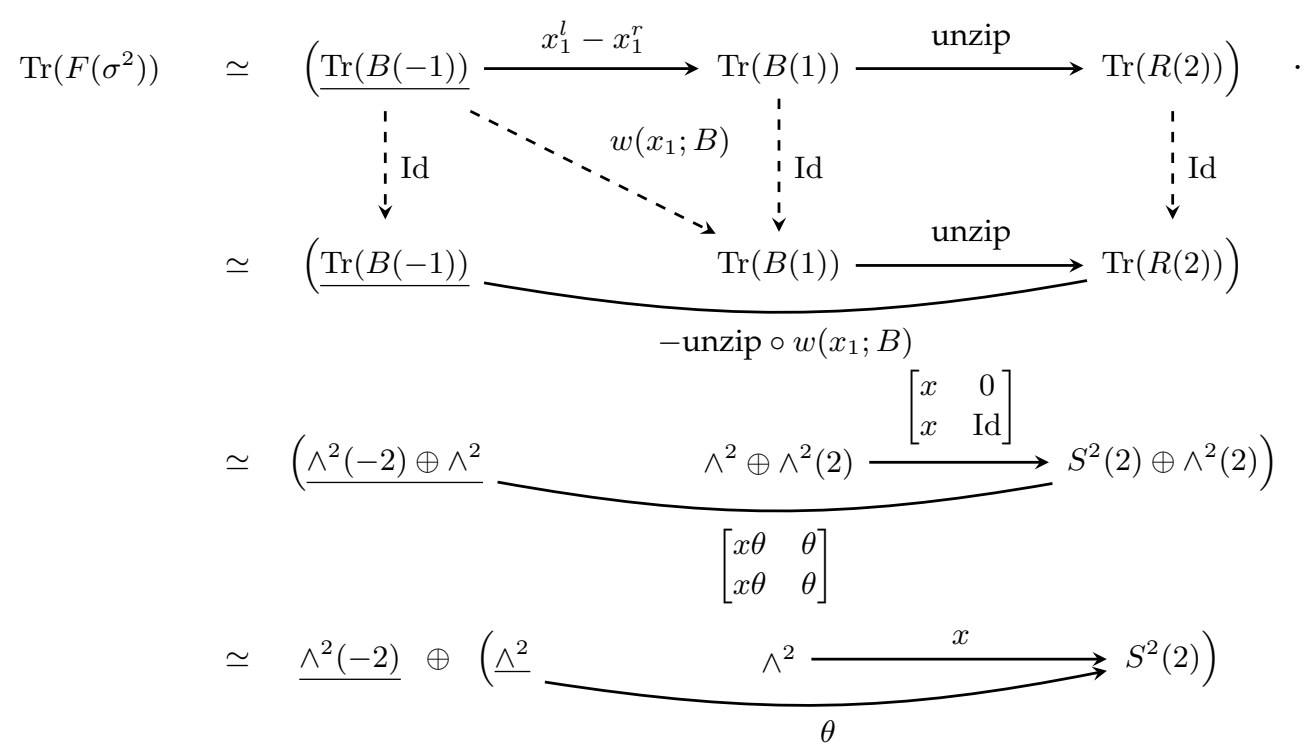


Where we abbreviate $x:=\left(x_{1}-x_{2}\right) / 2$ and $\theta:=\left(\theta_{2}-\theta_{1}\right) / 2$. Since we work over $\mathbb{C}$, we may also rescale to get $x=x_{1}-x_{2}$ and $\theta=\theta_{1}-\theta_{2}$. (Note that the second line indeed shows a twisted complex, and the dashed arrows encode a degree zero closed invertible morphism between twisted complexes.) In the underived horizontal trace the long arrow would be zero, and the complex would split into three direct summands.

8.3. Proof of Theorem 8.1. In this section we prove Theorem 8.1. First we specialize some general facts about the $A_{\infty}$-category $\operatorname{Perf}(A)$ proved in section 4.6 to the case of $A:=R \otimes \Lambda \rtimes \mathbb{C}\left[S_{n}\right]$ with maps $\mu_{d}$ induced by the retraction from the cyclic bar complex.

Recall that the generators $\theta_{i}$ of $\Lambda$ have cohomological degree -1 , so $A$ is indeed supported in nonpositive cohomological degrees. Since $A$ has zero differential, we have $\operatorname{Kar}^{d g}\langle A\rangle=\operatorname{Kar}\langle A\rangle$ where $\langle A\rangle$ is the category of free $A$ modules of finite rank. Because we work over a field of characteristic zero, $\operatorname{Kar}\langle A\rangle$ is a semisimple category with finitely many indecomposable objects labeled by irreducible representations of $S_{n}$. The category $\operatorname{Perf}(A)=\operatorname{Pretr}(\operatorname{Kar}\langle A\rangle)$ consists of twisted complexes built out of these objects. By Theorem 4.28 the category $\operatorname{Perf}(A)$ is homotopy idempotent complete.

Proof of Theorem 8.1. The quasi-equivalence between $\operatorname{Tr}\left(\operatorname{SBim}_{n}\right)$ and $\operatorname{Perf}(A)$ is constructed in two steps.

First, recall that the endomorphism algebra of $\operatorname{Tr}(\mathbb{1})$ can be identified with the cyclic bar complex $\mathbf{C}\left(\operatorname{SBim}_{n}\right)$. We have therefore a functor

$$
\operatorname{Hom}_{\operatorname{Tr}}(\operatorname{Tr}(\mathbb{1}),-): \operatorname{Tr}\left(\operatorname{SBim}_{n}\right) \rightarrow \bmod -\mathrm{C}\left(\operatorname{SBim}_{n}\right)
$$

By Lemma 8.12 we can resolve any object in $\operatorname{Tr}\left(\mathrm{SBim}_{n}\right)$ by the traces of Coxeter-BottSamelson bimodules. By Lemma 8.15 the trace of every such bimodule is equivalent to a summand in the sum of traces of indecomposable Soergel bimodules corresponding to the longest elements of parabolic subgroups. Finally, by Lemma 8.7 the trace of any such bimodule is equivalent to a summand in the direct sum of several copies of the trace of the identity bimodule.

This means that any object in the essential image of (22) is homotopy equivalent to a direct summand in a free $\mathrm{C}\left(\mathrm{SBim}_{n}\right)$-module, and (22) defines a quasi-fully faithful quasi-functor $^{2}$

$$
\operatorname{Hom}_{\operatorname{Tr}}(\operatorname{Tr}(\mathbb{1}),-): \operatorname{Tr}\left(\mathrm{SBim}_{n}\right) \rightarrow \operatorname{Kar}^{d g}\left\langle\mathbf{C}\left(\mathrm{SBim}_{n}\right)\right\rangle
$$

The corresponding functor

$$
\begin{aligned}
\operatorname{Kar}^{d g}\left(\operatorname{Pretr}\left(\operatorname{Tr}\left(\operatorname{SBim}_{n}\right)\right)\right) & \left.\rightarrow \operatorname{Kar}^{d g}\left(\operatorname{Pretr}\left(\operatorname{Kar}^{d g}\left\langle\mathbf{C}\left(\operatorname{SBim}_{n}\right)\right\rangle\right)\right)\right) \\
& =\operatorname{Kar}^{d g}\left(\operatorname{Perf}\left(\mathbf{C}\left(\operatorname{SBim}_{n}\right)\right)\right)
\end{aligned}
$$

\footnotetext{
${ }^{2}$ Note that the category of $\mathbf{C}\left(\mathrm{SBim}_{n}\right)$-modules homotopy equivalent to direct summands of free modules is quasi-equivalent to $\operatorname{Kar}^{d g}\left\langle\mathbf{C}\left(\operatorname{SBim}_{n}\right)\right\rangle$
} 
obtained by successively applying Pretr and $\operatorname{Kar}^{d g}$ on both sides is then a quasiequivalence.

For the second step, observe that by Theorem 7.6 the dg algebra $\mathrm{C}\left(\mathrm{SBim}_{n}\right)$ deformation retracts onto $A$. As in Lemma 4.30, we get quasi-equivalences $\left\langle\mathbf{C}\left(\operatorname{SBim}_{n}\right)\right\rangle \simeq\langle A\rangle$ and

$$
\operatorname{Kar}^{d g}\left(\operatorname{Perf}\left(\mathbf{C}\left(\operatorname{SBim}_{n}\right)\right)\right) \simeq \operatorname{Kar}^{d g}(\operatorname{Perf}(A)) .
$$

Since $\operatorname{Perf}(A)$ is Karoubian, $\operatorname{Kar}^{d g}(\operatorname{Perf}(A)) \simeq \operatorname{Perf}(A)$.

By combining (23) with all these quasi-equivalences, we conclude that

$$
\operatorname{Kar}^{d g}\left(\operatorname{Pretr}\left(\operatorname{Tr}\left(\operatorname{SBim}_{n}\right)\right)\right) \simeq \operatorname{Perf}(A) .
$$

Corollary 8.17. We have the following equivalence of categories:

$$
\operatorname{Kar}\left(\operatorname{Pretr}\left(\operatorname{Tr}_{0}\left(\operatorname{SBim}_{n}\right)\right)\right) \simeq \operatorname{Ch}^{b}(\operatorname{Kar}(\langle A\rangle)) \simeq \operatorname{Perf}\left(R \rtimes \mathbb{C}\left[S_{n}\right]\right) .
$$

In particular, after taking the pretriangulated hull and Karoubi completion the functor $\operatorname{Tr}\left(\operatorname{SBim}_{n}\right) \rightarrow \operatorname{Tr}_{0}\left(\operatorname{SBim}_{n}\right)$ can be identified with the forgetful functor $\epsilon: \operatorname{Perf}(A) \rightarrow \operatorname{Ch}^{b}(A)$ defined in section 4.6 .

Proof. The algebra $A$ is supported in nonpositive cohomological degrees, with $R \rtimes$ $\mathbb{C}\left[S_{n}\right]$ in cohomological degree zero. Therefore

$$
\mathrm{Ch}^{b}(\operatorname{Kar}(\langle A\rangle)) \simeq \mathrm{Ch}^{b}\left(\operatorname{Kar}\left(R \rtimes \mathbb{C}\left[S_{n}\right]\right)\right)=\operatorname{Perf}\left(R \rtimes \mathbb{C}\left[S_{n}\right]\right) .
$$

8.4. A derived annular Khovanov-Rozansky invariant. In [QR18, GW19] the (underived) traces of web categories were related to annular Khovanov-Rozansky invariants, and to the Khovanov-Rozansky homology of links in $\mathbb{R}^{3}$. In this subsection we review this construction using the category $\mathrm{SBim}_{n}$ and its trace.

Given a braid word $\beta$ on $n$ strands, let $F(\underline{\beta})$ again denote the Rouquier complex for $\beta$ as defined in Section 7.1, which we now consider as an object in $K^{b}\left(\operatorname{SBim}_{n}\right)$, the bounded homotopy category of $\operatorname{SBim}_{n}$. We have already mentioned that these complexes satisfy braid relations up to (canonical) homotopy equivalence. In fact, braid cobordisms induce natural chain maps (up to homotopy) between Rouquier complexes [EK10].

Consider the underived trace functor $\operatorname{Tr}_{0}: \operatorname{SBim}_{n} \rightarrow \operatorname{Tr}_{0}\left(\operatorname{SBim}_{n}\right)$, with the target considered as embedded in the Karoubi completion $\operatorname{Kar}\left(\operatorname{Tr}_{0}\left(\operatorname{SBim}_{n}\right)\right)$. Recall that the latter is equivalent to the category of graded projective $R \rtimes \mathbb{C}\left[S_{n}\right]$-modules. In the following definition we use the functor $\operatorname{Tr}_{0}$, extended to the bounded homotopy categories of the source and target.

Definition 8.18. The annular Khovanov-Rozansky invariant of a braid word $\beta$ on $n$ strands is defined as

$$
\operatorname{AKhR}(\underline{\hat{\beta}}):=\operatorname{Hom}_{\operatorname{Tr}_{0}}\left(\operatorname{Tr}_{0}(\mathbb{1}), \operatorname{Tr}_{0}(F(\underline{\beta}))\right) \in \operatorname{Perf}\left(R \rtimes \mathbb{C}\left[S_{n}\right]\right)
$$


By virtue of factoring through the underived trace, $\mathrm{AKhR}$ is a categorical invariant of braid conjugacy classes (a.k.a. annular links with a coherent orientation) which is natural under annular link cobordisms (preserving the coherent orientation). More precisely, AKhR is defined on $n$-strand braid words for each $n \geq 0$ separately, but these invariants fit together to give a monoidal annular link invariant, see [GW19] for details. Relatives of this notion of annular Khovanov-Rozansky invariant have previously been studied in [QR18, QRS18]. Another ahistorical aspect of our presentation here is that the interest in annular Khovanov-Rozansky invariants rose well after the construction of the triply-graded Khovanov-Rozansky homology of links in $\mathbb{R}^{3}$ [KR08, Kho07], which categorifies the HOMFLY-PT polynomial. We will comment on their relationship in the next section.

We are now ready to define a derived annular Khovanov-Rozansky invariant.

Definition 8.19. The derived annular Khovanov-Rozansky invariant is defined on closures of $n$-strand braid words $\beta$ as

$$
\operatorname{AKhR}_{\mathrm{dg}}(\underline{\hat{\beta}}):=\operatorname{Hom}_{\operatorname{Tr}}(\operatorname{Tr}(\mathbb{1}), \operatorname{Tr}(F(\underline{\beta}))) \in \operatorname{Perf}\left(R \otimes \Lambda \rtimes \mathbb{C}\left[S_{n}\right]\right)
$$

where $F(\underline{\beta})$ is the Rouquier complex of $\underline{\beta}$, Tr is the universal dg monoidal trace, and we use that $\operatorname{Hom}_{\operatorname{Tr}}(\operatorname{Tr}(\mathbb{1}),-)$ realises the equivalence from Theorem 8.1. As for the underived annular Khovanov-Rozansky invariant, this construction is functorial under braid-like annular link cobordisms between braid closures up to homotopy.

One important feature of $\operatorname{AKhR}_{\mathrm{dg}}(\underline{\hat{\beta}})$ is that the $2 \pi$ rotation of the annular link $\underline{\hat{\beta}}$ typically induces an interesting endomorphism of $\mathrm{AKhR}_{\mathrm{dg}}(\underline{\hat{\beta}})$, while it always induces the identity map on $\operatorname{KhR}(\hat{\beta})$ and its annular version.

Recall that by (20) the Connes differential $\mathbb{B}=\sum \theta_{i} \frac{\partial}{\partial x_{i}}$ defines a derivation on the algebra HH. $\left(\mathrm{SBim}_{n}\right) \cong R \otimes \Lambda \rtimes \mathbb{C}\left[S_{n}\right]$. This allows us to define an interesting endofunctor on the category of twisted $R \otimes \Lambda \rtimes \mathbb{C}\left[S_{n}\right]$-modules. Given a twisted complex $(A, d)$ we have:

$$
0=\mathbb{B}\left(d^{2}\right)=\mathbb{B}(d) d+d \mathbb{B}(d),
$$

so that $\mathbb{B}(d)$ is always a closed (degree 1 ) endomorphism of $(A, d)$.

Conjecture 8.20. The action of the rotator $w_{X}$ (defined in Remark 6.23) on twisted complexes $X$ built out of summands of $\operatorname{Tr}(\mathbb{1})$ is homotopic to $\operatorname{Id}+\mathbb{B}+$ higher order terms, where $\mathbb{B}$ is the action of Connes differential defined above.

Example 8.21. Let $f$ be an arbitrary polynomial in $R=\operatorname{End}_{\operatorname{SBim}_{n}}(\mathbb{1})$, let $X=\operatorname{Cone}(f)$. Since the rotator $w_{\mathbb{1}}: \operatorname{Tr}(\mathbb{1}) \rightarrow \operatorname{Tr}(\mathbb{1})$ is trivial, by (17) the rotator $w_{X}$ is given by the 
morphism

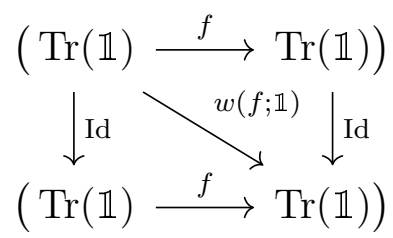

It is easy to see that $w(f ; \mathbb{1})=\|f\| \mathrm{Id}$ is homotopic to $\mathbb{B}(f)$.

Another important feature is that the derived annular Khovanov-Rozansky invariant of an annular link has an action of the derived center $\mathcal{Z}^{\mathrm{dg}}\left(\mathrm{SBim}_{n}\right)$. The second author has shown with Ben Elias that the Rouquier complex of the full twist braid $\mathrm{FT}_{n}$ (together with suitable half-braiding data) is an object of the derived Drinfeld center $[\mathrm{EH}]$. The corresponding endofunctor of the derived trace sends $\operatorname{AKhR}_{\mathrm{dg}}(\underline{\hat{\beta}})$ to $\mathrm{AKhR}_{\mathrm{dg}}\left(\widehat{F T_{n} \beta}\right)$, i.e. it cuts the thickened annulus containing the annular link $\hat{\beta}$ and re-glues it after a $2 \pi$ twist to create $\widehat{F T_{n} \beta}$.

This operation suggests that $\mathrm{AKhR}_{\mathrm{dg}}$ should be considered as an invariant of (coherently oriented) links in $S^{1} \times D^{2}$, which can be computed by choosing an $I$ bundle structure on $S^{1} \times D^{2}$, but which comes with the data necessary to change I-bundle structure.

\subsection{Triply graded homology and the Hochschild cohomology of Soergel bimod-} ules. In this section we focus on the Hochschild cohomology of individual Soergel bimodules $M$ in $\operatorname{SBim}(W)$ (we will soon specialise to $\operatorname{SBim}_{n}$ ), not on the Hochschild (co)homology of the category $\mathrm{SBim}_{n}$.

We define $\mathrm{HH}^{i}(M):=\operatorname{Ext}^{i}(R, M)$ where the Ext groups are computed in the category of $R, R$-bimodules. In particular, $\mathrm{HH}^{0}(M)=\operatorname{Hom}(R, M)$.

Given a complex $C=\left[\ldots \rightarrow M_{j} \rightarrow M_{j+1} \rightarrow \ldots\right]$ of Soergel bimodules, we define a complex of graded vector spaces

$$
\mathrm{HH}^{i}(C):=\left[\ldots \rightarrow \mathrm{HH}^{i}\left(M_{j}\right) \rightarrow \mathrm{HH}^{i}\left(M_{j+1}\right) \rightarrow \ldots\right] .
$$

Definition 8.22. Given a braid word $\beta$ on $n$ strands, the triply-graded KhovanovRozansky homology of the braid closure $\overline{\hat{\beta}}$ is defined as

$$
\operatorname{KhR}(\hat{\beta}):=H^{\bullet}\left(\oplus_{i} H^{i}(F(\underline{\beta}))\right)
$$

where $F(\underline{\beta})$ is the associated Rouquier complex of Soergel bimodules in $\mathrm{SBim}_{n}$ defined in Section 7.1. The vector space $\operatorname{KhR}(\hat{\beta})$ is triply-graded by $q$-degree, cohomological degree in the complex $F(\underline{\beta})$, and Hochschild degree $i$.

As defined, KhR is a braid conjugacy invariant, i.e. an invariant of links in a thickened annulus that are obtained from braid closures. However, after an overall grading shift, KhR becomes invariant under the second Markov move, and thus an invariant of links in $\mathbb{R}^{3}$. Since braid cobordisms induce natural chain maps (up to 
homotopy) between Rouquier complexes, one also has induced morphisms between the Khovanov-Rozansky homologies. If we also take into account the morphisms induced by braid conjugation on the level of Hochschild homology, this can be summarised by saying that KhR is functorial under braidlike annular link cobordisms between braid closures.

Next we explain how the triply-graded Khovanov-Rozansky homology can be recovered from the annular Khovanov-Rozansky invariant (derived or underived). For an alternative but related approach see [QRS18].

It is well-known that for arbitrary Soergel bimodules $M$ and $N$ there is a natural isomorphism

$$
\mathrm{HH}^{i}(M \otimes N) \cong \mathrm{HH}^{i}(N \otimes M)
$$

of $q$-graded vector spaces. In other words, $\mathrm{HH}^{i}$ is a trace-like linear functor and hence factors through $\operatorname{Tr}_{0}$. Moreover, in type A Rasmussen proved [Ras15, Proposition 4.6] that $\mathrm{HH}^{i}(M)$ is free over $R$. (More generally this is known for all Weyl groups, see Webster-Williamson [WW11]).

Lemma 8.23. For a Soergel bimodule $M$ in $\operatorname{SBim}_{n}$ we have

$$
\mathrm{HH}^{i}(M) \cong \operatorname{Hom}_{\operatorname{Tr}_{0}}\left(\mathbf{e}_{i, 1^{n-i}} \operatorname{Tr}_{0}(\mathbb{1}), \operatorname{Tr}_{0}(M)\right) \oplus \operatorname{Hom}_{\operatorname{Tr}_{0}}\left(\mathbf{e}_{i-1,1^{n-i+1}} \operatorname{Tr}_{0}(\mathbb{1}), \operatorname{Tr}_{0}(M)\right) .
$$

where $\mathbf{e}_{i, 1^{n-i}}$ is the idempotent in $\mathbb{C}\left[S_{n}\right]$ corresponding to the hook partition $\left(i, 1^{n-i}\right)$. In particular,

$$
\operatorname{Hom}(\mathbb{1}, M) \cong \operatorname{HH}^{0}(M) \cong \operatorname{Hom}_{\operatorname{Tr}_{0}}\left(\mathbf{e}_{-} \operatorname{Tr}_{0}(\mathbb{1}), \operatorname{Tr}_{0}(M)\right)
$$

where $\mathbf{e}_{-}=\mathbf{e}_{1^{n}}$ is the antisymmetrizer in $\mathbb{C}\left[S_{n}\right]$. The isomorphisms above are natural in $M$.

Proof. By (25) the functor $\mathrm{HH}^{i}$ is a trace-like functor from $\mathrm{SBim}_{n}$ to graded vector spaces, so as discussed in Section 6.5 it factors through $\operatorname{Tr}\left(\operatorname{SBim}_{n}\right)$ and defines a functor $\mathrm{HH}^{i}: \operatorname{Tr}\left(\mathrm{SBim}_{n}\right) \rightarrow$ Vect. By Theorem 8.1 we have that $\operatorname{Tr}(M)$ is isomorphic to the direct sum of direct summands of $\operatorname{Tr}(\mathbb{1})$, so it is sufficient to check it for $M=\operatorname{Tr}(\mathbb{1})$. Now

$$
\mathrm{HH}^{i}(R) \cong R \otimes \wedge^{i}\left(\mathbb{C}^{n}\right) \cong R \otimes\left(V_{i, 1^{n-i}} \oplus V_{i-1,1^{n-i+1}}\right) .
$$

while

$$
\operatorname{Hom}_{\operatorname{Tr}_{0}}\left(\operatorname{Tr}_{0}(\mathbb{1}), \operatorname{Tr}_{0}(\mathbb{1})\right) \mathbf{e}_{\lambda} \cong\left(R \rtimes \mathbb{C}\left[S_{n}\right]\right) \mathbf{e}_{\lambda} \cong R \otimes V_{\lambda}
$$

for any irreducible representation $V_{\lambda}$ of $S_{n}$.

Theorem 8.24. For any complex $C$ of Soergel bimodules one has the following isomorphism of complexes of graded vector spaces:

$$
\mathrm{HH}^{i}(C) \cong \operatorname{Hom}_{\operatorname{Tr}_{0}}\left(\mathbf{e}_{i, 1^{n-i}} \operatorname{Tr}_{0}(\mathbb{1}), \operatorname{Tr}_{0}(M)\right) \oplus \operatorname{Hom}_{\operatorname{Tr}_{0}}\left(\mathbf{e}_{i-1,1^{n-i+1}} \operatorname{Tr}_{0}(\mathbb{1}), \operatorname{Tr}_{0}(C)\right) .
$$

Proof. The functor $\operatorname{Tr}_{0}$ sends $C$ to

$$
\operatorname{Tr}_{0}(C)=\left[\ldots \rightarrow \operatorname{Tr}_{0}\left(M_{j}\right) \rightarrow \operatorname{Tr}_{0}\left(M_{j+1}\right) \rightarrow \ldots\right]
$$

with no higher differentials. Now the statement follows from Lemma 8.23. 
Theorem 8.24 implies that Khovanov-Rozansky homology can be computed by first applying the functor $\operatorname{Tr}_{0}$ to $F(\beta)$, then running the annular simplification from Theorem 8.1.

Proposition 8.25. For a braid word $\underline{\beta}$ on $n$ strands we have:

$$
\operatorname{KhR}(\hat{\beta}) \cong \oplus_{i} \operatorname{Hom}_{\operatorname{Tr}_{0}}\left(\left(\mathbf{e}_{i, 1^{n-i}}+\mathbf{e}_{i-1,1^{n-i+1}}\right) \operatorname{Tr}_{0}(\mathbb{1}), \operatorname{Tr}_{0}(F(\underline{\beta}))\right)
$$

Remark 8.26. In [GW19] the first and third authors defined the evaluation functor $\operatorname{Tr}_{0}\left(\operatorname{SBim}_{n}\right) \rightarrow$ Vect which sends $\operatorname{Tr}_{0}(\mathbb{1})$ to $R$ as a module over $R \rtimes \mathbb{C}\left[S_{n}\right]$. This is equivalent to the above since

$$
\operatorname{Hom}_{\operatorname{Tr}_{0}}\left(\mathbf{e}_{-} \operatorname{Tr}_{0}(\mathbb{1}), \operatorname{Tr}_{0}(\mathbb{1})\right)=\left(R \rtimes \mathbb{C}\left[S_{n}\right]\right) \mathbf{e}_{-}=R .
$$

Similarly, higher Khovanov-Rozansky homology $\mathrm{HH}^{i}$ can be computed by evaluating $\operatorname{Tr}(\mathbb{1})$ to $\mathrm{HH}^{i}(R)=R \otimes \wedge^{i}\left(\mathbb{C}^{n}\right)$ as a module over $R \rtimes \mathbb{C}\left[S_{n}\right]$.

\section{REFERENCES}

[AF19] David Ayala and John Francis. A factorization homology primer. In Handbook of Homotopy Theory. Chapman and Hall/CRC, 2019. arXiv:2019arXiv190310961A.

[BV08] Alexander Beilinson and Vadim Vologodsky. A DG guide to Voevodsky's motives. Geom. Funct. Anal., 17(6):1709-1787, 2008. MR2399083 DOI:10.1007/s00039-007-0644-5 arXiv:math/0604004.

[BHLv17] Anna Beliakova, Kazuo Habiro, Aaron D. Lauda, and Marko Živković. Trace decategorification of categorified quantum $\mathfrak{s l}_{2}$. Math. Ann., 367(1-2):397-440, 2017. MR3606445 DOI:10.1007/s00208-016-1389-y arXiv:1404.1806.

[BPW19] Anna Beliakova, Krzysztof K. Putyra, and Stephan M. Wehrli. Quantum link homology via trace functor I. Invent. Math., 215(2):383-492, 2019. MR3910068 DOI:10.1007/s00222018-0830-0 arXiv:1605.03523.

[BZFN10] David Ben-Zvi, John Francis, and David Nadler. Integral transforms and Drinfeld centers in derived algebraic geometry. J. Amer. Math. Soc., 23(4):909-966, 2010. MR2669705 DOI:10.1090/S0894-0347-10-00669-7 arXiv:0805.0157.

[BN09] David Ben-Zvi and David Nadler. The Character Theory of a Complex Group, 2009. arXiv:0904.1247.

[BGHT99] François Bergeron, Adriano M Garsia, Mark Haiman, and Glenn Tesler. Identities and positivity conjectures for some remarkable operators in the theory of symmetric functions. Methods and Applications of Analysis, 6(3):363-420, 1999.

[BFO09] Roman Bezrukavnikov, Michael Finkelberg, and Victor Ostrik. On tensor categories attached to cells in affine Weyl groups. III. Israel J. Math., 170:207-234, 2009. MR2506324 DOI:10.1007/s11856-009-0026-9 arXiv:math/0605628.

[BT] Roman Bezrukavnikov, Kostiantyn Tolmachov, in preparation.

[BN93] Marcel Bökstedt and Amnon Neeman. Homotopy limits in triangulated categories. Compositio Math., 86(2):209-234, 1993. MR1214458.

[BKR01] Tom Bridgeland, Alastair King, and Miles Reid. The McKay correspondence as an equivalence of derived categories. J. Amer. Math. Soc., 14(3):535-554, 2001. MR1824990 DOI:10.1090/S0894-0347-01-00368-X arXiv:math/9908027.

[EH] Benjamin Elias and Matthew Hogancamp. In preparation.

[EK95] David E. Evans and Yasuyuki Kawahigashi. On Ocneanu's theory of asymptotic inclusions for subfactors, topological quantum field theories and quantum doubles. Internat. J. Math., 6(2):205-228, 1995. MR1316301 DOI:10.1142/S0129167X95000468. 
[EK10] Ben Elias and Dan Krasner. Rouquier complexes are functorial over braid cobordisms. Homology Homotopy Appl., 12(2):109-146, 2010. MR2721032 euclid.hha/1296223879 arXiv:0906.4761.

[EL16] Ben Elias and Aaron D. Lauda. Trace decategorification of the Hecke category. J. Algebra, 449:615-634, 2016. MR3448186 DOI:10.1016/j.jalgebra.2015.11.028 arXiv:1504.05267.

[Eli16] Ben Elias. Thicker Soergel calculus in type A. Proc. Lond. Math. Soc. (3), 112(5):924-978, 2016. MR3502025 DOI:10.1112/plms/pdw012 arXiv:1009.2120.

[EW14] Ben Elias and Geordie Williamson. The Hodge theory of Soergel bimodules. Ann. of Math. (2), 180(3):1089-1136, 2014. MR3245013 DOI:10.4007/annals.2014.180.3.6 arXiv:1212.0791.

[EW16] Ben Elias and Geordie Williamson. Soergel calculus. Represent. Theory, 20:295-374, 2016. MR3555156 DOI:10.1090/ert/481 arXiv:1309.0865.

[EZ53] Samuel Eilenberg and J. A. Zilber. On products of complexes. Amer. J. Math., 75:200-204, 1953. MR52767 DOI:10.2307/2372629.

[GH17] Eugene Gorsky and Matthew Hogancamp. Hilbert schemes and $y$-ification of khovanovrozansky homology, 2017. arXiv:1712.03938.

[GHMN19] Eugene Gorsky, Matthew Hogancamp, Anton Mellit, and Keita Nakagane. Serre duality for Khovanov-Rozansky homology. 2019. arXiv:1902.08281.

[GNR16] Eugene Gorsky, Andrei Neguţ, and Jacob Rasmussen. Flag Hilbert schemes, colored projectors and Khovanov-Rozansky homology, 2016. arXiv:1608.07308.

[GW19] Eugene Gorsky and Paul Wedrich. Evaluations of annular Khovanov-Rozansky homology, 2019. arXiv:1904.04481.

[Hai01] Mark Haiman. Hilbert schemes, polygraphs and the Macdonald positivity conjecture. J. Amer. Math. Soc., 14(4):941-1006, 2001. MR1839919 DOI:10.1090/S0894-0347-01-003733 arXiv:math/0010246.

[HKR62] G. Hochschild, Bertram Kostant, and Alex Rosenberg. Differential forms on regular affine algebras. Trans. Amer. Math. Soc., 102:383-408, 1962. MR142598 DOI:10.2307/1993614.

[Hog17] Matthew Hogancamp. Idempotents in triangulated monoidal categories, 2017. arXiv:1703.01001.

[HPT16] André Henriques, David Penneys, and James Tener. Categorified trace for module tensor categories over braided tensor categories. Doc. Math., 21:1089-1149, 2016. MR3578212 arXiv:1509.02937.

[HSS17] Marc Hoyois, Sarah Scherotzke, and Nicolò Sibilla. Higher traces, noncommutative motives, and the categorified Chern character. Adv. Math., 309:97-154, 2017. MR3607274 DOI:10.1016/j.aim.2017.01.008 arXiv:1511.03589.

[Jon83] Vaughan F. R. Jones. Index for subfactors. Invent. Math., 72(1):1-25, 1983. MR696688 DOI:10.1007/BF01389127.

[Kad80] Tornike V Kadeishvili. On the homology theory of fibre spaces. Russian Mathematical Surveys, 35(3):231, 1980. MR580645.

[Kel01] Bernhard Keller. Introduction to $A$-infinity algebras and modules. Homology Homotopy Appl., 3(1):1-35, 2001. MR1854636 DOI:10.4310/hha.2001.v3.n1.a1 arXiv:math/9910179.

[Kel06] Bernhard Keller. On differential graded categories. In International Congress of Mathematicians. Vol. II, pages 151-190. Eur. Math. Soc., Zürich, 2006. MR2275593.

[Kho07] Mikhail Khovanov. Triply-graded link homology and Hochschild homology of Soergel bimodules. Internat. J. Math., 18(8):869-885, 2007. MR2339573 DOI:10.1142/S0129167X07004400 arXiv:math/0510265. 
[KR08] Mikhail Khovanov and Lev Rozansky. Matrix factorizations and link homology. II. Geom. Topol., 12(3):1387-1425, 2008. MR2421131 arXiv:math.QA/0505056 DOI:10.2140/gt.2008.12.1387.

[Kuz09] Alexander Kuznetsov. Hochschild homology and semiorthogonal decompositions, 2009. arXiv:0904.4330.

[LH03] Kenji Lefèvre-Hasegawa. Sur les A-infini catégories. PhD thesis, U. Paris 7, 2003. arXiv:math/0310337.

[Li] Penghui Li. Derived categories of character sheaves. arXiv:1803.04289

[LW14] Nicolas Libedinsky and Geordie Williamson. Standard objects in 2-braid groups. Proc. Lond. Math. Soc. (3), 109(5):1264-1280, 2014. MR3283617 DOI:10.1112/plms/pdu022 arXiv:1205.4206.

[LQ84] Jean-Louis Loday and Daniel Quillen. Cyclic homology and the Lie algebra homology of matrices. Comment. Math. Helv., 59(4):569-591, 1984. MR780077 DOI:10.1007/BF02566367.

[Lus84] G. Lusztig. Intersection cohomology complexes on a reductive group. Invent. Math., 75(2):205-272, 1984. MR732546 DOI:10.1007/BF01388564.

[MV10] Marco Mackaay and Pedro Vaz. The diagrammatic Soergel category and $\operatorname{sl}(N)$-foams, for $N \geq$ 4. Int. J. Math. Math. Sci., pages Art. ID 468968, 20, 2010. MR2671770 arXiv:0911.2485.

[Mer99] S. A. Merkulov. Strong homotopy algebras of a Kähler manifold. Internat. Math. Res. Notices, (3):153-164, 1999. MR1672242 DOI:10.1155/S1073792899000070 arXiv:math/9809172.

[MWW19] Scott Morrison, Kevin Walker, and Paul Wedrich. Invariants of 4-manifolds from Khovanov-Rozansky link homology, 2019. arXiv:1907.12194.

[Ocn94] Adrian Ocneanu. Chirality for operator algebras. In Subfactors (Kyuzeso, 1993), pages 39-63. World Sci. Publ., River Edge, NJ, 1994. MR1317353.

[PVdB19] Alexander Polishchuk and Michel Van den Bergh. Semiorthogonal decompositions of the categories of equivariant coherent sheaves for some reflection groups. J. Eur. Math. Soc. (JEMS), 21(9):2653-2749, 2019. MR3985610 DOI:10.4171/JEMS/890 arXiv:1503.04160.

[PS13] Kate Ponto and Michael Shulman. Shadows and traces in bicategories. J. Homotopy Relat. Struct., 8(2):151-200, 2013. MR3095324 DOI:10.1007/s40062-012-0017-0 arXiv:0910.1306.

[QR16] Hoel Queffelec and David E. V. Rose. The $\mathfrak{s l}_{n}$ foam 2-category: a combinatorial formulation of Khovanov-Rozansky homology via categorical skew Howe duality. Adv. Math., 302:1251-1339, 2016. MR3545951 DOI:10.1016/j.aim.2016.07.027 arXiv:1405.5920.

[QR18] Hoel Queffelec and David E. V. Rose. Sutured annular Khovanov-Rozansky homology. Trans. Amer. Math. Soc., 370(2):1285-1319, 2018. MR3729501 DOI:10.1090/tran/7117 arXiv:1506.08188.

[QRS18] Hoel Queffelec, David E. V. Rose, and Antonio Sartori. Annular evaluation and link homology, 2018. arXiv:1802.04131.

[QW18] Hoel Queffelec and Paul Wedrich. Khovanov homology and categorification of skein modules, 2018. arXiv:1806.03416.

[Ras15] Jacob Rasmussen. Some differentials on Khovanov-Rozansky homology. Geom. Topol., 19(6):3031-3104, 2015. MR3447099 DOI:10.2140/gt.2015.19.3031 arXiv:math/0607544.

[Rid13] Laura Rider. Formality for the nilpotent cone and a derived Springer correspondence. Adv. Math., 235:208-236, 2013. MR3010057 DOI:10.1016/j.aim.2012.12.001 arXiv:1206.4343.

[RR16] Laura Rider and Amber Russell. Perverse sheaves on the nilpotent cone and Lusztig's generalized Springer correspondence. In Lie algebras, Lie superalgebras, vertex algebras and 
related topics, volume 92 of Proc. Sympos. Pure Math., pages 273-292. Amer. Math. Soc., Providence, RI, 2016. MR3644235 arXiv:1409.7132.

[RW16] David E.V. Rose and Paul Wedrich. Deformations of colored $\mathfrak{s l}(N)$ link homologies via foams. Geom. Topol., 20(6):3431-3517, 2016. MR3590355 DOI:10.2140/gt.2016.20.3431 arXiv:1501.02567.

[Rou04] Raphaël Rouquier. Categorification of the braid groups, 2004. arXiv:0409593.

[Rou06] Raphaël Rouquier. Categorification of $\mathfrak{s l}_{2}$ and braid groups. In Trends in representation theory of algebras and related topics, volume 406 of Contemp. Math., pages 137-167. Amer. Math. Soc., Providence, RI, 2006. MR2258045 DOI:10.1090/conm/406/07657.

[Sei08] Paul Seidel. Fukaya categories and Picard-Lefschetz theory. Zurich Lectures in Advanced Mathematics. European Mathematical Society (EMS), Zürich, 2008. MR2441780 DOI:10.4171/063.

[Soe90] Wolfgang Soergel. Kategorie $\mathcal{O}$, perverse Garben und Moduln über den Koinvarianten zur Weylgruppe. J. Amer. Math. Soc., 3(2):421-445, 1990. MR1029692 DOI:10.2307/1990960.

[Soe92] Wolfgang Soergel. The combinatorics of Harish-Chandra bimodules. J. Reine Angew. Math., 429:49-74, 1992. MR1173115 DOI:10.1515/crll.1992.429.49.

[Soe07] Wolfgang Soergel. Kazhdan-Lusztig-Polynome und unzerlegbare Bimoduln über Polynomringen. J. Inst. Math. Jussieu, 6(3):501-525, 2007. MR2329762 DOI:10.1017/S1474748007000023.

[Tab05] Goncalo Tabuada. Une structure de catégorie de modèles de Quillen sur la catégorie des dg-catégories. C. R. Math. Acad. Sci. Paris, 340(1):15-19, 2005. MR2112034 DOI:10.1016/j.crma.2004.11.007 arXiv:math/0407338.

[Toë07] Bertrand Toën. The homotopy theory of $d g$-categories and derived Morita theory. Invent. Math., 167(3):615-667, 2007. MR2276263 DOI:10.1007/s00222-006-0025-y arXiv:math/0408337.

[Wal] Kevin Walker. Topological quantum field theories. Available at http: / / canyon23. net / math/.

[WW11] Ben Webster and Geordie Williamson. The geometry of Markov traces. Duke Math. J., 160(2):401-419, 2011. MR2852120 DOI:10.1215/00127094-1444268 arXiv:0911.4494.

[Wed19] Paul Wedrich. Exponential growth of colored HOMFLY-PT homology. Adv. Math., 353:471525, 2019. MR3982970 DOI:10.1016/j.aim.2019.06.023 arXiv:1602.02769.

[Wil08] Geordie Williamson. Singular Soergel bimodules, 2008. Dissertation, Freiburg, http: //people.mpim-bonn.mpg.de/geordie/GW-thesis.pdf.

[Wu14] Hao Wu. A colored $\mathfrak{s l}(N)$ homology for links in $S^{3}$. Dissertationes Math., 499:217, 2014. MR3234803 DOI:10.4064/dm499-0-1 arXiv:0907.0695.

[Zhu18] Xinwen Zhu. Geometric Satake, categorical traces, and arithmetic of Shimura varieties. In Current developments in mathematics 2016, pages 145-206. Int. Press, Somerville, MA, 2018. MR3837875 arXiv:1810.07375.

E.G.: Department of Mathematics, University of CAlifornia at Davis, One Shields AVENUE, DAVIS CA 95616

E. G.: Moscow State University, Faculty of Mathematics and Mechanics, GSP-1, Moscow 119991, RUSSIA

E-mail address: egorskiy@math.ucdavis.edu

M.H.: Department of Mathematics, Northeastern University, 360 Huntington Ave, BOSTON, MA 02115

E-mail address: m. hogancamp@northeastern.edu 
P.W.: Max Planck Institute for Mathematics, Vivatsgasse 7, 53111 Bonn, Germany

P.W.: Mathematical Institute, University of BonN, Endenicher Allee 60, 53115 BonN, GERMANY

P.W.: Mathematical Sciences Research Institute, 17 Gauss Way, Berkeley, CA 94720. PAUL.WEDRICH.AT

E-mail address: $\mathrm{p}$.wedrich@gmail.com 